\title{
Photoinduced intercomponent processes in selectively addressable bichromophoric dyads made of linearly-arranged Ru(II) terpyridine and expanded pyridinium components
}

Fausto Puntoriero, Antonino Arrigo, Antonio Santoro, Giuseppina La Ganga, Fabien Tuyèras, Sebastiano Campagna, Gregory Dupeyre, and Philippe P. Lainé.

\section{SUPPORTING INFORMATION}

\section{Table of Contents}

Page(s)

-Materials and methods

-Absorption spectra, luminescence properties and pump-probe transient absorption spectroscopy S1

-Electrochemistry

-Additional Figures for the photophysical properties:

Figure S1

Figure S2

-Syntheses and Characterization.

-General Experimental Details. Characterizations.

-Syntheses. Chemicals.

-General reaction schemes

-Preparation of dyads 1, 2, 3 and related precursors

-References.

$-{ }^{1} \mathrm{H}$ NMR spectra of precursors and target molecules

$-{ }^{13} \mathrm{C}$ NMR spectra $(126 \mathrm{MHz})$ of precursors and target molecules. $\mathrm{S} 25-\mathrm{S} 38$

-Mass spectra of precursors and target molecules. $\mathrm{S} 39-\mathrm{S} 46$ 


\section{Materials and methods}

\section{Absorption spectra, luminescence properties and pump-probe transient absorption spectroscopy}

UV/Vis absorption spectra were taken on a Jasco V-560 spectrophotometer. For steady-state luminescence measurements, a Jobin Yvon-Spex Fluoromax $\mathrm{P}$ spectrofluorimeter was used, equipped with a Hamamatsu R3896 photomultiplier. The spectra were corrected for photomultiplier response using a program purchased with the fluorimeter. For the luminescence lifetimes, an Edinburgh OB 900 timecorrelated single-photon-counting spectrometer was used. As excitation sources, a Hamamatsu PLP 2 laser diode (59 ps pulse width at $408 \mathrm{~nm}$ ) and/or the nitrogen discharge (pulse width $2 \mathrm{~ns}$ at $337 \mathrm{~nm}$ ) were employed. For luminescence quantum yield, the optically dilute method was employed, ${ }^{[\mathrm{S} 1]}$ using $\left[\mathrm{Ru}(\mathrm{bpy})_{3}\right]^{2+}$ in airequilibrated aqueous solution as a quantum yield standard $(\Phi=0.028)$. $\left.{ }^{\mathrm{S} 2}\right]$

Time-resolved transient absorption experiments were performed using a pumpprobe setup based on the Spectra-Physics MAI-TAI Ti:sapphire system as the laser source and the Ultrafast Systems Helios spectrometer as the detector. The output of laser beam was split to generate pump and probe beam pulses with a beam splitter (85 and 15\%). The pump pulse (400 nm, 1-2 $\mu \mathrm{J}$ ) was generated with a Spectra-Physics 800 FP OPA and was focused onto the sample cuvette. The probe beam was delayed with a computer controlled motion controller and then focused into a 2-mm sapphire plate to generate a white light continuum (spectral range 450-800 nm). The white light is then overlapped with the pump beam in a 2-mm quartz cuvette containing the sample. The effective time resolution was ca. $200 \mathrm{fs}$, and the temporal chirp over the white-light 450-750 nm range ca. $150 \mathrm{fs}$; the temporal window of the optical delay stage was 0-3200 ps. Please note that all the transient spectra shown in the present paper are chirp corrected. The chirp correction was done by using the pump-induced absorption signals themselves in the same conditions (same cuvette, solvent, temperature, stirring frequency, etc.) used for each single experiment. The timeresolved data were analyzed with the Ultrafast Systems Surface Explorer Pro software.

Experimental uncertainties are as follows: absorption maxima, $2 \mathrm{~nm}$; luminescence maxima, $4 \mathrm{~nm}$; luminescence lifetimes, 10\%; luminescence quantum yields, 15\%; transient absorption decay and rise rates, $10 \%$.

\section{Electrochemistry}

Electrochemical experiments were carried out with a conventional three-electrode cell and a PC-controlled potentiostat/galvanostat (Princeton Applied Research Inc. model 263A). The working electrode was a platinum electrode from Radiometer-Tacussel (area, $0.0314 \mathrm{~cm}^{2}$; diameter, $2.0 \mathrm{~mm}$ ) mounted in Teflon. Platinum wire was used as the counter-electrode and a saturated calomel electrode (SCE) as the reference. Electrolytic solutions, MeCN (Aldrich, anhydrous, $99.8 \%$ ) containing $0.1 \mathrm{M}$ tetrabutylammonium hexafluorophosphate $\left(\mathrm{TBAPF}_{6}\right.$, Aldrich, $+99 \%$ ) as a supporting electrolyte, were routinely deoxygenated by argon bubbling. All potential values are given versus the SCE. The reported numerical values were corrected 
by using a dissolved $\mathrm{Fc}^{+} / \mathrm{Fc}$ couple as an internal reference and by setting $\mathrm{E}_{1 / 2}\left(\mathrm{Fc}^{+} / \mathrm{Fc}\right)$ equal to $+0.380 \mathrm{~V}$ vs SCE in $\mathrm{MeCN}$. ${ }^{[33]}$ Cyclic voltammetry experiments were conducted at a scan rate of $0.1 \mathrm{~V} \mathrm{~s}^{-1}$. Experimental uncertainty on potential values is $10 \mathrm{mV}$. 


\section{Additional Figures for the photophysical properties}
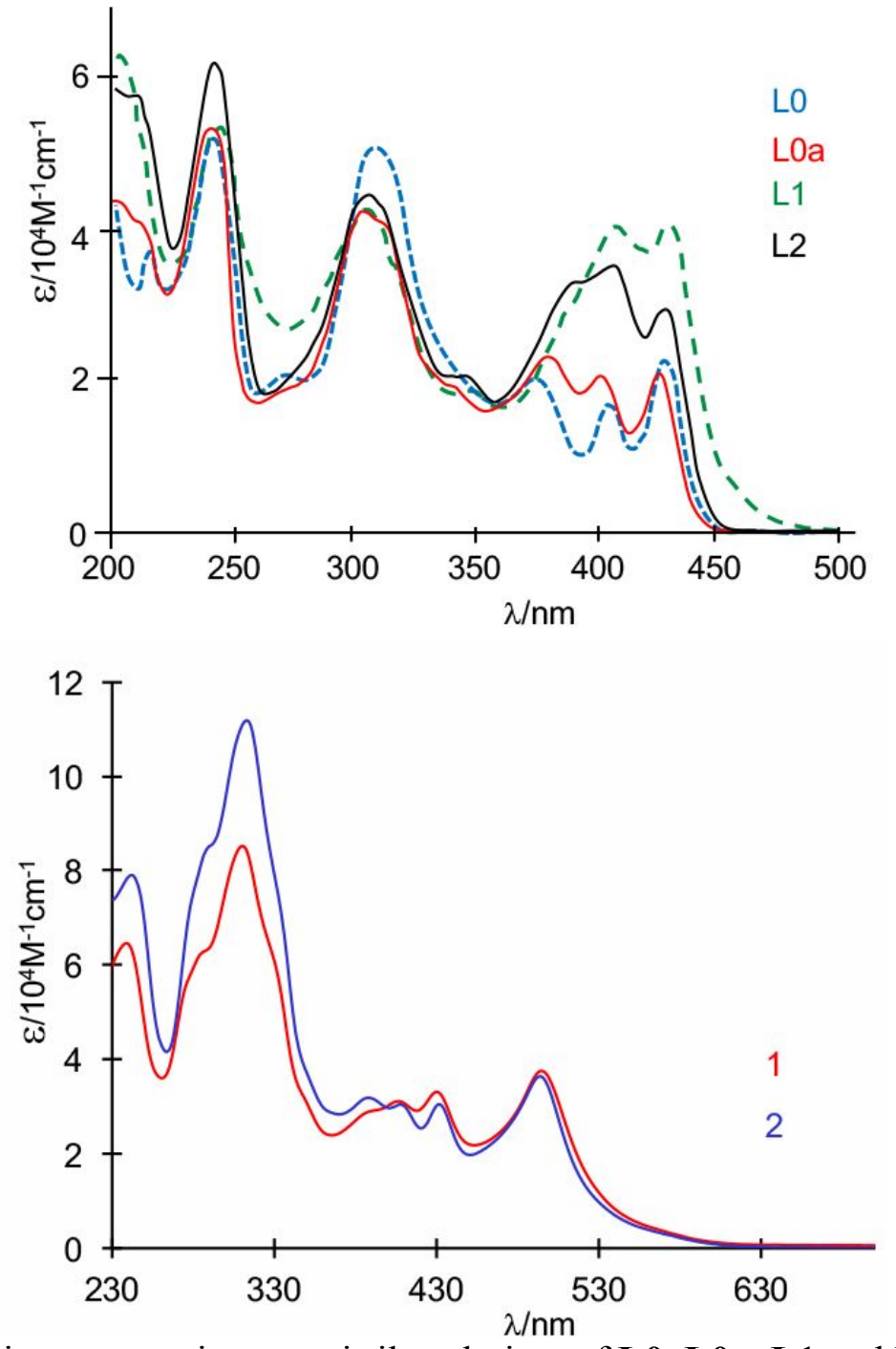

Figure S1. Absorption spectra, in acetonitrile solution, of L0, L0a, L1 and L2 (top panel). In the bottom panel the absorption spectra of $\mathbf{1}$ and $\mathbf{2}$, are reported. 

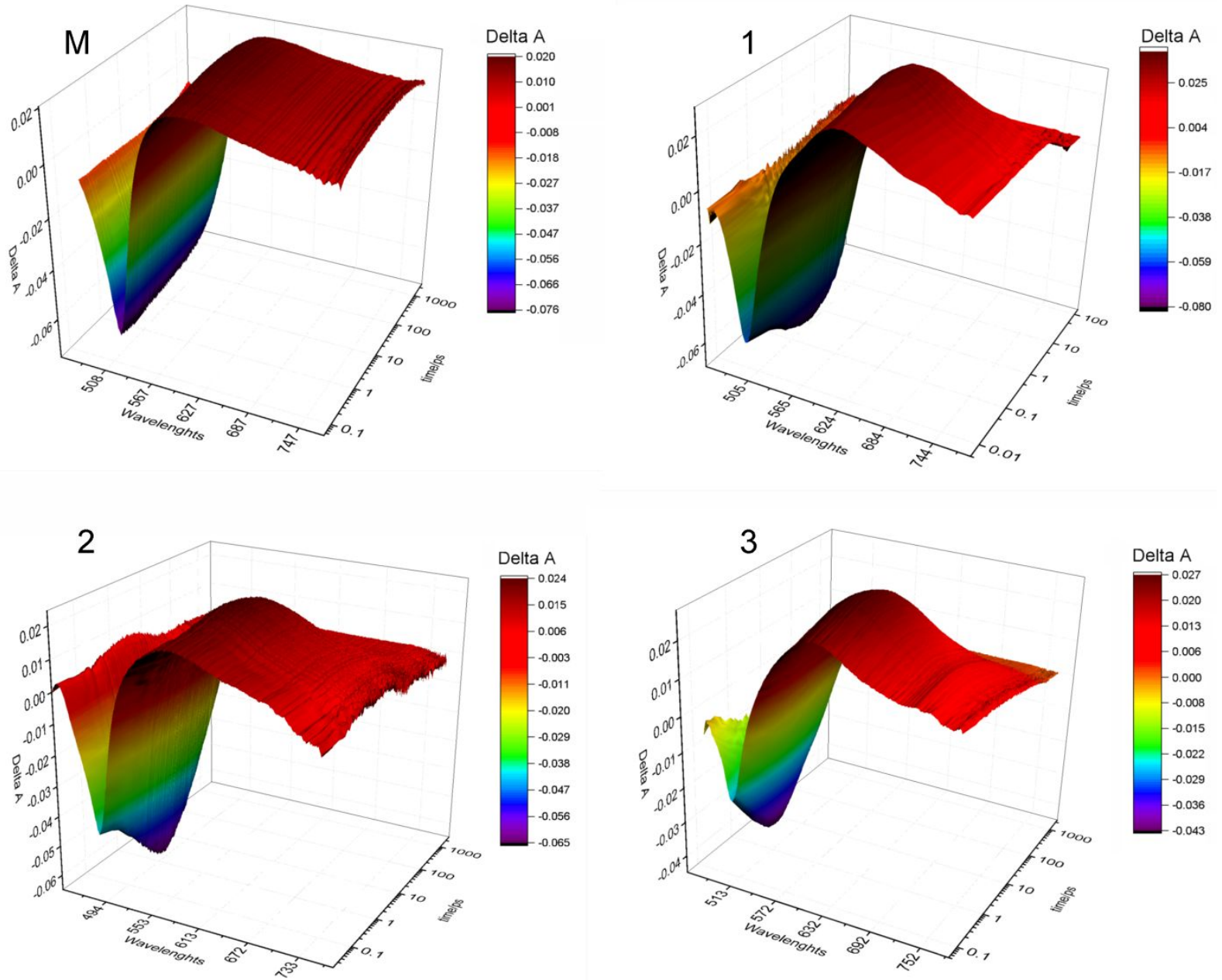

Figure S2. Transient absorption spectra matrices of M, 1, 2 and 3, in acetonitrile solution (excitation pulse @400 nm). Time scale is logarithmic. 


\section{Syntheses and Characterization.}

\section{General Experimental Details. Characterizations.}

${ }^{1} \mathrm{H}$ and ${ }^{13} \mathrm{C}$ NMR spectra were recorded on Bruker Avance II $500 \mathrm{MHz}$ and Bruker ARX 250 $\mathrm{MHz}$ spectrometers. Chemical shifts for ${ }^{1} \mathrm{H}$ NMR spectra are referenced relative to residual protium in the deuterated solvent (DMSO- $d^{6} \delta=2.50 \mathrm{ppm}$ and $\mathrm{CD}_{3} \mathrm{CN} \delta=1.94 \mathrm{ppm}$ ). Chemical shifts for ${ }^{13} \mathrm{C}$ NMR spectra are referenced relative to carbon in the deuterated solvent (DMSO- $d^{6} \delta=39.52 \mathrm{ppm}$ and $\mathrm{CD}_{3} \mathrm{CN} \delta=118.26$ and $1.32 \mathrm{ppm}$ ). When needed, NMR assignments were determined by bidimensional NMR experiments (COSY, HSQC and HMBC). Precursor compounds were also characterized by low-resolution electrospray ionization mass spectrometry (ESI-MS), using a LCQ Advantage ThermoFinnigan spectrometer (solvent, acetonitrile). High-resolution mass spectrometry was carried out to characterize target compounds only. These analyses were performed by flowinjection analysis by using a Surveyor pump and an autosampler. Each direct introduction analysis was carried out by injecting $20 \mu \mathrm{L}$ of sample at a flow rate of $100 \mu \mathrm{L} / \mathrm{min}$ of a mobile phase consisting of $\mathrm{MeOH} / \mathrm{MeCN}$ for the positive ion mode. The mass spectrometer was an LTQ-Orbitrap (Thermo Fisher Scientific, Les Ulis, France) fitted with a heated electrospray source. The molecular profiling ESI-MS was performed in the positive ion detection mode. Mass spectra were recorded from $110 \mathrm{Th}$ up to $1500 \mathrm{Th}$ using a mass resolution of $60.10^{3}$ FWHM at 400 Th in the Orbitrap analyzer. Each mass spectrum was the average of 20 scans. In the positive ion mode, the electrospray voltage was set to $5 \mathrm{kV}$, the capillary voltage to $40 \mathrm{~V}$, and the tube lens offset to $100 \mathrm{~V}$. The sheath and auxiliary gas flows (both nitrogen) were optimized at 40 and 15 (arbitrary units), and the drying gas temperature was set to $250{ }^{\circ} \mathrm{C}$. The maximum injection time was $500 \mathrm{~ms}$.

\section{Syntheses. Chemicals.}

Column chromatography was carried out either with neutral or activated basic alumina (Aldrich, brockmann I, standard grade, 150 mesh, $58 \AA$ ). High-purity commercial reagent grade products were used without further purification. When required, solutions were degassed under reduced pressure $(20 \mathrm{~mm} \mathrm{Hg}$ ) followed by argon bubbling; this cycle was repeated three times. Ethylene glycol dimethyl ether and ethylene glycol were freshly distilled over $\mathrm{CaH}_{2}$, then degassed with argon. Microwave irradiations were conducted in a focused microwave reactor CEM Discover at the power and for the time indicated. $\mathrm{Pd}(\mathrm{dppf}) \mathrm{Cl}_{2}$ is [1,1'-bis(diphenylphosphino)ferrocene]dichloropalladium(II), $\mathrm{Pd}_{2} \mathrm{dba}_{3}$ is tris(dibenzylideneacetone)dipalladium(0), MeOTf is methyl trifluoromethanesulfonate and S-PHOS is 2dicyclohexylphosphino-2',6'-dimethoxybiphenyl. $\quad 4$ '-[p-(neopentylglycolatoboron)phenyl]2,2':6',2"-terpyridine (neoB-ph-tpy), ${ }^{[\mathrm{S} 4]}$ 9-(4'-bromophenyl)-benzo[c]benzo[1,2]quinolizino[3,4,5,6-ija][1,6]naphthyridin-15-ium tetrafluoroborate $\left[\mathrm{dBQNTH}_{2}-\mathrm{ph}-\mathrm{Br}\right](\mathrm{BF} 4){ }^{[\mathrm{S} 5]}{ }^{9}$ (4'-bromo-3',5'-dimethylphenyl)-benzo[c]benzo[1,2]quinolizino[3,4,5,6ija]-[1,6]naphthyridin15-ium tetrafluoroborate $\left[\mathrm{dBQNTH}_{2}-\mathrm{xy}-\mathrm{Br}\right]\left(\mathrm{BF}_{4}\right),{ }^{[\mathrm{S} 6]} \quad\left[4^{\prime}-\left(p\right.\right.$-tolyl)-2, $2^{\prime}: 6^{\prime}, 2^{\prime \prime}-$ terpyridine]ruthenium trichloride, ${ }^{[\mathrm{S} 7]}$ were prepared according to the literature method.

Notice that reported yields for the syntheses of molecules of interest for electrochemistry, electronic absorption and emission studies, are not optimized (given values are underestimates) since our main concern was actually the very high purity required, especially for very sensitive photophysical analytical methods. 


\section{General reaction schemes.}
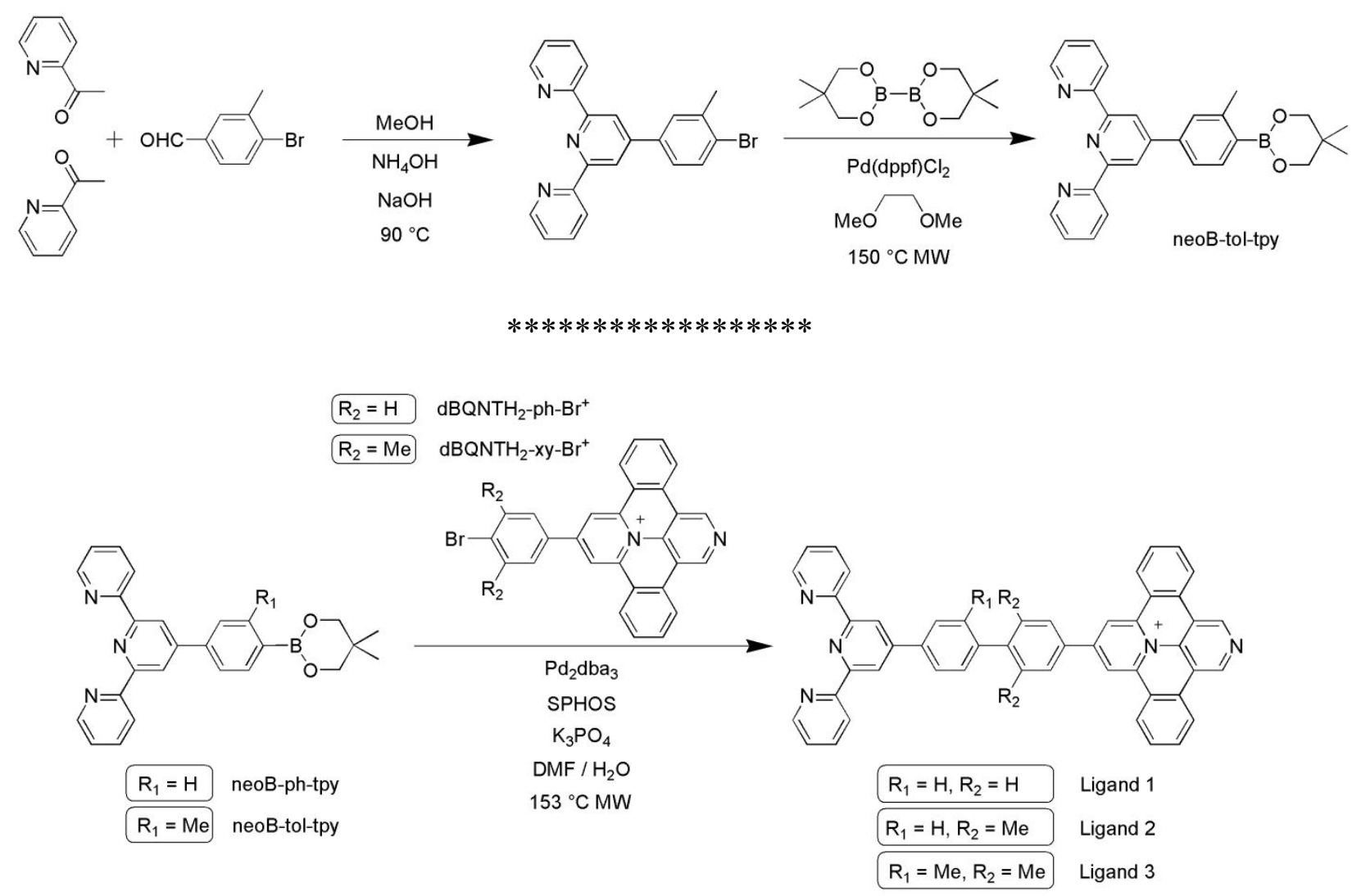

$* * * * * * * * * * * * * * * * * *$

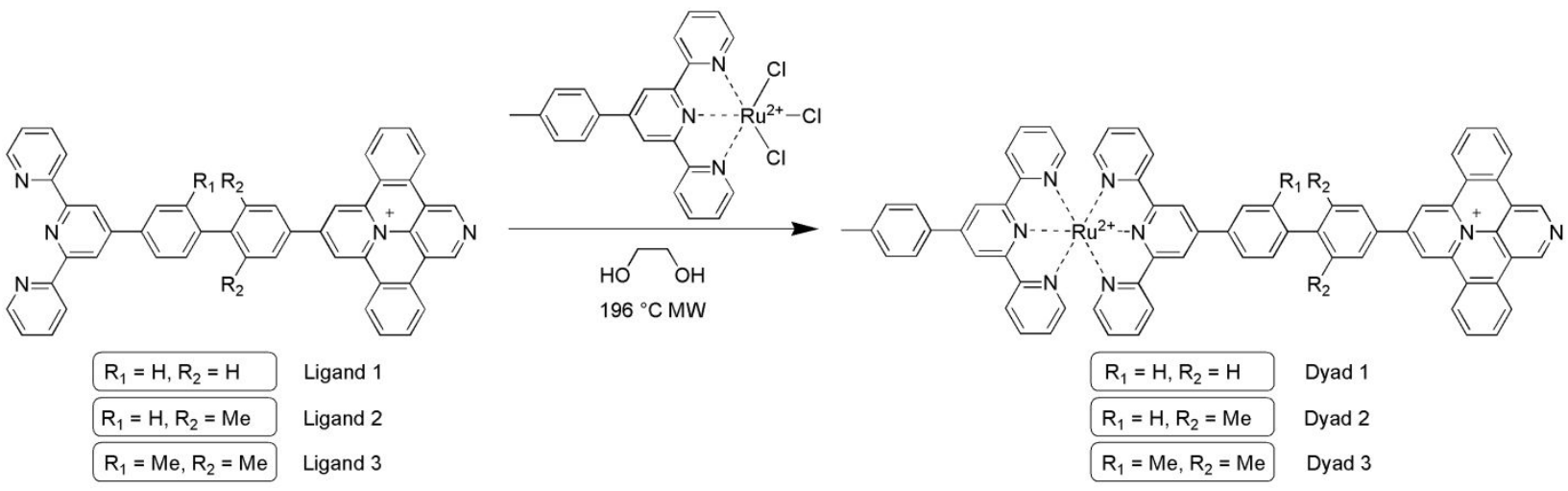

$* * * * * * * * * * * * * * * * * *$

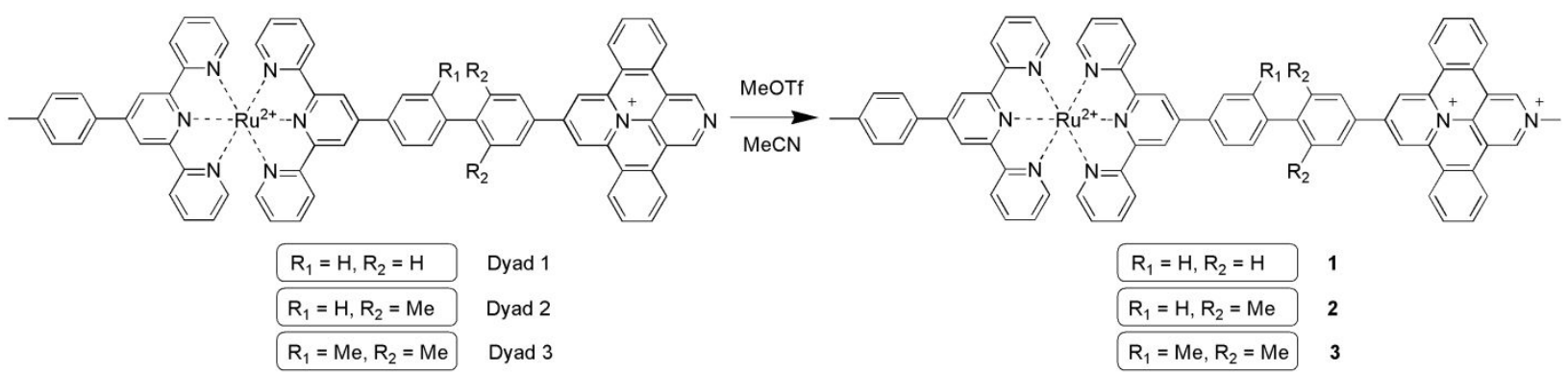




\section{Preparation of dyads $1,2,3$ and related precursors}

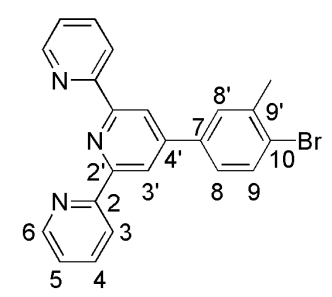

4'-(4-bromo-3-methylphenyl)-2,2':6',2"'-terpyridine

To a solution of 4-bromo-3-methylbenzaldehyde $(1.952 \mathrm{~g}, 9.81 \mathrm{mmol})$ and 2-acetylpyridine $(2.20 \mathrm{~mL}, 19.7 \mathrm{mmol})$ in $\mathrm{MeOH}(220 \mathrm{~mL})$ were added sodium hydroxide $(392 \mathrm{mg}, 9.81$ mmol) and an aqueous solution of ammonia $(55 \mathrm{~mL}, 26 \%)$. The solution was heated under reflux whilst stirring for 3 days under argon. Warning: $\mathrm{NH}_{3}$ gas evolution occurs for ca. $3 \mathrm{~h}$. The crude mixture was cooled to $0{ }^{\circ} \mathrm{C}$ and maintained at this temperature for additional 2 days, affording the expected product as a beige precipitate. This precipitate was filtered off, washed with a little amount of cold $\mathrm{MeOH}$ and dried in oven $(2.125 \mathrm{~g}, 54 \%)$. ${ }^{1} \mathrm{H}$ NMR (250 $\left.\mathrm{MHz}, \mathrm{CD}_{3} \mathrm{CN}\right): \delta 8.79-8.68\left(\mathrm{~m}, 6 \mathrm{H} ; \mathrm{H}^{6}, \mathrm{H}^{3}, \mathrm{H}^{3}\right) ; 7.96\left(\mathrm{td}, 2 \mathrm{H}, J=7.8,2.0 \mathrm{~Hz} ; \mathrm{H}^{4}\right) ; 7.82(\mathrm{~d}$, $\left.1 \mathrm{H}, J=2.0 \mathrm{~Hz} ; \mathrm{H}^{8}\right) ; 7.73\left(\mathrm{~d}, 1 \mathrm{H}, J=8.5 \mathrm{~Hz} ; \mathrm{H}^{9}\right) ; 7.60\left(\mathrm{dd}, 1 \mathrm{H}, J=8.5,2.5 \mathrm{~Hz} ; \mathrm{H}^{8}\right) ; 7.48$ 7.42(m, 2H; $\left.\mathrm{H}^{5}\right) ; 2.51\left(\mathrm{~s}, 3 \mathrm{H} ; \mathrm{H}^{\mathrm{Me}}\right)$. EI-MS: $m / z$ : calcd for $\mathrm{C}_{22} \mathrm{H}_{17} \mathrm{BrN}_{3}: 402.06\left[\mathrm{M}+\mathrm{H},{ }^{79} \mathrm{Br}\right]^{+}$, $404.06\left[\mathrm{M}+\mathrm{H},{ }^{81} \mathrm{Br}\right]^{+}$; found: $402.20\left[\mathrm{M}+\mathrm{H},{ }^{79} \mathrm{Br}\right]^{+}, 404.20\left[\mathrm{M}+\mathrm{H},{ }^{81} \mathrm{Br}\right]^{+}$.

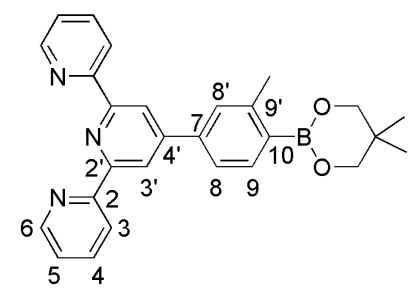

\section{4'-[3-methyl-4-(neopentylglycolatoboron)phenyl]-2,2':6',2"'-terpyridine}

A round bottom flask was charged with $\mathrm{Pd}(\mathrm{dppf}) \mathrm{Cl}_{2}(73 \mathrm{mg}, 0.1 \mathrm{mmol})$, anhydrous potassium acetate (439 mg, $4.48 \mathrm{mmol}$ ), bis(neopentylglycolato)diboron (354 mg, $1.57 \mathrm{mmol}$ ) and flushed with argon. Degassed anhydrous ethylene glycol dimethyl ether $(30 \mathrm{~mL})$ and 4'-(4bromo-3-methylphenyl)-2,2':6',2"-terpyridine $(600 \mathrm{mg}, 1.49 \mathrm{mmol})$ were then added. The mixture was heated at $150{ }^{\circ} \mathrm{C}$ in a microwave oven $(\mathrm{P}=500 \mathrm{~W})$ for $2 \mathrm{~h}$ whilst stirring under argon. $\mathrm{CH}_{2} \mathrm{Cl}_{2}(200 \mathrm{~mL})$ was added to the reaction crude and the organic layer was washed with water $(3 \times 200 \mathrm{~mL})$, dried over $\mathrm{MgSO}_{4}$ and concentrated under reduced pressure. The resulting residue was then washed with $\mathrm{Et}_{2} \mathrm{O}(10 \mathrm{~mL})$ in order to eliminate unreacted diboron. The supernatant was then removed with a pipette and the crude material finally evaporated under reduced pressure to afford the pure product as a beige solid (624 mg, 96\%). ${ }^{1} \mathrm{H}$ NMR $\left(250 \mathrm{MHz}, \mathrm{CD}_{3} \mathrm{CN}\right): \delta 8.76\left(\mathrm{~s}, 2 \mathrm{H} ; \mathrm{H}^{3}\right) ; 8.75-8.69\left(\mathrm{~m}, 4 \mathrm{H} ; \mathrm{H}^{3}, \mathrm{H}^{6}\right) ; 7.97(\mathrm{td}, 2 \mathrm{H}, J=7.8,1.5$ $\left.\mathrm{Hz} ; \mathrm{H}^{4}\right) ; 7.85\left(\mathrm{~d}, 1 \mathrm{H}, J=8.3 \mathrm{~Hz} ; \mathrm{H}^{9}\right) ; 7.70-7.66\left(\mathrm{~m}, 2 \mathrm{H} ; \mathrm{H}^{8}, \mathrm{H}^{8}\right) ; 7.47-7.43\left(\mathrm{~m}, 2 \mathrm{H} ; \mathrm{H}^{5}\right)$; $3.82\left(\mathrm{~s}, 4 \mathrm{H} ; \mathrm{H}^{\mathrm{CH} 2}\right) ; 2.62\left(\mathrm{~s}, 3 \mathrm{H} ; \mathrm{H}^{\mathrm{Me}}\right) ; 1.04\left(\mathrm{~s}, 6 \mathrm{H} ; \mathrm{H}^{\mathrm{Me}}\right)$, EI-MS: $m / z$ : calcd for $\mathrm{C}_{27} \mathrm{H}_{27} \mathrm{BN}_{3} \mathrm{O}_{2}$ : $435.22\left[\mathrm{M}+\mathrm{H},{ }^{10} \mathrm{~B}\right]^{+}, 436.22\left[\mathrm{M}+\mathrm{H},{ }^{11} \mathrm{~B}\right]^{+}$; found: $435.33\left[\mathrm{M}+\mathrm{H},{ }^{10} \mathrm{~B}\right]^{+}, 436.33\left[\mathrm{M}+\mathrm{H},{ }^{11} \mathrm{~B}\right]^{+}$. 


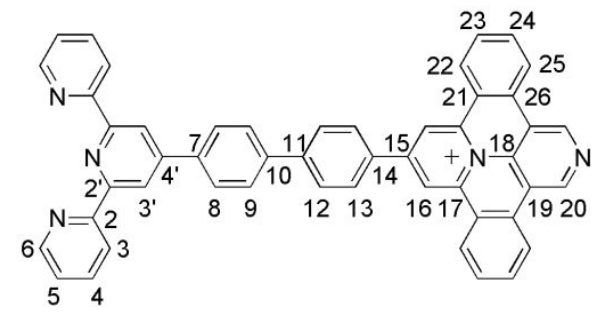

9-(4'-([2,2':6',2''-terpyridin]-4'-yl)-[1,1'-biphenyl]-4-yl)-benzo[c]benzo[1,2]quinolizino$[3,4,5,6-i j a][1,6]$ naphthyridin-15-ium hexafluorophosphate Ligand 1

A round bottom flask was charged with 4'-[p-(neopentylglycolatoboron)phenyl]-2,2':6',2"terpyridine (996 mg, $2.37 \mathrm{mmol}$ ), 9-(4'-bromophenyl)-benzo[c]benzo[1,2]quinolizino[3,4,5,6ija][1,6]naphthyridin-15-ium tetrafluoroborate $(863 \mathrm{mg}, 1.58 \mathrm{mmol})$, tripotassium phosphate (669 mg, $3.16 \mathrm{mmol}$ ), S-PHOS (168 mg, $0.41 \mathrm{mmol}), \mathrm{Pd}_{2} \mathrm{dba}_{3}(188 \mathrm{mg}, 0.21 \mathrm{mmol}$ ) and flushed with argon. Degassed DMF $(10 \mathrm{~mL})$ and $\mathrm{H}_{2} \mathrm{O}(1 \mathrm{~mL})$ were then added. The mixture was heated at $153{ }^{\circ} \mathrm{C}$ in a microwave oven $(\mathrm{P}=500 \mathrm{~W})$ for $20 \mathrm{~min}$ whilst stirring under argon. The solution became dark green, luminescent and viscous. The solvents were removed under reduced pressure. $\mathrm{CH}_{2} \mathrm{Cl}_{2}(200 \mathrm{~mL})$ and $\mathrm{H}_{2} \mathrm{O}(200 \mathrm{~mL})$ were added and the expected compound precipitated in the aqueous layer. Then the organic layer was removed with a separating funnel and the precipitate was filtered off. This solid was dissolved in DMSO (30 $\mathrm{mL}$ ) and that solution was slowly poured into a stirred aqueous solution of $\mathrm{KPF}_{6}(26 \mathrm{~g}$ in 650 $\mathrm{mL})$. The resulting precipitate was filtered off, dissolved again in DMSO (30 $\mathrm{mL})$ and that solution was slowly poured into a stirred solution of $\mathrm{Et}_{2} \mathrm{O}(500 \mathrm{~mL})$. Then, the solvents were removed with a pipette. The resulting solid was dissolved in acetone $(80 \mathrm{~mL})$ and reprecipitated by addition of $\mathrm{Et}_{2} \mathrm{O}(600 \mathrm{~mL})$. The precipitate was filtered off, washed with $\mathrm{EtOH}$ and $\mathrm{Et}_{2} \mathrm{O}$ and dried in oven to afford the pure product as a green solid $(1.14 \mathrm{~g}, 87 \%) .{ }^{1} \mathrm{H}$ NMR (500 MHz, DMSO- $\left.d^{6}\right): \delta 10.42\left(\mathrm{~s}, 2 \mathrm{H} ; \mathrm{H}^{20}\right) ; 9.87\left(\mathrm{~s}, 2 \mathrm{H} ; \mathrm{H}^{16}\right) ; 9.52(\mathrm{~d}, 2 \mathrm{H}, J=9.0 \mathrm{~Hz}$; $\left.\mathrm{H}^{22}\right) ; 9.17\left(\mathrm{~d}, 2 \mathrm{H}, J=8.5 \mathrm{~Hz} ; \mathrm{H}^{25}\right) ; 8.78-8.75\left(\mathrm{~m}, 4 \mathrm{H} ; \mathrm{H}^{13}, \mathrm{H}^{6}\right) ; 8.75\left(\mathrm{~s}, 2 \mathrm{H} ; \mathrm{H}^{3}\right) ; 8.64(\mathrm{~d}, 2 \mathrm{H}$, $\left.J=8.5 \mathrm{~Hz} ; \mathrm{H}^{3}\right) ; 8.21-8.17\left(\mathrm{~m}, 4 \mathrm{H} ; \mathrm{H}^{12}, \mathrm{H}^{24}\right) ; 8.12-8.09\left(\mathrm{~m}, 6 \mathrm{H} ; \mathrm{H}^{8}, \mathrm{H}^{9}, \mathrm{H}^{23}\right) ; 8.05(\mathrm{td}, 2 \mathrm{H}, J$ $\left.=7.5,1.0 \mathrm{~Hz} ; \mathrm{H}^{4}\right) ; 7.56-7.52\left(\mathrm{~m}, 2 \mathrm{H} ; \mathrm{H}^{5}\right) .{ }^{13} \mathrm{C}$ NMR $\left(126 \mathrm{MHz}, \mathrm{DMSO}-d^{6}\right): \delta 155.3\left(\mathrm{C}^{2}\right)$; $154.0\left(\mathrm{C}^{2}\right) ; 149.9\left(\mathrm{C}^{4}, \mathrm{C}^{15}\right) ; 148.7\left(\mathrm{C}^{6}\right) ; 144.8\left(\mathrm{C}^{20}\right) ; 142.9\left(\mathrm{C}^{17}\right) ; 142.2\left(\mathrm{C}^{11}\right) ; 139.1\left(\mathrm{C}^{10}\right)$; $137.0\left(\mathrm{C}^{4}\right) ; 136.4\left(\mathrm{C}^{7}\right) ; 133.9\left(\mathrm{C}^{24}\right) ; 132.5\left(\mathrm{C}^{14}\right) ; 131.3\left(\mathrm{C}^{18}\right) ; 130.8\left(\mathrm{C}^{23}\right) ; 129.6\left(\mathrm{C}^{13}\right) ; 127.5$ $\left(\mathrm{C}^{8}\right) ; 127.2\left(\mathrm{C}^{22}\right) ; 127.1\left(\mathrm{C}^{9 / 12}\right) ; 127.0\left(\mathrm{C}^{9 / 12}\right) ; 126.5\left(\mathrm{C}^{26}\right) ; 124.2\left(\mathrm{C}^{5}\right) ; 124.0\left(\mathrm{C}^{21}\right) ; 122.9$ $\left(\mathrm{C}^{25}\right) ; 120.0\left(\mathrm{C}^{3}\right) ; 117.5\left(\mathrm{C}^{16}, \mathrm{C}^{19}\right) ; 116.5\left(\mathrm{C}^{3}\right)$. EI-MS: $m / z$ : ca/lcd for $\mathrm{C}_{49} \mathrm{H}_{30} \mathrm{~N}_{5}: 688.25$ $\left[\mathrm{M}-\mathrm{PF}_{6}\right]^{+}$; found: $688.53\left[\mathrm{M}-\mathrm{PF}_{6}\right]^{+}$.

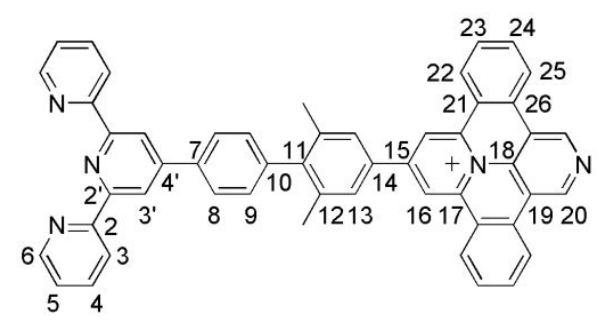

9-(4'-([2,2':6',2'-terpyridin]-4'-yl)-2,6-dimethyl-[1,1'-biphenyl]-4-yl)-benzo[c]benzo[1,2]quinolizino[3,4,5,6-ija][1,6]naphthyridin-15-ium hexafluorophosphate Ligand 2

A round bottom flask was charged with $4^{\prime}$-[p-(neopentylglycolatoboron)phenyl $]-2,2^{\prime}: 6^{\prime}, 2^{\prime \prime}-$ terpyridine $(858 \quad \mathrm{mg}, \quad 2.04 \quad \mathrm{mmol}), \quad 9-\left(4^{\prime}\right.$-bromo-3',5'-dimethylphenyl)benzo[c] benzo[1,2]quinolizino[3,4,5,6-ija][1,6]naphthyridin-15-ium tetrafluoroborate (758 $\mathrm{mg}, 1.32 \mathrm{mmol})$, tripotassium phosphate $(559 \mathrm{mg}, 2.64 \mathrm{mmol})$, S-PHOS (141 mg, 0.34 $\mathrm{mmol}), \mathrm{Pd}_{2} \mathrm{dba}_{3}(157 \mathrm{mg}, 0.17 \mathrm{mmol})$ and flushed with argon. Degassed DMF (8.4 mL) and $\mathrm{H}_{2} \mathrm{O}(0.84 \mathrm{~mL})$ were then added. The mixture was heated at $153^{\circ} \mathrm{C}$ in a microwave oven $(\mathrm{P}=$ $500 \mathrm{~W}$ ) for $20 \mathrm{~min}$ whilst stirring under argon. The solution became dark green, luminescent 
and viscous. The solvents were removed under reduced pressure. $\mathrm{CH}_{2} \mathrm{Cl}_{2}(200 \mathrm{~mL})$ and $\mathrm{H}_{2} \mathrm{O}$ $(200 \mathrm{~mL})$ were added and the expected compound precipitated in the aqueous layer. Then the organic layer was removed with a separating funnel and the precipitate was filtered off. This solid was dissolved in DMSO $(60 \mathrm{~mL})$ and that solution was slowly poured into a stirred aqueous solution of $\mathrm{KPF}_{6}(26 \mathrm{~g}$ in $650 \mathrm{~mL})$. The resulting precipitate was filtered off, washed with $\mathrm{Et}_{2} \mathrm{O}$ and $\mathrm{CH}_{3} \mathrm{CN}$. The solid was dissolved again in DMSO $(30 \mathrm{~mL})$ and that solution was slowly poured into a stirred solution of $\mathrm{Et}_{2} \mathrm{O}(500 \mathrm{~mL})$. The precipitate was filtered off, washed with $\mathrm{Et}_{2} \mathrm{O}$ and dried in oven to afford the pure product as a green solid $(600 \mathrm{mg}$, 53\%). ${ }^{1} \mathrm{H}$ NMR (500 MHz, DMSO- $\left.d^{6}\right): \delta 10.44\left(\mathrm{~s}, 2 \mathrm{H} ; \mathrm{H}^{20}\right) ; 9.85\left(\mathrm{~s}, 2 \mathrm{H} ; \mathrm{H}^{16}\right) ; 9.51(\mathrm{~d}, 2 \mathrm{H}, J$ $\left.=8.5 \mathrm{~Hz} ; \mathrm{H}^{22}\right) ; 9.21\left(\mathrm{~d}, 2 \mathrm{H}, J=8.0 \mathrm{~Hz} ; \mathrm{H}^{25}\right) ; 8.83\left(\mathrm{~s}, 2 \mathrm{H} ; \mathrm{H}^{3}\right) ; 8.78\left(\mathrm{~d}, 2 \mathrm{H}, J=4.0 \mathrm{~Hz} ; \mathrm{H}^{3}\right)$; $8.71\left(\mathrm{~d}, 2 \mathrm{H}, J=7.5 \mathrm{~Hz} ; \mathrm{H}^{6}\right) ; 8.46\left(\mathrm{~s}, 2 \mathrm{H} ; \mathrm{H}^{13}\right) ; 8.23\left(\mathrm{t}, 2 \mathrm{H}, J=7.5 \mathrm{~Hz} ; \mathrm{H}^{24}\right) ; 8.24-8.10(\mathrm{~m}$, $\left.4 \mathrm{H} ; \mathrm{H}^{8}, \mathrm{H}^{23}\right) ; 8.07\left(\mathrm{td}, 2 \mathrm{H}, J=7.5,1.5 \mathrm{~Hz} ; \mathrm{H}^{4}\right) ; 7.58-7.54\left(\mathrm{~m}, 2 \mathrm{H} ; \mathrm{H}^{5}\right) ; 7.51(\mathrm{~d}, 2 \mathrm{H}, J=8.5$ $\left.\mathrm{Hz} ; \mathrm{H}^{9}\right) ; 2.32$ (s, $\left.6 \mathrm{H} ; \mathrm{H}^{\mathrm{Me}(12)}\right) .{ }^{13} \mathrm{C}$ NMR $\left(126 \mathrm{MHz}, \mathrm{DMSO}-d^{6}\right): \delta 155.5 ; 154.5 ; 150.5 ; 149.2$; $148.9 ; 145.0 ; 144.9 ; 143.2 ; 140.5 ; 137.4 ; 137.2 ; 136.3 ; 134.3 ; 132.4 ; 131.7 ; 131.1 ; 129.6$; $128.0 ; 127.5 ; 127.3 ; 126.8 ; 124.5 ; 124.4 ; 123.2 ; 120.7 ; 117.8 ; 117.7 ; 20.7\left(\mathrm{C}^{\mathrm{Me}(12)}\right)$. EI-MS: $m / z$ : calcd for $\mathrm{C}_{51} \mathrm{H}_{34} \mathrm{~N}_{5}: 716.28\left[\mathrm{M}-\mathrm{PF}_{6}\right]^{+}$; found: $716.47\left[\mathrm{M}-\mathrm{PF}_{6}\right]^{+}$.

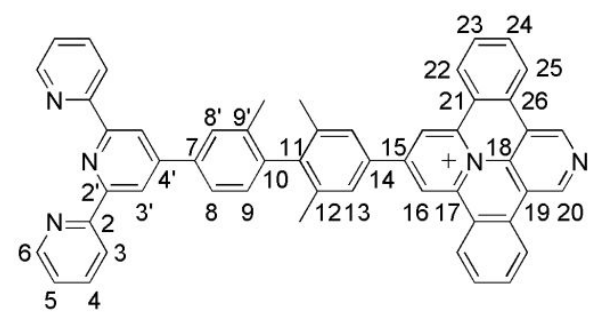

9-(4'-([2,2':6',2"'-terpyridin]-4'-yl)-2,2',6-trimethyl-[1,1'-biphenyl]-4-yl)-benzo[c]benzo[1,2]quinolizino[3,4,5,6-ija][1,6]naphthyridin-15-ium tetrafluoroborate Ligand 3

A round bottom flask was charged with 4'-[3-methyl-4-(neopentylglycolatoboron)phenyl]2,2':6',2"-terpyridine (518 mg, $1.19 \mathrm{mmol}), \quad 9$-(4'-bromo-3',5'-dimethylphenyl)benzo[c]benzo[1,2]quinolizino[3,4,5,6-ija][1,6]naphthyridin-15-ium tetrafluoroborate (456 $\mathrm{mg}, 0.79 \mathrm{mmol})$, tripotassium phosphate (336 mg, $1.59 \mathrm{mmol})$, S-PHOS ( $85 \mathrm{mg}, 0.21 \mathrm{mmol}$ ), $\mathrm{Pd}_{2} \mathrm{dba}_{3}(94 \mathrm{mg}, 0.10 \mathrm{mmol})$ and flushed with argon. Degassed DMF (5.0 mL) and $\mathrm{H}_{2} \mathrm{O}(0.5$ $\mathrm{mL})$ were then added. The mixture was heated at $153{ }^{\circ} \mathrm{C}$ in a microwave oven $(\mathrm{P}=500 \mathrm{~W})$ for 25 min whilst stirring under argon. The solution became dark green, luminescent and viscous. The solvents were removed under reduced pressure and $\mathrm{CH}_{2} \mathrm{Cl}_{2}(200 \mathrm{~mL})$ and $\mathrm{H}_{2} \mathrm{O}$ $(200 \mathrm{~mL})$ were added. A part of the expected compound precipitated in the aqueous layer and the other part remained in the organic layer. Then the organic layer was isolated with a separating funnel, washed with $\mathrm{H}_{2} \mathrm{O}$, dried over $\mathrm{MgSO}_{4}$ and concentrated under reduced pressure, affording a dark green solid. This solid was purified by column chromatography over neutral alumina $\left(\mathrm{CH}_{2} \mathrm{Cl}_{2} / \mathrm{CH}_{3} \mathrm{OH}, 99 / 1\right.$ to $\left.98 / 2\right)$ to yield a green solid which is not pure. Then this solid was purified a second time by column chromatography over neutral alumina $\left(\mathrm{CH}_{2} \mathrm{Cl}_{2}\right.$ /acetone, $100 / 0$ to $\left.0 / 100\right)$ to yield a yellow solid, which was reprecipitated in $\mathrm{Et}_{2} \mathrm{O}$ $(217 \mathrm{mg})$. The aqueous layer was filtered to isolate the precipitate. This solid was dissolved in DMSO $(30 \mathrm{~mL})$ and that solution was slowly poured into a stirred aqueous solution of $\mathrm{NH}_{4} \mathrm{BF}_{4}\left(26 \mathrm{~g}\right.$ in $650 \mathrm{~mL}$ ). The precipitate was filtered off, washed with $\mathrm{H}_{2} \mathrm{O}$ and $\mathrm{Et}_{2} \mathrm{O}$, yielding a yellow solid $(263 \mathrm{mg})$. Finally, $480 \mathrm{mg}$ of the pure product were obtained as a yellow solid (74\%). ${ }^{1} \mathrm{H}$ NMR (500 MHz, DMSO-d $\left.d^{6}\right): \delta 10.45\left(\mathrm{~s}, 2 \mathrm{H} ; \mathrm{H}^{20}\right) ; 9.88\left(\mathrm{~s}, 2 \mathrm{H} ; \mathrm{H}^{16}\right)$; $9.53\left(\mathrm{~d}, 2 \mathrm{H}, J=8.5 \mathrm{~Hz} ; \mathrm{H}^{22}\right) ; 9.22\left(\mathrm{~d}, 2 \mathrm{H}, J=8.0 \mathrm{~Hz} ; \mathrm{H}^{25}\right) ; 8.83\left(\mathrm{~s}, 2 \mathrm{H} ; \mathrm{H}^{3}\right) ; 8.79$ (d, $2 \mathrm{H}, J=$ $\left.4.5 \mathrm{~Hz} ; \mathrm{H}^{3}\right) ; 8.72\left(\mathrm{~d}, 2 \mathrm{H}, J=7.5 \mathrm{~Hz} ; \mathrm{H}^{6}\right) ; 8.47\left(\mathrm{~s}, 2 \mathrm{H} ; \mathrm{H}^{13}\right) ; 8.23\left(\mathrm{t}, 2 \mathrm{H}, J=7.5 \mathrm{~Hz} ; \mathrm{H}^{24}\right) ; 8.12$ (t, $\left.2 \mathrm{H}, J=7.5 \mathrm{~Hz} ; \mathrm{H}^{23}\right) ; 8.08\left(\mathrm{td}, 2 \mathrm{H}, J=8.5,1.0 \mathrm{~Hz} ; \mathrm{H}^{4}\right) ; 8.03\left(\mathrm{~s}, 1 \mathrm{H} ; \mathrm{H}^{8}\right) ; 7.95(\mathrm{~d}, 1 \mathrm{H}, J=$ $\left.7.5 \mathrm{~Hz} ; \mathrm{H}^{8}\right) ; 7.58-7.55\left(\mathrm{~m}, 2 \mathrm{H} ; \mathrm{H}^{5}\right) ; 7.33\left(\mathrm{~d}, 1 \mathrm{H}, J=8.0 \mathrm{~Hz} ; \mathrm{H}^{9}\right) ; 2.24\left(\mathrm{~s}, 6 \mathrm{H} ; \mathrm{H}^{\mathrm{Me}(12)}\right) ; 2.20$ (s, $\left.3 \mathrm{H} ; \mathrm{H}^{\mathrm{Me}\left(9^{\prime}\right)}\right)$. EI-MS: $m / z$ : calcd for $\mathrm{C}_{52} \mathrm{H}_{36} \mathrm{~N}_{5}$ : $730.30\left[\mathrm{M}-\mathrm{BF}_{4}\right]^{+}$; found: $730.60\left[\mathrm{M}-\mathrm{BF}_{4}\right]^{+}$. 


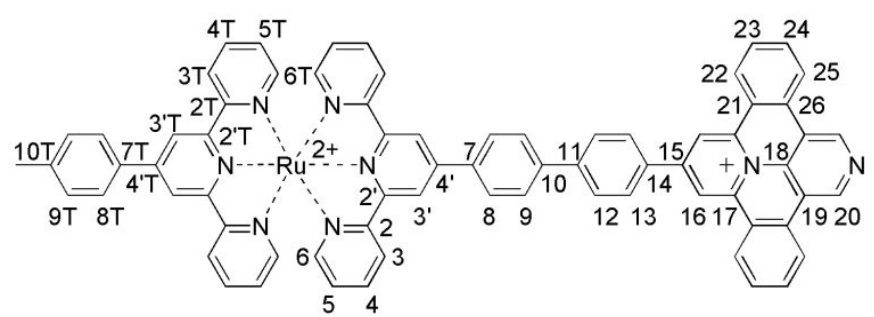

Dyad 1: [(ttpy)Ru(tpy-Ph 2 -dBQNTH 2$)]\left(\mathrm{PF}_{6}\right)_{3}$

A solution of [ $4^{\prime}$-(p-tolyl)-2,2': $6^{\prime}, 2^{\prime \prime}$-terpyridine]ruthenium trichloride $(212 \mathrm{mg}, 0.40 \mathrm{mmol})$ and 9-(4'-([2,2':6',2"-terpyridin]-4'-yl)-[1,1'-biphenyl]-4-yl)-benzo[c]benzo[1,2]quinolizino[3,4,5,6-ija][1,6]naphthyridin-15-ium hexafluorophosphate ligand 1 (400 $\mathrm{mg}, 0.48 \mathrm{mmol}$ ) in anhydrous degassed ethylene glycol $(39 \mathrm{~mL})$ was sonicated for $2 \mathrm{~min}$ and then heated at 196 ${ }^{\circ} \mathrm{C}$ in a microwave oven $(\mathrm{P}=500 \mathrm{~W})$ for $10 \mathrm{~min}$ whilst stirring. The solution became brown. Absolute EtOH $(28 \mathrm{~mL}), \mathrm{NH}_{4} \mathrm{PF}_{6}(1.4 \mathrm{~g})$ and $\mathrm{H}_{2} \mathrm{O}(70 \mathrm{~mL})$ were added to the reaction mixture which was then stirred for 10 min. This solid was filtered off and washed with $\mathrm{H}_{2} \mathrm{O}$ to remove solvents and excess salts. The solid was dissolved in acetone $(150 \mathrm{~mL})$ and $\mathrm{EtOH}(50$ $\mathrm{mL}$ ) was added. Acetone was removed under reduced pressure and the product precipitated in EtOH. The precipitate was filtered off and washed with $\mathrm{Et}_{2} \mathrm{O}$. The resulting solid was purified by column chromatography over basic alumina $\left(\mathrm{CH}_{2} \mathrm{Cl}_{2}\right.$ /acetone, $100 / 0$ to $0 / 100$ and acetone $/ \mathrm{CH}_{3} \mathrm{CN}, 100 / 0$ to $\left.90 / 10\right)$ to yield a red solid (149 mg), which was reprecipitated in a mixture of acetone $/ \mathrm{Et}_{2} \mathrm{O}$. The resulting mixture was purified again by column chromatography over basic alumina $\left(\mathrm{CH}_{2} \mathrm{Cl}_{2}\right.$ /acetone, $100 / 0$ to $\left.0 / 100\right)$ to yield a red solid, which was recrystallized ten times by slow diffusion of $\mathrm{Et}_{2} \mathrm{O}$ in a concentrated $\mathrm{CH}_{3} \mathrm{CN}$ solution of the complex (76 mg, 12\%). ${ }^{1} \mathrm{H}$ NMR $\left(500 \mathrm{MHz}, \mathrm{CD}_{3} \mathrm{CN}\right): \delta 10.27\left(\mathrm{~s}, 2 \mathrm{H} ; \mathrm{H}^{20}\right)$; $9.67\left(\mathrm{~s}, 2 \mathrm{H} ; \mathrm{H}^{16}\right) ; 9.21\left(\mathrm{~d}, 2 \mathrm{H}, J=8.0 \mathrm{~Hz} ; \mathrm{H}^{22}\right) ; 9.10\left(\mathrm{~s}, 2 \mathrm{H} ; \mathrm{H}^{3}\right) ; 9.04-9.01\left(\mathrm{~m}, 4 \mathrm{H} ; \mathrm{H}^{25}\right.$, $\left.\mathrm{H}^{3^{\prime} \mathrm{T}}\right) ; 8.70\left(\mathrm{~d}, 2 \mathrm{H}, J=8.0 \mathrm{~Hz} ; \mathrm{H}^{3}\right) ; 8.66\left(\mathrm{~d}, 2 \mathrm{H}, J=8.0 \mathrm{~Hz} ; \mathrm{H}^{3 \mathrm{~T}}\right) ; 8.58(\mathrm{~d}, 2 \mathrm{H}, J=8.0 \mathrm{~Hz}$; $\left.\mathrm{H}^{13}\right) ; 8.42\left(\mathrm{~d}, 2 \mathrm{H}, J=8.0 \mathrm{~Hz} ; \mathrm{H}^{8}\right) ; 8.28-8.22\left(\mathrm{~m}, 6 \mathrm{H} ; \mathrm{H}^{9}, \mathrm{H}^{12}, \mathrm{H}^{24}\right) ; 8.14-8.11\left(\mathrm{~m}, 4 \mathrm{H} ; \mathrm{H}^{8 \mathrm{~T}}\right.$, $\left.\mathrm{H}^{23}\right) ; 7.99-7.95\left(\mathrm{~m}, 4 \mathrm{H} ; \mathrm{H}^{4}, \mathrm{H}^{4 \mathrm{~T}}\right) ; 7.60\left(\mathrm{~d}, 2 \mathrm{H}, J=7.5 \mathrm{~Hz} ; \mathrm{H}^{9 \mathrm{~T}}\right) ; 7.47-7.45\left(\mathrm{~m}, 4 \mathrm{H} ; \mathrm{H}^{6}, \mathrm{H}^{6 \mathrm{~T}}\right)$; 7.23-7.18 (m, 4H; $\left.\mathrm{H}^{5}, \mathrm{H}^{5 \mathrm{~T}}\right) ; 2.55\left(\mathrm{~s}, 3 \mathrm{H} ; \mathrm{H}^{\mathrm{Me}(10 \mathrm{~T})}\right) .{ }^{13} \mathrm{C} \mathrm{NMR}\left(126 \mathrm{MHz}, \mathrm{CD}_{3} \mathrm{CN}\right): \delta 159.3$ $\left(\mathrm{C}^{2 \mathrm{~T}}\right) ; 159.2\left(\mathrm{C}^{2}\right) ; 156.7\left(\mathrm{C}^{2}\right) ; 156.4\left(\mathrm{C}^{2} \mathrm{~T}\right) ; 153.5\left(\mathrm{C}^{6}, \mathrm{C}^{6 \mathrm{~T}}\right) ; 152.2\left(\mathrm{C}^{15}\right) ; 149.5\left(\mathrm{C}^{4} \mathrm{~T}\right) ; 148.3$ $\left(\mathrm{C}^{4}\right) ; 146.3\left(\mathrm{C}^{20}\right) ; 144.9\left(\mathrm{C}^{17}\right) ; 144.4\left(\mathrm{C}^{11}\right) ; 142.2\left(\mathrm{C}^{10 \mathrm{~T}}\right) ; 142.1\left(\mathrm{C}^{10}\right) ; 139.1\left(\mathrm{C}^{4,4 \mathrm{~T}}\right) ; 137.9$ $\left(\mathrm{C}^{7}\right) ; 135.8\left(\mathrm{C}^{24}\right)$; $135.1\left(\mathrm{C}^{14}\right) ; 134.8\left(\mathrm{C}^{7 \mathrm{~T}}\right) ; 133.4\left(\mathrm{C}^{18}\right)$; $132.4\left(\mathrm{C}^{23}\right) ; 131.4\left(\mathrm{C}^{9 \mathrm{~T}}\right) ; 130.8\left(\mathrm{C}^{13}\right)$; $129.5\left(\mathrm{C}^{8}\right) ; 129.4\left(\mathrm{C}^{9,12}\right) ; 128.7\left(\mathrm{C}^{8 \mathrm{~T}}\right) ; 128.5\left(\mathrm{C}^{5,5 \mathrm{~T}}\right) ; 128.4\left(\mathrm{C}^{26}\right) ; 128.3\left(\mathrm{C}^{22}\right) ; 125.7\left(\mathrm{C}^{21}\right)$; $125.6\left(\mathrm{C}^{3,3 \mathrm{~T}}\right) ; 124.3\left(\mathrm{C}^{25}\right) ; 122.5\left(\mathrm{C}^{3}\right) ; 122.4\left(\mathrm{C}^{3^{\prime} \mathrm{T}}\right) ; 120.1\left(\mathrm{C}^{16}\right) ; 119.3\left(\mathrm{C}^{19}\right) ; 21.3\left(\mathrm{C}^{\mathrm{Me}}\right)$, FTMS: $m / z$ : calcd for $\mathrm{C}_{71} \mathrm{H}_{47} \mathrm{~N}_{8} \mathrm{Ru}: 371.0990\left[\mathrm{M}-3 \mathrm{PF}_{6}\right]^{3+}$; found: $371.0995\left[\mathrm{M}-3 \mathrm{PF}_{6}\right]^{3+}$.

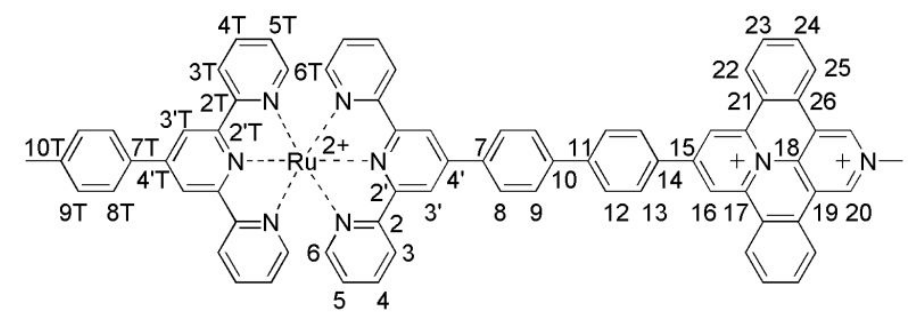

1: $\left[(\right.$ ttpy $\left.) \mathrm{Ru}\left(\mathrm{tpy}-\mathrm{Ph}_{2}-\mathrm{MedBQNTH} \mathbf{H}_{2}\right)\right]\left(\mathrm{PF}_{6}\right)_{4}$

To a solution of dyad $1(29 \mathrm{mg}, 0.020 \mathrm{mmol})$ in $\mathrm{MeCN}(60 \mathrm{~mL})$ was added MeOTf $(0.5 \mathrm{~mL}$, $4.56 \mathrm{mmol}$ ) under argon. The mixture was stirred for $30 \mathrm{~min}$ under argon. The reaction crude was then slowly transferred into a stirred aqueous solution of $\mathrm{KPF}_{6}(6.0 \mathrm{~g}$ in $150 \mathrm{~mL})$. The precipitate of the $\mathrm{PF}_{6}{ }^{-}$complex was filtered off and washed with $\mathrm{H}_{2} \mathrm{O}$ and $\mathrm{Et}_{2} \mathrm{O}$. The solid is a mixture of the starting material $(77 \%$ molar) and the expected product $(23 \%$ molar). This procedure was repeated 7 times with a more concentrated solution $(25 \mathrm{~mL}$ of $\mathrm{MeCN})$. For 
each procedure, the product was precipitated in an aqueous solution of $\mathrm{KPF}_{6}(6.0 \mathrm{~g}$ in 150 $\mathrm{mL}$ ). The resulting brown precipitate was filtered off and washed with $\mathrm{H}_{2} \mathrm{O}$ and $\mathrm{Et}_{2} \mathrm{O}$, yielding the pure product as a red solid $(15 \mathrm{mg}, 43.9 \%) .{ }^{1} \mathrm{H} \mathrm{NMR}\left(500 \mathrm{MHz}, \mathrm{CD}_{3} \mathrm{CN}\right): \delta$ $10.17\left(\mathrm{~s}, 2 \mathrm{H} ; \mathrm{H}^{20}\right) ; 9.83\left(\mathrm{~s}, 2 \mathrm{H} ; \mathrm{H}^{16}\right) ; 9.35\left(\mathrm{~d}, 2 \mathrm{H}, J=8.5 \mathrm{~Hz} ; \mathrm{H}^{22}\right) ; 9.10\left(\mathrm{~s}, 2 \mathrm{H} ; \mathrm{H}^{3}\right) ; 9.02$ (s, $\left.2 \mathrm{H} ; \mathrm{H}^{3} \mathrm{\top}\right) ; 8.91\left(\mathrm{~d}, 2 \mathrm{H}, J=8.0 \mathrm{~Hz} ; \mathrm{H}^{25}\right) ; 8.70\left(\mathrm{~d}, 2 \mathrm{H}, J=9.0 \mathrm{~Hz} ; \mathrm{H}^{3}\right) ; 8.68-8.65(\mathrm{~m}, 4 \mathrm{H}$; $\left.\mathrm{H}^{3 \mathrm{~T}, 13}\right) ; 8.43\left(\mathrm{~d}, 2 \mathrm{H}, J=9.0 \mathrm{~Hz} ; \mathrm{H}^{8}\right) ; 8.38\left(\mathrm{t}, 2 \mathrm{H}, J=7.0 \mathrm{~Hz} ; \mathrm{H}^{24}\right) ; 8.34-8.30\left(\mathrm{~m}, 4 \mathrm{H} ; \mathrm{H}^{12}\right.$, $\left.\mathrm{H}^{23}\right) ; 8.27\left(\mathrm{~d}, 2 \mathrm{H}, J=8.0 \mathrm{~Hz} ; \mathrm{H}^{9}\right) ; 8.12\left(\mathrm{~d}, 2 \mathrm{H}, J=8.0 \mathrm{~Hz} ; \mathrm{H}^{8 \mathrm{~T}}\right) ; 8.00-7.95\left(\mathrm{~m}, 4 \mathrm{H} ; \mathrm{H}^{4}, \mathrm{H}^{4 \mathrm{~T}}\right)$; $7.60\left(\mathrm{~d}, 2 \mathrm{H}, J=7.5 \mathrm{~Hz} ; \mathrm{H}^{9 \mathrm{~T}}\right) ; 7.48-7.44\left(\mathrm{~m}, 4 \mathrm{H} ; \mathrm{H}^{6,6 \mathrm{~T}}\right) ; 7.23-7.18\left(\mathrm{~m}, 4 \mathrm{H} ; \mathrm{H}^{5}, \mathrm{H}^{5 \mathrm{~T}}\right) ; 4.86(\mathrm{~s}$, $\left.3 \mathrm{H} ; \mathrm{H}^{\mathrm{Me}(\mathrm{N})}\right) ; 2.55\left(\mathrm{~s}, 3 \mathrm{H} ; \mathrm{H}^{\mathrm{Me}(10 \mathrm{~T})}\right) .{ }^{13} \mathrm{C} \mathrm{NMR}\left(126 \mathrm{MHz}, \mathrm{CD}_{3} \mathrm{CN}\right): \delta 159.6\left(\mathrm{C}^{2,2 \mathrm{~T}}\right) ; 157.2$ $\left(\mathrm{C}^{2}\right) ; 156.7\left(\mathrm{C}^{2} \mathrm{~T}\right) ; 155.0\left(\mathrm{C}^{15}\right) ; 153.7\left(\mathrm{C}^{6,6 \mathrm{~T}}\right) ; 149.9\left(\mathrm{C}^{4} \mathrm{~T}\right) ; 148.9\left(\mathrm{C}^{4}\right) ; 145.6\left(\mathrm{C}^{11}\right) ; 145.0$ $\left(\mathrm{C}^{17}\right) ; 142.3\left(\mathrm{C}^{10 \mathrm{~T}}\right) ; 141.8\left(\mathrm{C}^{10}\right) ; 141.5\left(\mathrm{C}^{20}\right) ; 139.2\left(\mathrm{C}^{4,4 \mathrm{~T}}\right) ; 138.3\left(\mathrm{C}^{7}\right) ; 136.7\left(\mathrm{C}^{24}\right) ; 135.8$ $\left(\mathrm{C}^{18}\right) ; 135.1\left(\mathrm{C}^{7 \mathrm{~T}}\right) ; 135.0\left(\mathrm{C}^{23}\right) ; 134.7\left(\mathrm{C}^{14}\right) ; 131.5\left(\mathrm{C}^{13}\right) ; 131.4\left(\mathrm{C}^{9 \mathrm{~T}}\right) ; 129.7\left(\mathrm{C}^{8}\right) ; 129.6\left(\mathrm{C}^{9,12}\right)$; $129.2\left(\mathrm{C}^{22}\right) ; 128.9\left(\mathrm{C}^{8 \mathrm{~T}}\right) ; 128.4\left(\mathrm{C}^{5,5 \mathrm{~T}}\right) ; 126.9\left(\mathrm{C}^{21}\right) ; 126.0\left(\mathrm{C}^{26}\right) ; 125.7\left(\mathrm{C}^{3,3 \mathrm{~T}}\right) ; 125.2\left(\mathrm{C}^{25}\right)$; $124.8\left(\mathrm{C}^{19}\right) ; 122.6\left(\mathrm{C}^{3^{\prime}, 3^{\prime} \mathrm{T}}\right) ; 121.3\left(\mathrm{C}^{16}\right) ; 50.8\left(\mathrm{C}^{\mathrm{Me}(\mathrm{N})}\right) ; 21.4\left(\mathrm{C}^{\mathrm{Me}(10 \mathrm{~T})}\right)$, FTMS: $m / z$ : calcd for $\mathrm{C}_{72} \mathrm{H}_{50} \mathrm{~N}_{8} \mathrm{Ru}: 282.0800\left[\mathrm{M}-4 \mathrm{PF}_{6}\right]^{4+}$; found: $282.0791\left[\mathrm{M}-4 \mathrm{PF}_{6}\right]^{4+}$.

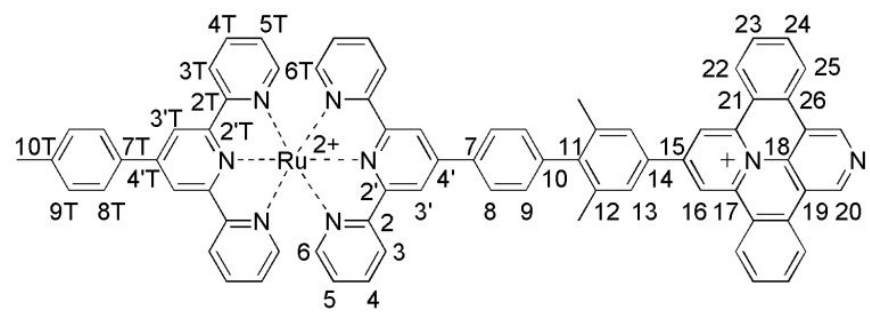

Dyad 2: [(ttpy)Ru(tpy-Ph-Xy-dBQNTH 2$)]\left(\mathrm{PF}_{6}\right)_{3}$

A solution of [4'-( $p$-tolyl)-2,2':6',2"-terpyridine]ruthenium trichloride (154 mg, $0.29 \mathrm{mmol})$ and 9-(4'-([2,2':6',2"'-terpyridin]-4'-yl)-2,6-dimethyl-[1,1'-biphenyl]-4-yl)-benzo[c] benzo[1,2]quinolizino[3,4,5,6-ija][1,6]naphthyridin-15-ium hexafluorophosphate ligand 2 (300 $\mathrm{mg}, 0.35$ $\mathrm{mmol})$ in anhydrous degassed ethylene glycol $(28 \mathrm{~mL})$ was sonicated for 2 min and then heated at $196{ }^{\circ} \mathrm{C}$ in a microwave oven $(\mathrm{P}=500 \mathrm{~W})$ for $10 \mathrm{~min}$ whilst stirring. The solution became brown. Absolute EtOH $(20 \mathrm{~mL}), \mathrm{NH}_{4} \mathrm{PF}_{6}(1.0 \mathrm{~g})$ and $\mathrm{H}_{2} \mathrm{O}(50 \mathrm{~mL})$ were added to the reaction mixture which was then stirred for $10 \mathrm{~min}$. The resulting red solid was filtered off and washed with $\mathrm{H}_{2} \mathrm{O}$ to remove solvents and excess salts. The solid was dissolved in acetone $(150 \mathrm{~mL})$ and EtOH $(50 \mathrm{~mL})$ was added. Acetone was removed under reduced pressure and the product precipitated in $\mathrm{EtOH}$. The precipitate was filtered off and washed with $\mathrm{Et}_{2} \mathrm{O}$. This solid was purified by column chromatography over basic alumina $\left(\mathrm{CH}_{2} \mathrm{Cl}_{2} /\right.$ acetone, $100 / 0$ to $0 / 100)$ to yield a red solid, which was reprecipitated in a mixture of acetone/ $\mathrm{Et}_{2} \mathrm{O}(294 \mathrm{mg})$. The resulting mixture was purified again by column chromatography over basic alumina $\left(\mathrm{CH}_{2} \mathrm{Cl}_{2}\right.$ /acetone, $100 / 0$ to $0 / 100$ and acetone $/ \mathrm{CH}_{3} \mathrm{CN}, 100 / 0$ to $\left.75 / 25\right)$ to yield a red solid (140 mg), which was recrystallized ten times by slow diffusion of $\mathrm{Et}_{2} \mathrm{O}$ in a concentrated $\mathrm{CH}_{3} \mathrm{CN}$ solution of the complex $(128 \mathrm{mg}, 28 \%) .{ }^{1} \mathrm{H}$ NMR $\left(500 \mathrm{MHz}, \mathrm{CD}_{3} \mathrm{CN}\right): \delta 10.26$ (s, $\left.2 \mathrm{H} ; \mathrm{H}^{20}\right) ; 9.66\left(\mathrm{~s}, 2 \mathrm{H} ; \mathrm{H}^{16}\right) ; 9.23\left(\mathrm{~d}, 2 \mathrm{H}, J=9.0 \mathrm{~Hz} ; \mathrm{H}^{22}\right) ; 9.12\left(\mathrm{~s}, 2 \mathrm{H} ; \mathrm{H}^{3^{\prime}}\right) ; 9.03-9.00(\mathrm{~m}, 4 \mathrm{H}$; $\left.\mathrm{H}^{25}, \mathrm{H}^{3} \mathrm{~T}\right) ; 8.71\left(\mathrm{~d}, 2 \mathrm{H}, J=8.0 \mathrm{~Hz} ; \mathrm{H}^{3}\right) ; 8.66\left(\mathrm{~d}, 2 \mathrm{H}, J=8.0 \mathrm{~Hz} ; \mathrm{H}^{3 \mathrm{~T}}\right) ; 8.42(\mathrm{~d}, 2 \mathrm{H}, J=8.0 \mathrm{~Hz}$; $\left.\mathrm{H}^{8}\right) ; 8.28\left(\mathrm{~s}, 2 \mathrm{H} ; \mathrm{H}^{13}\right) ; 8.23\left(\mathrm{t}, 2 \mathrm{H}, J=8.0 \mathrm{~Hz} ; \mathrm{H}^{24}\right) ; 8.13-8.11\left(\mathrm{~m}, 4 \mathrm{H} ; \mathrm{H}^{8 \mathrm{~T}, 23}\right) ; 8.00-7.94(\mathrm{~m}$, $\left.4 \mathrm{H} ; \mathrm{H}^{4}, \mathrm{H}^{4 \mathrm{~T}}\right) ; 7.69\left(\mathrm{~d}, 2 \mathrm{H}, J=8.0 \mathrm{~Hz} ; \mathrm{H}^{9}\right) ; 7.60\left(\mathrm{~d}, 2 \mathrm{H}, J=8.0 \mathrm{~Hz} ; \mathrm{H}^{9 \mathrm{~T}}\right) ; 7.47-7.46(\mathrm{~m}, 4 \mathrm{H}$; $\left.\mathrm{H}^{6,6 \mathrm{~T}}\right) ; 7.23-7.18\left(\mathrm{~m}, 4 \mathrm{H} ; \mathrm{H}^{5}, \mathrm{H}^{5 \mathrm{~T}}\right) ; 2.55\left(\mathrm{~s}, 3 \mathrm{H} ; \mathrm{H}^{\mathrm{Me}(10 \mathrm{~T})}\right) ; 2.40\left(\mathrm{~s}, 6 \mathrm{H} ; \mathrm{H}^{\operatorname{Me}(12)}\right) .{ }^{13} \mathrm{C} \mathrm{NMR}$ $\left(126 \mathrm{MHz}, \mathrm{CD}_{3} \mathrm{CN}\right): \delta 159.5\left(\mathrm{C}^{2 \mathrm{~T}}\right) ; 159.3\left(\mathrm{C}^{2}\right) ; 156.7\left(\mathrm{C}^{2}\right) ; 156.6\left(\mathrm{C}^{2} \mathrm{~T}\right) ; 153.6\left(\mathrm{C}^{6,6 \mathrm{~T}}\right) ; 152.1$ $\left(\mathrm{C}^{15}\right) ; 149.9\left(\mathrm{C}^{4} \mathrm{~T}\right) ; 148.8\left(\mathrm{C}^{4}\right) ; 146.4\left(\mathrm{C}^{20}\right) ; 146.3\left(\mathrm{C}^{11}\right) ; 144.9\left(\mathrm{C}^{17}\right) ; 143.2\left(\mathrm{C}^{10}\right) ; 142.2$ $\left(\mathrm{C}^{10 \mathrm{~T}}\right) ; 139.3\left(\mathrm{C}^{4,4 \mathrm{~T}}\right) ; 139.2\left(\mathrm{C}^{12}\right) ; 137.1\left(\mathrm{C}^{7}\right) ; 135.8\left(\mathrm{C}^{24}\right) ; 135.0\left(\mathrm{C}^{7 \mathrm{~T}}\right) ; 134.5\left(\mathrm{C}^{14}\right) ; 133.5$ $\left(\mathrm{C}^{18}\right) ; 132.4\left(\mathrm{C}^{23}\right) ; 131.4\left(\mathrm{C}^{9 \mathrm{~T}}\right) ; 131.2\left(\mathrm{C}^{9}\right) ; 129.2\left(\mathrm{C}^{8}\right) ; 129.1\left(\mathrm{C}^{13}\right) ; 128.8\left(\mathrm{C}^{8 \mathrm{~T}}\right) ; 128.6\left(\mathrm{C}^{5,5 \mathrm{~T}}\right)$; 
$128.4\left(\mathrm{C}^{26,22}\right) ; 125.9\left(\mathrm{C}^{21}\right) ; 125.7\left(\mathrm{C}^{3,3 \mathrm{~T}}\right) ; 124.4\left(\mathrm{C}^{25}\right) ; 122.6\left(\mathrm{C}^{3}\right) ; 122.5\left(\mathrm{C}^{3 \top} \mathrm{T}\right) ; 120.0\left(\mathrm{C}^{16}\right)$; $119.4\left(\mathrm{C}^{19}\right) ; 21.3\left(\mathrm{C}^{\mathrm{Me}(10 \mathrm{~T})}\right)$; $21.1\left(\mathrm{C}^{\mathrm{Me}(12)}\right)$. FTMS: $m / z$ : calcd for $\mathrm{C}_{73} \mathrm{H}_{51} \mathrm{~N}_{8} \mathrm{Ru}: 380.4428$ $\left[\mathrm{M}-3 \mathrm{PF}_{6}\right]^{3+}$; found: $380.4431\left[\mathrm{M}-3 \mathrm{PF}_{6}\right]^{3+}$.

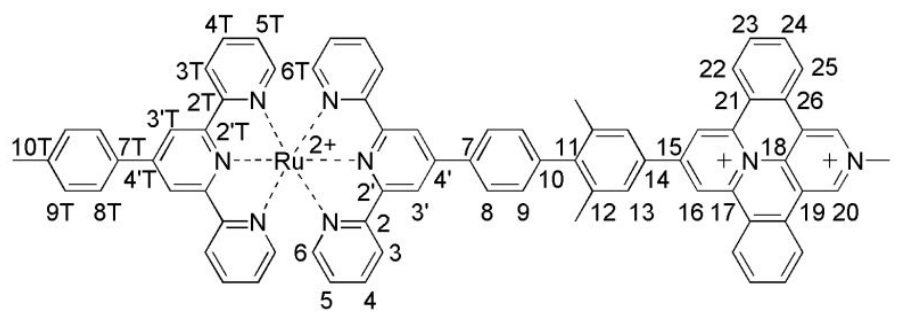

2: [(ttpy)Ru(tpy-Ph-Xy-MedBQNTH 2$)]\left(\mathrm{PF}_{6}\right)_{4}$

To a solution of dyad $2(24 \mathrm{mg}, 0.015 \mathrm{mmol})$ in $\mathrm{MeCN}(30 \mathrm{~mL})$ was added MeOTf $(0.3 \mathrm{~mL}$, $2.73 \mathrm{mmol})$ under argon. The reaction crude was then slowly transferred into a stirred aqueous solution of $\mathrm{KPF}_{6}(6.0 \mathrm{~g}$ in $150 \mathrm{~mL})$. The red precipitate of the $\mathrm{PF}_{6}{ }^{-}$complex was filtered off and washed with $\mathrm{H}_{2} \mathrm{O}$ and $\mathrm{Et}_{2} \mathrm{O}$. The solid is a mixture of the starting material (20\% molar) and the expected product ( $80 \%$ molar). This precipitate was dissolved another time in $\mathrm{CH}_{3} \mathrm{CN}(20 \mathrm{~mL})$ and flushed with argon before addition of MeOTf $(0.3 \mathrm{~mL}, 2.73$ mmol). The resulting solution was stirred for 20 min under argon. The reaction crude was then slowly transferred into a stirred aqueous solution of $\mathrm{KPF}_{6}(6.0 \mathrm{~g}$ in $150 \mathrm{~mL})$. The red precipitate of the $\mathrm{PF}_{6}{ }^{-}$complex was filtered off and washed with $\mathrm{H}_{2} \mathrm{O}$ and $\mathrm{Et}_{2} \mathrm{O}$, yielding the pure product as a red solid $(15 \mathrm{mg}, 57.6 \%) .{ }^{1} \mathrm{H}$ NMR $\left(500 \mathrm{MHz}, \mathrm{CD}_{3} \mathrm{CN}\right): \delta 10.16(\mathrm{~s}, 2 \mathrm{H}$; $\left.\mathrm{H}^{20}\right) ; 9.82\left(\mathrm{~s}, 2 \mathrm{H} ; \mathrm{H}^{16}\right) ; 9.37$ (d, $\left.2 \mathrm{H}, J=8.5 \mathrm{~Hz} ; \mathrm{H}^{22}\right) ; 9.12$ (s, $\left.2 \mathrm{H} ; \mathrm{H}^{3}\right) ; 9.02\left(\mathrm{~s}, 2 \mathrm{H} ; \mathrm{H}^{3} \mathrm{~T}\right)$; $8.91\left(\mathrm{~d}, 2 \mathrm{H}, J=8.0 \mathrm{~Hz} ; \mathrm{H}^{25}\right) ; 8.71\left(\mathrm{~d}, 2 \mathrm{H}, J=8.5 \mathrm{~Hz} ; \mathrm{H}^{3}\right) ; 8.66\left(\mathrm{~d}, 2 \mathrm{H}, J=8.0 \mathrm{~Hz} ; \mathrm{H}^{3 \mathrm{~T}}\right) ; 8.43$ $\left(\mathrm{d}, 2 \mathrm{H}, J=8.0 \mathrm{~Hz} ; \mathrm{H}^{8}\right) ; 8.39-8.36\left(\mathrm{~m}, 4 \mathrm{H} ; \mathrm{H}^{24}, \mathrm{H}^{13}\right) ; 8.32\left(\mathrm{t}, 2 \mathrm{H}, J=7.5 \mathrm{~Hz} ; \mathrm{H}^{23}\right) ; 8.12(\mathrm{~d}$, $\left.2 \mathrm{H}, J=7.5 \mathrm{~Hz} ; \mathrm{H}^{8 \mathrm{~T}}\right) ; 7.99-7.95\left(\mathrm{~m}, 4 \mathrm{H} ; \mathrm{H}^{4}, \mathrm{H}^{4 \mathrm{~T}}\right) ; 7.69\left(\mathrm{~d}, 2 \mathrm{H}, J=8.5 \mathrm{~Hz} ; \mathrm{H}^{9}\right) ; 7.60(\mathrm{~d}, 2 \mathrm{H}, J$ $\left.=8.0 \mathrm{~Hz} ; \mathrm{H}^{9 \mathrm{~T}}\right) ; 7.47-7.46\left(\mathrm{~m}, 4 \mathrm{H} ; \mathrm{H}^{6}, \mathrm{H}^{6 \mathrm{~T}}\right) ; 7.23-7.18\left(\mathrm{~m}, 4 \mathrm{H} ; \mathrm{H}^{5}, \mathrm{H}^{5 \mathrm{~T}}\right) ; 4.86\left(\mathrm{~s}, 3 \mathrm{H} ; \mathrm{H}^{\mathrm{Me}(\mathrm{N})}\right)$; $2.55\left(\mathrm{~s}, 3 \mathrm{H} ; \mathrm{H}^{\mathrm{Me}(10 \mathrm{~T})}\right) ; 2.42\left(\mathrm{~s}, 6 \mathrm{H} ; \mathrm{H}^{\mathrm{Me}(12)}\right) .{ }^{13} \mathrm{C} \mathrm{NMR}\left(126 \mathrm{MHz}, \mathrm{CD}_{3} \mathrm{CN}\right): \delta=159.1\left(\mathrm{C}^{2,2 \mathrm{~T}}\right)$; $156.6\left(\mathrm{C}^{2}\right) ; 156.3\left(\mathrm{C}^{2} \mathrm{~T}\right)$; $155.0\left(\mathrm{C}^{15}\right) ; 153.5\left(\mathrm{C}^{6,6 \mathrm{~T}}\right)$; $149.5\left(\mathrm{C}^{4} \mathrm{~T}\right) ; 148.4\left(\mathrm{C}^{4}\right) ; 147.2\left(\mathrm{C}^{11}\right)$; $145.0\left(\mathrm{C}^{17}\right) ; 142.8\left(\mathrm{C}^{10}\right) ; 142.0\left(\mathrm{C}^{10 \mathrm{~T}}\right) ; 141.3\left(\mathrm{C}^{20}\right) ; 139.3\left(\mathrm{C}^{12}\right) ; 139.1\left(\mathrm{C}^{4,4 \mathrm{~T}}\right) ; 137.0\left(\mathrm{C}^{7}\right)$; $136.6\left(\mathrm{C}^{24}\right) ; 135.5\left(\mathrm{C}^{18}\right) ; 134.8\left(\mathrm{C}^{7 \mathrm{~T}}\right) ; 134.7\left(\mathrm{C}^{23}\right)$; $133.8\left(\mathrm{C}^{14}\right) ; 131.3\left(\mathrm{C}^{9 \mathrm{~T}}\right) ; 131.1\left(\mathrm{C}^{9}\right) ; 129.4$ $\left(\mathrm{C}^{13}\right) ; 129.2\left(\mathrm{C}^{8}\right) ; 128.9\left(\mathrm{C}^{22}\right) ; 128.7\left(\mathrm{C}^{8 \mathrm{~T}}\right) ; 128.5\left(\mathrm{C}^{5,5 \mathrm{~T}}\right) ; 126.5\left(\mathrm{C}^{21}\right) ; 125.7\left(\mathrm{C}^{26}\right) ; 125.5\left(\mathrm{C}^{3}\right.$, $\left.\mathrm{C}^{3 \mathrm{~T}}\right)$; $124.9\left(\mathrm{C}^{25}\right) ; 124.6\left(\mathrm{C}^{19}\right) ; 122.5\left(\mathrm{C}^{3}\right) ; 122.4\left(\mathrm{C}^{3} \mathrm{~T}\right) ; 121.0\left(\mathrm{C}^{16}\right) ; 50.7\left(\mathrm{C}^{\mathrm{Me}(\mathrm{N})}\right) ; 21.3$ $\left(\mathrm{C}^{\mathrm{Me}(10 \mathrm{~T})}\right) ; \quad 21.2 \quad\left(\mathrm{C}^{\mathrm{Me}(12)}\right)$. FTMS: $m / z$ : calcd for $\mathrm{C}_{75} \mathrm{H}_{57} \mathrm{O}_{3} \mathrm{~N}_{8} \mathrm{Ru}: \quad 406.4527$ $\left[\mathrm{M}-4 \mathrm{PF}_{6}+\mathrm{CH}_{3} \mathrm{O}^{-}+\mathrm{O}_{2}\right]^{3+}$; found: $406.4533\left[\mathrm{M}-4 \mathrm{PF}_{6}+\mathrm{CH}_{3} \mathrm{O}^{-}+\mathrm{O}_{2}\right]^{3+}$.

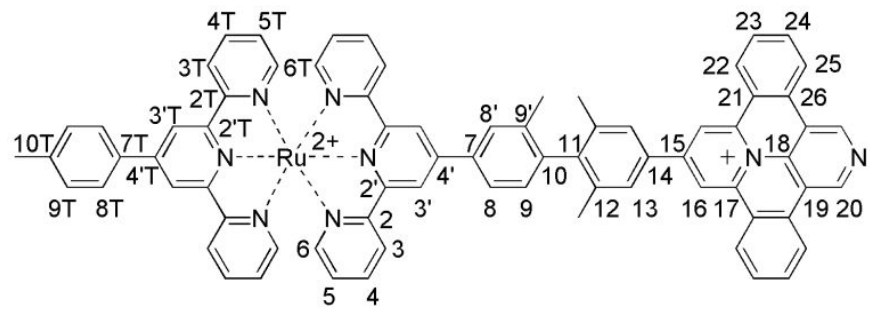

Dyad 3: [(ttpy)Ru(tpy-Tol-Xy-dBQNTH 2$)]\left(\mathrm{PF}_{6}\right)_{3}$

A solution of $\left[4^{\prime}-(p\right.$-tolyl $)-2,2^{\prime}: 6^{\prime}, 2^{\prime \prime}$-terpyridine]ruthenium trichloride $(216 \mathrm{mg}, 0.41 \mathrm{mmol})$ and 9-(4'-([2,2':6',2"-terpyridin]-4'-yl)-2,2',6-trimethyl-[1,1'-biphenyl]-4-yl)-benzo[c]benzo[1,2] quinolizino[3,4,5,6-ija][1,6]naphthyridin-15-ium tetrafluoroborate ligand 3 (400 mg, $0.49 \mathrm{mmol})$ in anhydrous degassed ethylene glycol $(40 \mathrm{~mL})$ was sonicated for 2 min and then heated at $196{ }^{\circ} \mathrm{C}$ in a microwave oven $(\mathrm{P}=500 \mathrm{~W})$ for $10 \mathrm{~min}$ whilst stirring. The solution became brown. Absolute EtOH $(20 \mathrm{~mL}), \mathrm{NH}_{4} \mathrm{PF}_{6}(1.0 \mathrm{~g})$ and $\mathrm{H}_{2} \mathrm{O}(50 \mathrm{~mL})$ were added to the reaction mixture which was then stirred for $10 \mathrm{~min}$. The resulting red solid was filtered off 
and washed with $\mathrm{H}_{2} \mathrm{O}$ to remove solvents and excess salts. The solid was dissolved in acetone $(150 \mathrm{~mL})$ and $\mathrm{EtOH}(50 \mathrm{~mL})$ was added. Acetone was removed under reduced pressure and the product precipitated in $\mathrm{EtOH}$. The precipitate was filtered off and washed with $\mathrm{Et}_{2} \mathrm{O}$. The solid was purified by column chromatography over basic alumina $\left(\mathrm{CH}_{2} \mathrm{Cl}_{2} /\right.$ acetone, $100 / 0$ to $0 / 100$ ) to yield a red solid, which was reprecipitated in a mixture of acetone $/ \mathrm{Et}_{2} \mathrm{O}$. The resulting solid was purified again by column chromatography over basic alumina $\left(\mathrm{CH}_{2} \mathrm{Cl}_{2}\right.$ /acetone, $100 / 0$ to $0 / 100$ and acetone $/ \mathrm{CH}_{3} \mathrm{CN}, 100 / 0$ to $\left.75 / 25\right)$ to yield a red solid $\left(143 \mathrm{mg}\right.$ ), which was recrystallized ten times by slow diffusion of $\mathrm{Et}_{2} \mathrm{O}$ in a concentrated $\mathrm{CH}_{3} \mathrm{CN}$ solution of the complex $(105 \mathrm{mg}, 16 \%) .{ }^{1} \mathrm{H}$ NMR $\left(500 \mathrm{MHz}, \mathrm{CD}_{3} \mathrm{CN}\right): \delta 10.26(\mathrm{~s}$, $\left.2 \mathrm{H} ; \mathrm{H}^{20}\right) ; 9.66\left(\mathrm{~s}, 2 \mathrm{H} ; \mathrm{H}^{16}\right) ; 9.23\left(\mathrm{~d}, 2 \mathrm{H}, J=9.0 \mathrm{~Hz} ; \mathrm{H}^{22}\right) ; 9.12\left(\mathrm{~s}, 2 \mathrm{H} ; \mathrm{H}^{3}\right) ; 9.02-9.00(\mathrm{~m}, 4 \mathrm{H}$; $\left.\mathrm{H}^{25}, \mathrm{H}^{3} \mathrm{~T}\right) ; 8.72\left(\mathrm{~d}, 2 \mathrm{H}, J=8.0 \mathrm{~Hz} ; \mathrm{H}^{3}\right) ; 8.66\left(\mathrm{~d}, 2 \mathrm{H}, J=7.5 \mathrm{~Hz} ; \mathrm{H}^{3 \mathrm{~T}}\right) ; 8.34\left(\mathrm{~s}, 1 \mathrm{H} ; \mathrm{H}^{8^{\prime}}\right) ; 8.30$ (s, 2H; $\left.\mathrm{H}^{13}\right) ; 8.26-8.21\left(\mathrm{~m}, 3 \mathrm{H} ; \mathrm{H}^{8}, \mathrm{H}^{24}\right) ; 8.14-8.11\left(\mathrm{~m}, 4 \mathrm{H} ; \mathrm{H}^{8 \mathrm{~T}}, \mathrm{H}^{23}\right) ; 7.98(\mathrm{t}, 2 \mathrm{H}, J=8.0$ $\left.\mathrm{Hz} ; \mathrm{H}^{4}\right) ; 7.97\left(\mathrm{t}, 2 \mathrm{H}, J=8.0 \mathrm{~Hz} ; \mathrm{H}^{4 \mathrm{~T}}\right) ; 7.60\left(\mathrm{~d}, 2 \mathrm{H}, J=8.0 \mathrm{~Hz} ; \mathrm{H}^{9 \mathrm{~T}}\right) ; 7.53(\mathrm{~d}, 1 \mathrm{H}, J=8.0 \mathrm{~Hz}$; $\left.\mathrm{H}^{9}\right) ; 7.48-7.45$ (m, 4H; $\left.\mathrm{H}^{6}, \mathrm{H}^{6 \mathrm{~T}}\right) ; 7.23-7.19\left(\mathrm{~m}, 4 \mathrm{H} ; \mathrm{H}^{5}, \mathrm{H}^{5 \mathrm{~T}}\right) ; 2.55$ (s, 3H; $\left.\mathrm{H}^{\mathrm{Me}(10 \mathrm{~T})}\right) ; 2.33$ (s, $\left.3 \mathrm{H} ; \mathrm{H}^{\mathrm{Me}\left(9^{\prime}\right)}\right) ; 2.32\left(\mathrm{~s}, 6 \mathrm{H} ; \mathrm{H}^{\mathrm{Me}(12)}\right) .{ }^{13} \mathrm{C} \mathrm{NMR}\left(126 \mathrm{MHz}, \mathrm{CD}_{3} \mathrm{CN}\right): \delta 159.2\left(\mathrm{C}^{2,2 \mathrm{~T}}\right) ; 156.6\left(\mathrm{C}^{2^{\prime}}\right)$; $156.4\left(\mathrm{C}^{2} \mathrm{~T}\right) ; 153.4\left(\mathrm{C}^{6,6 \mathrm{~T}}\right) ; 152.8\left(\mathrm{C}^{15}\right) ; 149.4\left(\mathrm{C}^{4} \mathrm{~T}\right) ; 148.7\left(\mathrm{C}^{4}\right) ; 146.2\left(\mathrm{C}^{20}\right) ; 145.6\left(\mathrm{C}^{11}\right)$; $144.8\left(\mathrm{C}^{17}\right) ; 142.7\left(\mathrm{C}^{10}\right) ; 142.1\left(\mathrm{C}^{10 \mathrm{~T}}\right) ; 139.0\left(\mathrm{C}^{4,4 \mathrm{~T}}\right) ; 138.9\left(\mathrm{C}^{12}\right) ; 138.2\left(\mathrm{C}^{9}\right) ; 137.1\left(\mathrm{C}^{7}\right)$; $135.6\left(\mathrm{C}^{24}\right)$; $134.9\left(\mathrm{C}^{7 \mathrm{~T}}\right)$; $134.4\left(\mathrm{C}^{14}\right) ; 133.3\left(\mathrm{C}^{18}\right) ; 132.3\left(\mathrm{C}^{23}\right) ; 131.3\left(\mathrm{C}^{9 \mathrm{~T}}\right) ; 130.8\left(\mathrm{C}^{9}\right) ; 130.6$ $\left(\mathrm{C}^{8}\right) ; 128.9\left(\mathrm{C}^{13}\right) ; 128.6\left(\mathrm{C}^{8 \mathrm{~T}}\right) ; 128.4\left(\mathrm{C}^{5,5 \mathrm{~T}}\right) ; 128.3\left(\mathrm{C}^{26,22}\right) ; 126.7\left(\mathrm{C}^{8}\right) ; 126.6\left(\mathrm{C}^{21}\right) ; 125.5$ $\left(\mathrm{C}^{3,3 \mathrm{~T}}\right) ; 124.2\left(\mathrm{C}^{25}\right) ; 122.3\left(\mathrm{C}^{3^{\prime}, 3^{3} \mathrm{~T}}\right) ; 119.8\left(\mathrm{C}^{16}\right) ; 119.3\left(\mathrm{C}^{19}\right) ; 21.2\left(\mathrm{C}^{\operatorname{Me}(10 \mathrm{~T})}\right) ; 20.6\left(\mathrm{C}^{\operatorname{Me}(12)}\right)$; $19.8\left(\mathrm{C}^{\mathrm{Me}\left(9^{\prime}\right)}\right)$. FTMS: $m / z$ : calcd for $\mathrm{C}_{74} \mathrm{H}_{53} \mathrm{~N}_{8} \mathrm{Ru}: 385.1149\left[\mathrm{M}-3 \mathrm{PF}_{6}\right]^{3+}$; found: 385.1147 $\left[\mathrm{M}-3 \mathrm{PF}_{6}\right]^{3+}$.

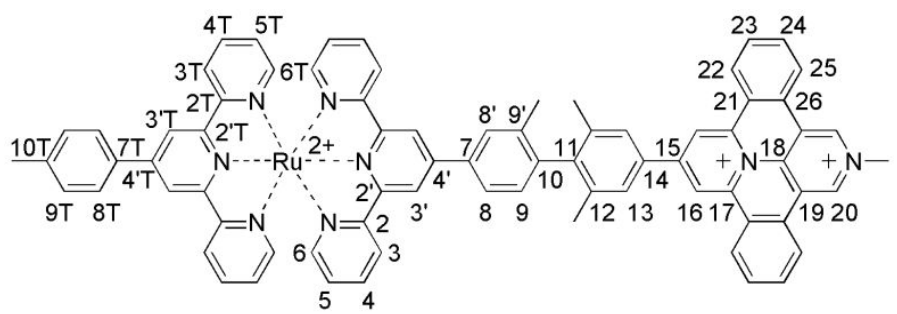

3: $[($ ttpy $) \mathrm{Ru}($ tpy-Tol-Xy-MedBQNTH2 $)]\left(\mathrm{PF}_{6}\right)_{4}$

To a solution of dyad $3(31 \mathrm{mg}, 0.020 \mathrm{mmol})$ in MeCN $(25 \mathrm{~mL})$ was added MeOTf $(0.414$ $\mathrm{mL}, 3.77 \mathrm{mmol}$ ) under argon. The mixture was stirred for $20 \mathrm{~min}$ under argon. The reaction crude was then slowly transferred into a stirred aqueous solution of $\mathrm{KPF}_{6}(6.0 \mathrm{~g}$ in $150 \mathrm{~mL})$. The red precipitate of the $\mathrm{PF}_{6}{ }^{-}$complex was filtered off and washed with $\mathrm{H}_{2} \mathrm{O}$ and $\mathrm{Et}_{2} \mathrm{O}$. The solid is a mixture of the starting material ( $34 \%$ molar) and the expected product ( $66 \%$ molar). This precipitate was dissolved another time in $\mathrm{CH}_{3} \mathrm{CN}(25 \mathrm{~mL})$ and flushed with argon before addition of MeOTf $(0.864 \mathrm{~mL}, 7.88 \mathrm{mmol})$. The resulting solution was stirred for $30 \mathrm{~min}$ under argon. The reaction crude was then slowly transferred into a stirred aqueous solution of $\mathrm{KPF}_{6}(6.0 \mathrm{~g}$ in $150 \mathrm{~mL})$. The red precipitate of the $\mathrm{PF}_{6}{ }^{-}$complex was filtered off and washed with $\mathrm{H}_{2} \mathrm{O}$ and $\mathrm{Et}_{2} \mathrm{O}$, yielding the pure product as a red solid $(23 \mathrm{mg}, 65.7 \%)$. ${ }^{1} \mathrm{H}$ NMR (500 $\left.\mathrm{MHz}, \mathrm{CD}_{3} \mathrm{CN}\right): \delta 10.16\left(\mathrm{~s}, 2 \mathrm{H} ; \mathrm{H}^{20}\right) ; 9.83\left(\mathrm{~s}, 2 \mathrm{H} ; \mathrm{H}^{16}\right) ; 9.37$ (d, $\left.2 \mathrm{H}, J=9.0 \mathrm{~Hz} ; \mathrm{H}^{22}\right) ; 9.12$ (s, $2 \mathrm{H} ; \mathrm{H}^{3}$ ) $; 9.02\left(\mathrm{~s}, 2 \mathrm{H} ; \mathrm{H}^{3} \mathrm{~T}\right) ; 8.91\left(\mathrm{~d}, 2 \mathrm{H}, J=8.0 \mathrm{~Hz} ; \mathrm{H}^{25}\right) ; 8.71\left(\mathrm{~d}, 2 \mathrm{H}, J=8.0 \mathrm{~Hz} ; \mathrm{H}^{3}\right) ; 8.66$ $\left(\mathrm{d}, 2 \mathrm{H}, J=8.0 \mathrm{~Hz} ; \mathrm{H}^{3 \mathrm{~T}}\right) ; 8.39\left(\mathrm{~s}, 2 \mathrm{H} ; \mathrm{H}^{13}\right) ; 8.38-8.30\left(\mathrm{~m}, 5 \mathrm{H} ; \mathrm{H}^{24}, \mathrm{H}^{8}, \mathrm{H}^{23}\right) ; 8.26(\mathrm{dd}, 1 \mathrm{H}, J=$ $\left.8.0 \mathrm{~Hz} ; \mathrm{H}^{8}\right) ; 8.12\left(\mathrm{~d}, 2 \mathrm{H}, J=7.5 \mathrm{~Hz} ; \mathrm{H}^{8 \mathrm{~T}}\right) ; 8.00-7.95\left(\mathrm{~m}, 4 \mathrm{H} ; \mathrm{H}^{4}, \mathrm{H}^{4 \mathrm{~T}}\right) ; 7.60(\mathrm{~d}, 2 \mathrm{H}, J=8.0$ $\left.\mathrm{Hz} ; \mathrm{H}^{9 \mathrm{~T}}\right) ; 7.53\left(\mathrm{~d}, 1 \mathrm{H}, J=7.5 \mathrm{~Hz} ; \mathrm{H}^{9}\right) ; 7.48-7.45\left(\mathrm{~m}, 4 \mathrm{H} ; \mathrm{H}^{6}, \mathrm{H}^{6 \mathrm{~T}}\right) ; 7.23-7.18\left(\mathrm{~m}, 4 \mathrm{H} ; \mathrm{H}^{5}\right.$, $\left.\mathrm{H}^{5 \mathrm{~T}}\right) ; 4.86\left(\mathrm{~s}, 3 \mathrm{H} ; \mathrm{H}^{\mathrm{Me}(\mathrm{N})}\right) ; 2.55\left(\mathrm{~s}, 3 \mathrm{H} ; \mathrm{H}^{\mathrm{Me}(10 \mathrm{~T})}\right) ; 2.34\left(\mathrm{~s}, 6 \mathrm{H} ; \mathrm{H}^{\operatorname{Me}(12)}\right) ; 2.33\left(\mathrm{~s}, 3 \mathrm{H} ; \mathrm{H}^{\mathrm{Me}\left(9^{\prime}\right)}\right)$. ${ }^{13} \mathrm{C}$ NMR $\left(126 \mathrm{MHz}, \mathrm{CD}_{3} \mathrm{CN}\right): \delta 159.3\left(\mathrm{C}^{2,2 \mathrm{~T}}\right) ; 156.6\left(\mathrm{C}^{2}\right) ; 156.4\left(\mathrm{C}^{2} \mathrm{~T}\right) ; 155.1\left(\mathrm{C}^{15}\right) ; 153.4$ $\left(\mathrm{C}^{6,6 \mathrm{~T}}\right) ; 149.4\left(\mathrm{C}^{4} \mathrm{~T}\right) ; 148.6\left(\mathrm{C}^{4}\right) ; 146.7\left(\mathrm{C}^{11}\right) ; 145.0\left(\mathrm{C}^{17}\right) ; 142.4\left(\mathrm{C}^{10}\right) ; 142.1\left(\mathrm{C}^{10 \mathrm{~T}}\right) ; 141.2$ 
$\left(\mathrm{C}^{20}\right) ; 139.2\left(\mathrm{C}^{12}\right) ; 139.0\left(\mathrm{C}^{4,4 \mathrm{~T}}\right) ; 138.1\left(\mathrm{C}^{9}\right) ; 137.2\left(\mathrm{C}^{7}\right) ; 136.5\left(\mathrm{C}^{24}\right) ; 135.5\left(\mathrm{C}^{18}\right) ; 134.8\left(\mathrm{C}^{7 \mathrm{~T}}\right)$; $134.6\left(\mathrm{C}^{23}\right)$; $133.8\left(\mathrm{C}^{14}\right) ; 131.3\left(\mathrm{C}^{9 \mathrm{~T}}\right) ; 130.7\left(\mathrm{C}^{9}\right) ; 130.6\left(\mathrm{C}^{8}\right) ; 129.4\left(\mathrm{C}^{13}\right) ; 128.9\left(\mathrm{C}^{22}\right) ; 128.6$ $\left(\mathrm{C}^{8 \mathrm{~T}}\right) ; 128.4\left(\mathrm{C}^{5,5 \mathrm{~T}}\right) ; 126.7\left(\mathrm{C}^{8}\right) ; 126.5\left(\mathrm{C}^{21}\right) ; 125.7\left(\mathrm{C}^{26}\right) ; 125.5\left(\mathrm{C}^{3,3 \mathrm{~T}}\right) ; 124.9\left(\mathrm{C}^{25}\right) ; 124.6$ $\left(\mathrm{C}^{19}\right) ; 122.3\left(\mathrm{C}^{3^{\prime}, 3^{\top} \mathrm{T}}\right) ; 120.9\left(\mathrm{C}^{16}\right) ; 50.7\left(\mathrm{C}^{\mathrm{Me}(\mathrm{N})}\right) ; 21.2\left(\mathrm{C}^{\mathrm{Me}(10 \mathrm{~T})}\right) ; 20.6\left(\mathrm{C}^{\mathrm{Me}(12)}\right) ; 19.8\left(\mathrm{C}^{\mathrm{Me}\left(9^{\prime}\right)}\right)$. FTMS: $m / z$ : calcd for $\mathrm{C}_{75} \mathrm{H}_{56} \mathrm{~N}_{8} \mathrm{Ru}: 292.5912\left[\mathrm{M}-4 \mathrm{PF}_{6}\right]^{4+}$; found: $292.5919\left[\mathrm{M}-4 \mathrm{PF}_{6}\right]^{4+}$.

\section{$\underline{\text { References }}$}

[S1] Crosby, G. A.; Demas, J. N. J. Phys. Chem. 1971, 75, 991

[S2] Nakamaru, N. Bull. Chem. Soc. Jpn. 1982, 55, 2697

[S3] Pavlishchuk, V. V.; Addison, A. W. Inorg. Chim. Acta 2000, 298, 97

[S4] Fortage, J.; Puntoriero, F.; Tuyèras, F.; Dupeyre, G.; Arrigo, A.; Ciofini, I.; Lainé, P. P.; Campagna, S. Inorg. Chem. 2012, 51, 5342-5352.

[S5] Fortage, J.; Peltier, C.; Nastasi, F.; Puntoriero, F.; Tuyèras, F.; Griveau, S.; Bedioui, F.; Adamo, C.; Ciofini, I.; Campagna, S.; Lainé, P. P. J. Am. Chem. Soc. 2010, 132, 1670016713.

[S6] Fortage, J.; Tuyèras, F.; Peltier, C.; Dupeyre, G.; Calboréan, A.; Bedioui, F.; Ochsenbein, P.; Puntoriero, F.; Campagna, S.; Ciofini, I.; Lainé, P. P. J. Phys. Chem. A 2012, 116, 7880-7891.

[S7] a) Collin, J.-P.; Guillerez, S.; Sauvage, J.-P.; Barigelletti, F.; De Cola, L.; Flamigni, L.; Balzani, V. Inorg. Chem. 1991, 30, 4230-4238. b) Collin, J.-P.; Guillerez, S.; Sauvage, J.-P. J. Chem. Soc., Chem. Commun. 1989, 776-778. c) Sullivan, B. P.; Calvert, J. M.; Meyer, T. J. Inorg. Chem. 1980, 19, 1404-1407. 


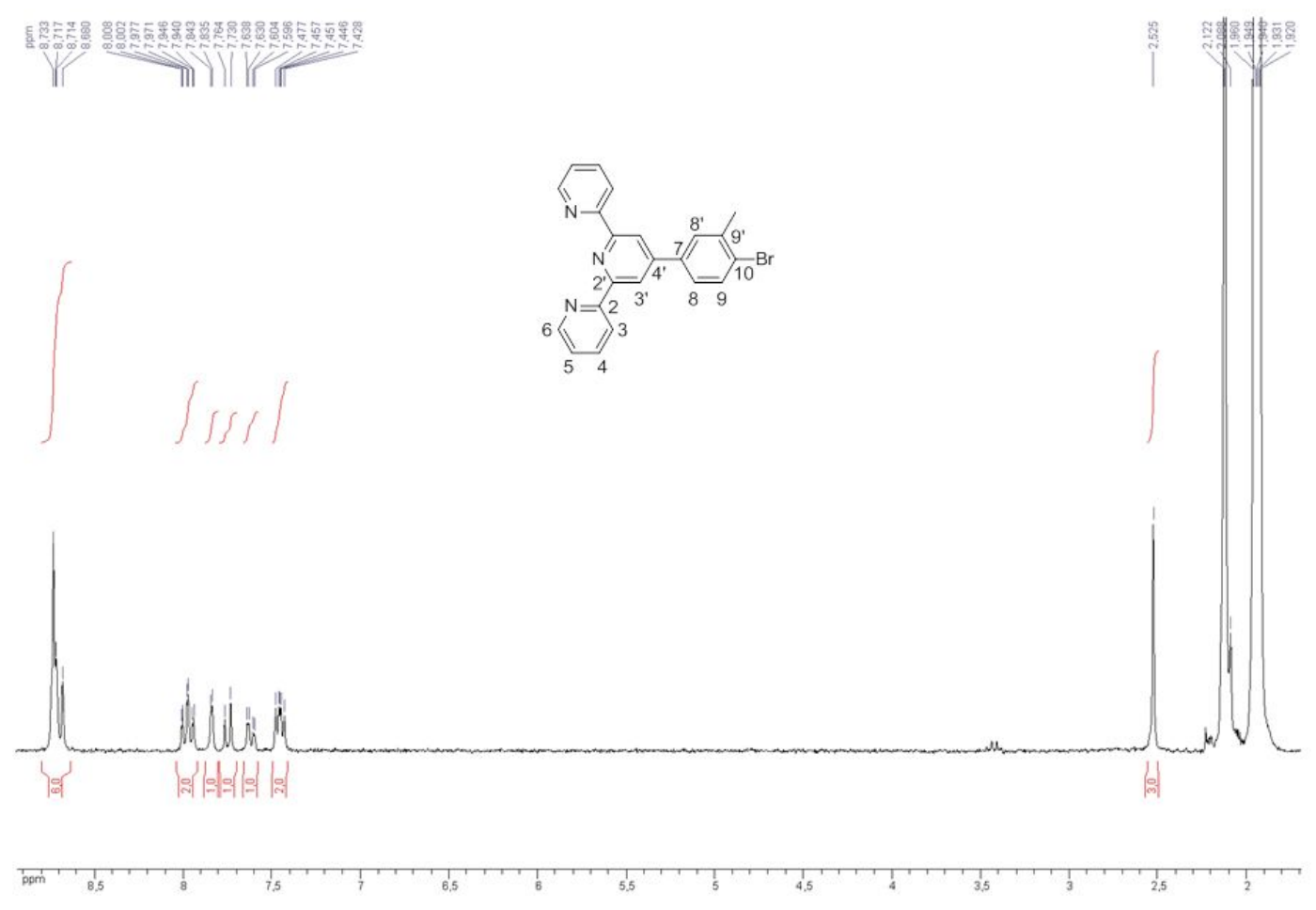

Figure S-3a. ' $\mathrm{H}$ NMR spectrum $(250 \mathrm{MHz})$ of 4'-(4-bromo-3-methylphenyl)-2,2':6',2"terpyridine in $\mathrm{CD}_{3} \mathrm{CN}$ (full spectrum).
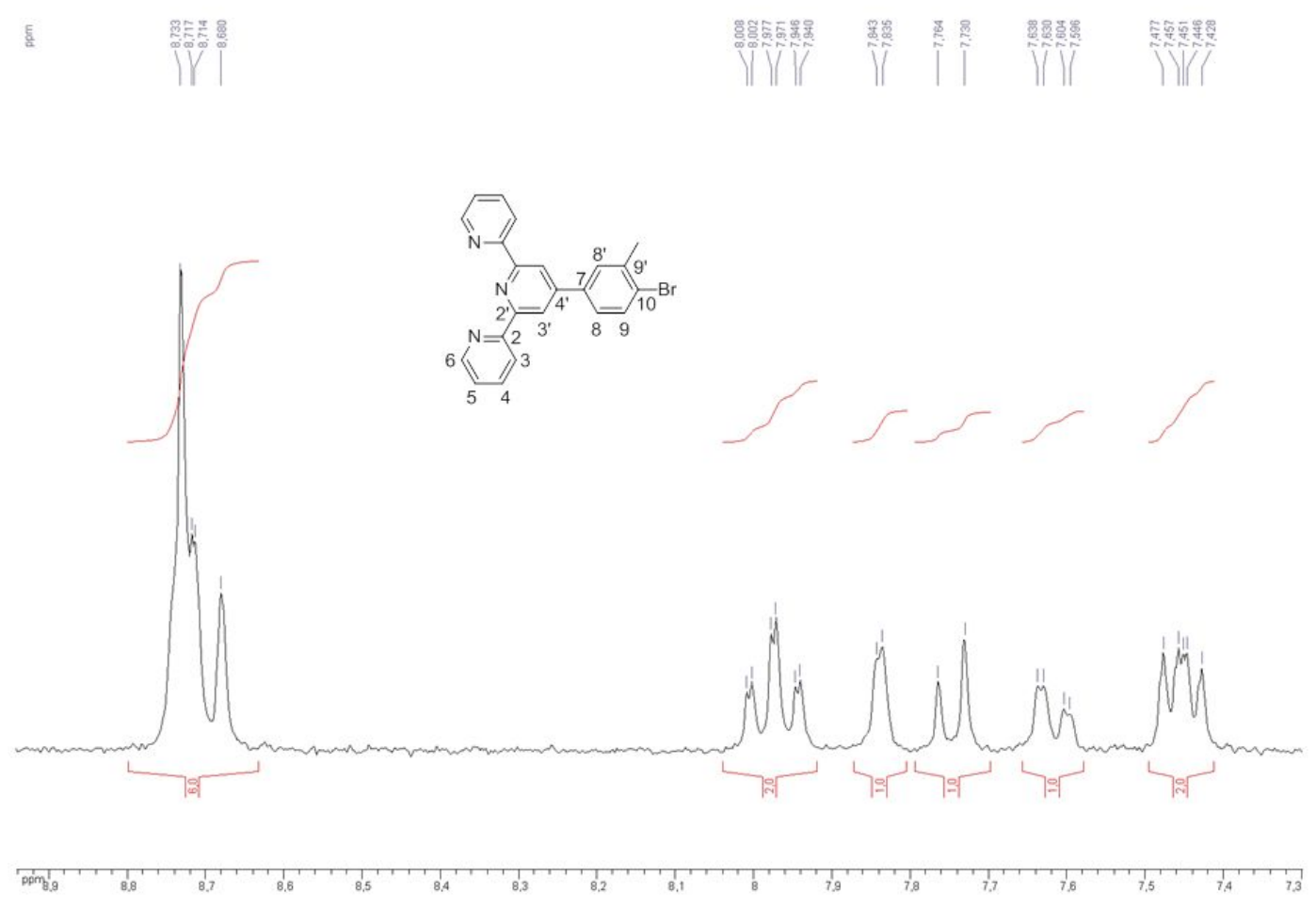

Figure S-3b. ${ }^{1} \mathrm{H}$ NMR spectrum (250 MHz) of 4'-(4-bromo-3-methylphenyl)-2,2':6',2"terpyridine in $\mathrm{CD}_{3} \mathrm{CN}$ (aromatic part). 

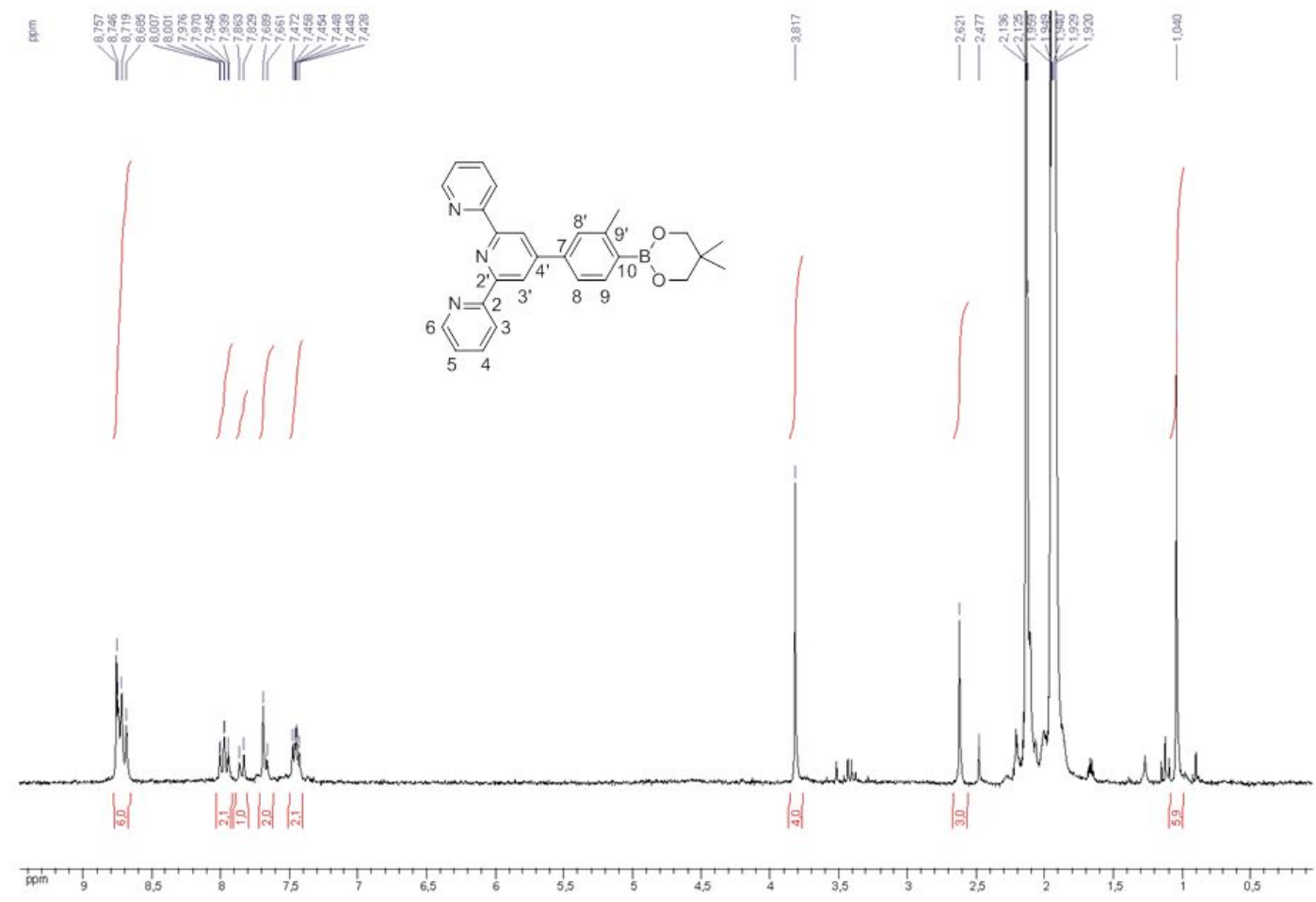

Figure S-4a. $\quad{ }^{1} \mathrm{H} \quad \mathrm{NMR} \quad$ spectrum $\quad(250 \quad \mathrm{MHz})$ of 4 '-[3-methyl-4(neopentylglycolatoboron)phenyl]-2,2':6',2"-terpyridine in $\mathrm{CD}_{3} \mathrm{CN}$ (full spectrum).

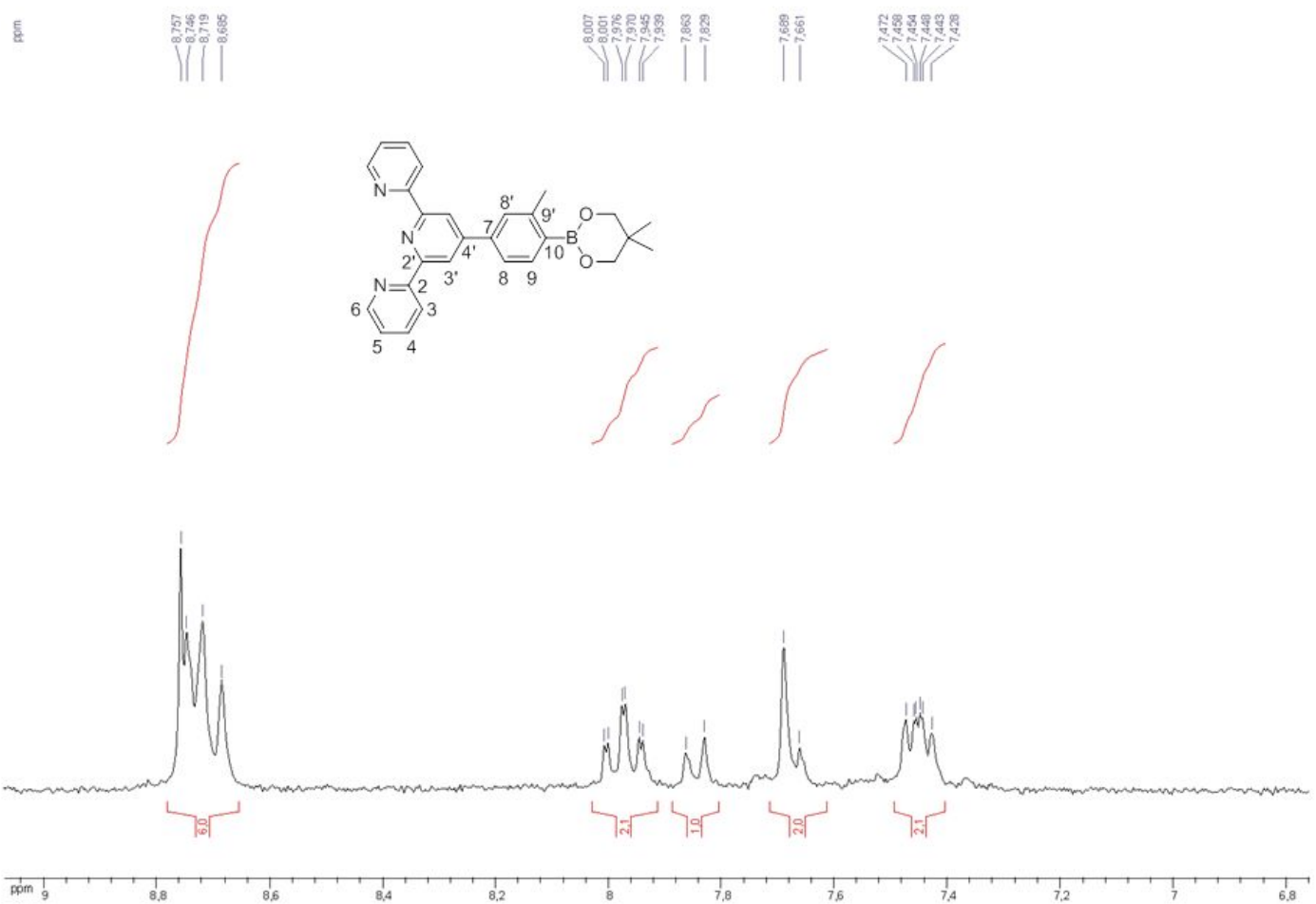

Figure S-4b. $\quad{ }^{1} \mathrm{H} \quad \mathrm{NMR} \quad$ spectrum $\quad(250 \quad \mathrm{MHz}) \quad$ of $\quad$ 4'-[3-methyl-4(neopentylglycolatoboron)phenyl]-2,2':6',2"-terpyridine in $\mathrm{CD}_{3} \mathrm{CN}$ (aromatic part). 


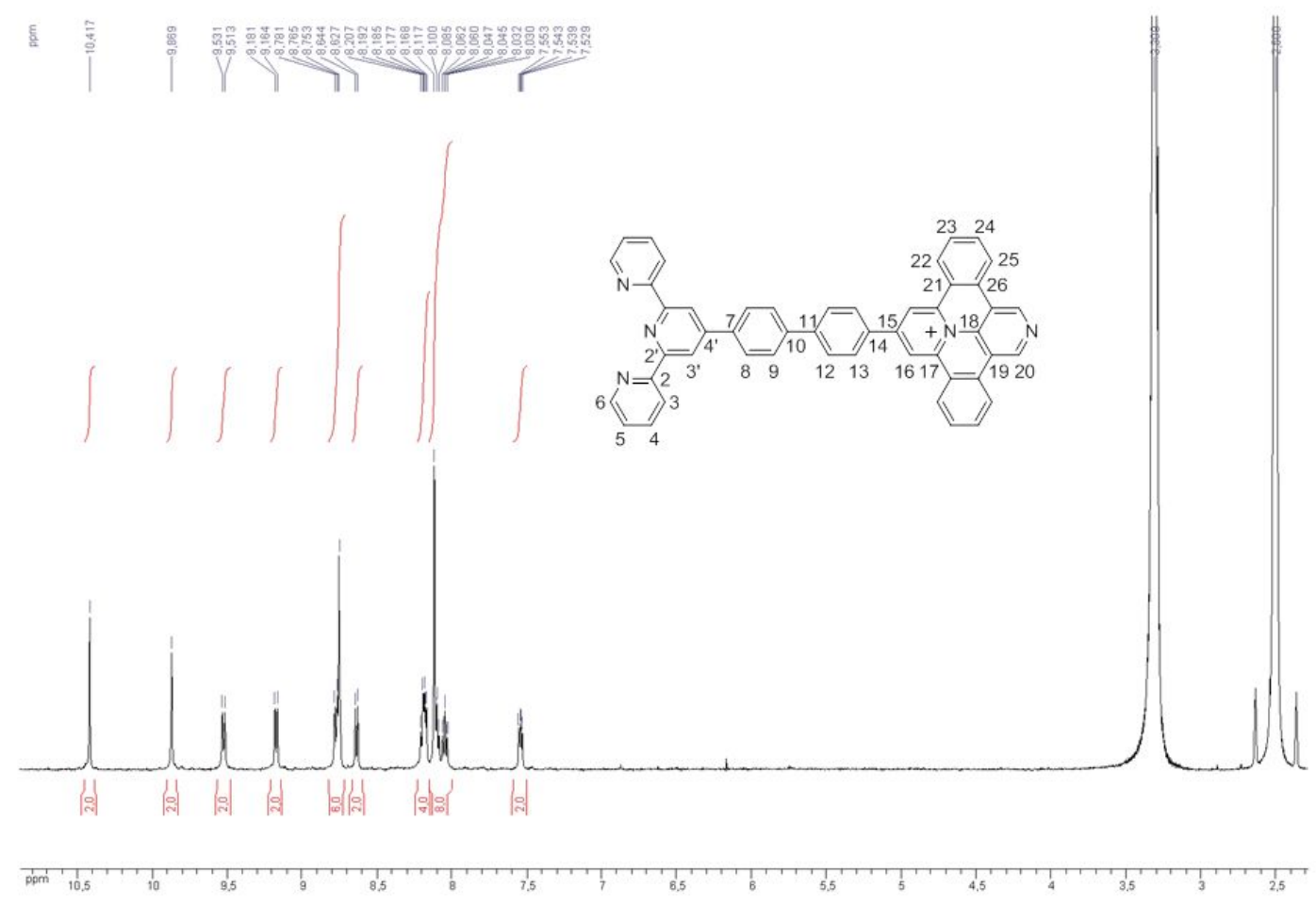

Figure S-5a. ${ }^{1} \mathrm{H}$ NMR spectrum $(500 \mathrm{MHz})$ of ligand 1 in DMSO- $d^{6}$ (full spectrum).

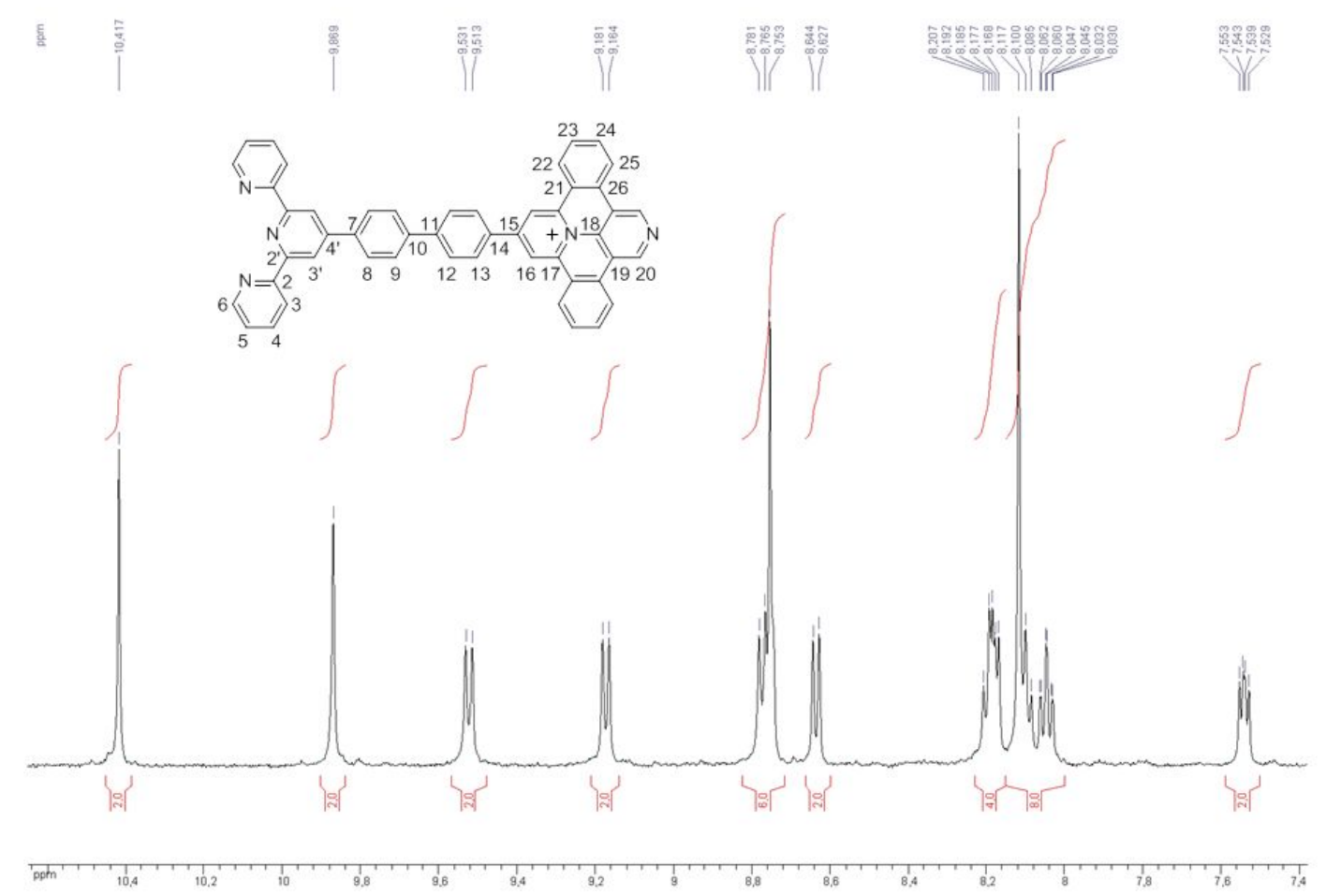

Figure S-5b. ${ }^{1} \mathrm{H}$ NMR spectrum $(500 \mathrm{MHz})$ of ligand 1 in DMSO- $d^{6}$ (aromatic part). 


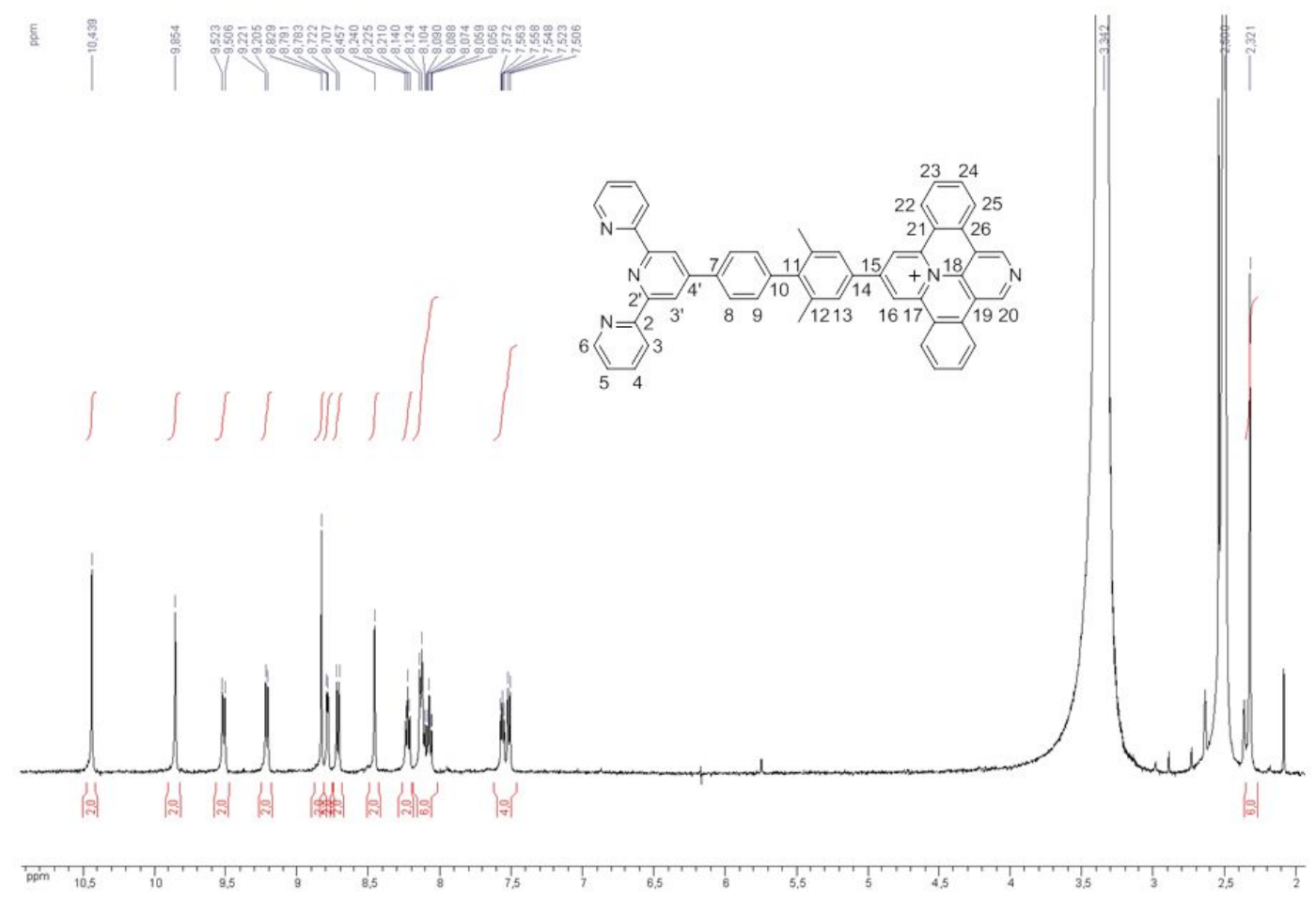

Figure S-6a. ${ }^{1} \mathrm{H}$ NMR spectrum $(500 \mathrm{MHz})$ of ligand 2 in DMSO- $d^{6}$ (full spectrum).

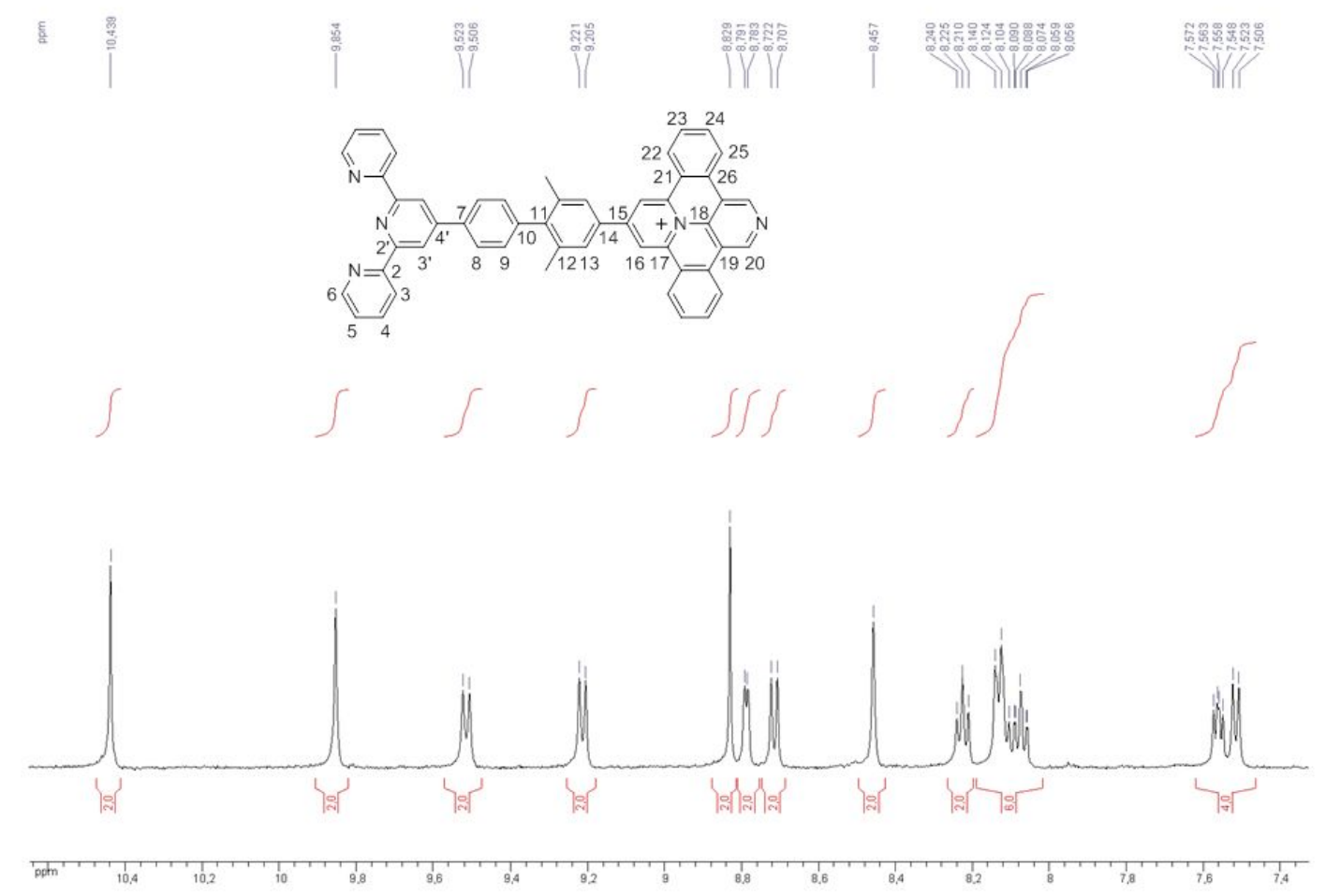

Figure S-6b. ${ }^{1} \mathrm{H}$ NMR spectrum $(500 \mathrm{MHz})$ of ligand 2 in DMSO- $d^{6}$ (aromatic part). 

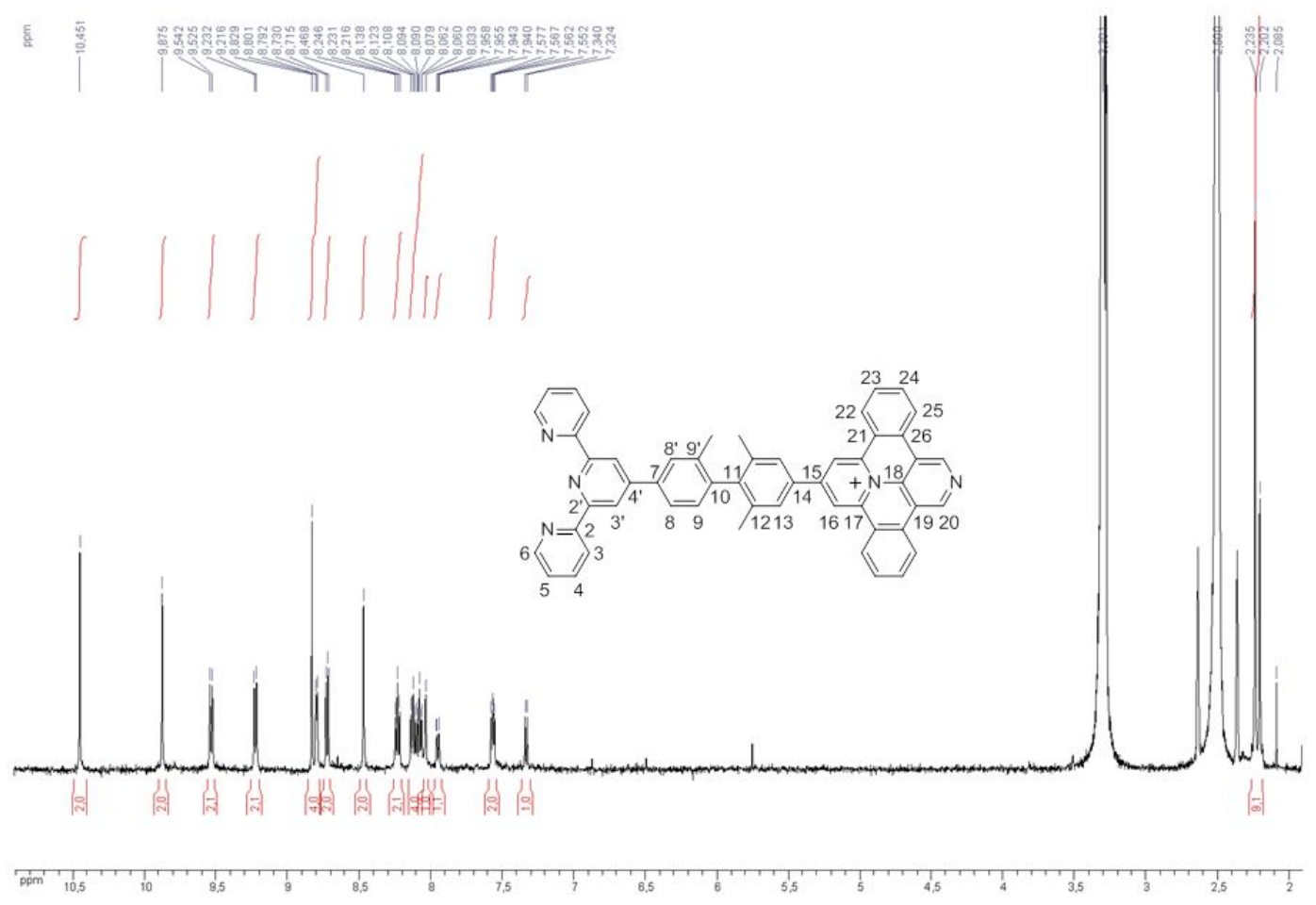

Figure S-7a. ${ }^{1} \mathrm{H}$ NMR spectrum $(500 \mathrm{MHz})$ of ligand 3 in DMSO- $d^{6}$ (full spectrum).

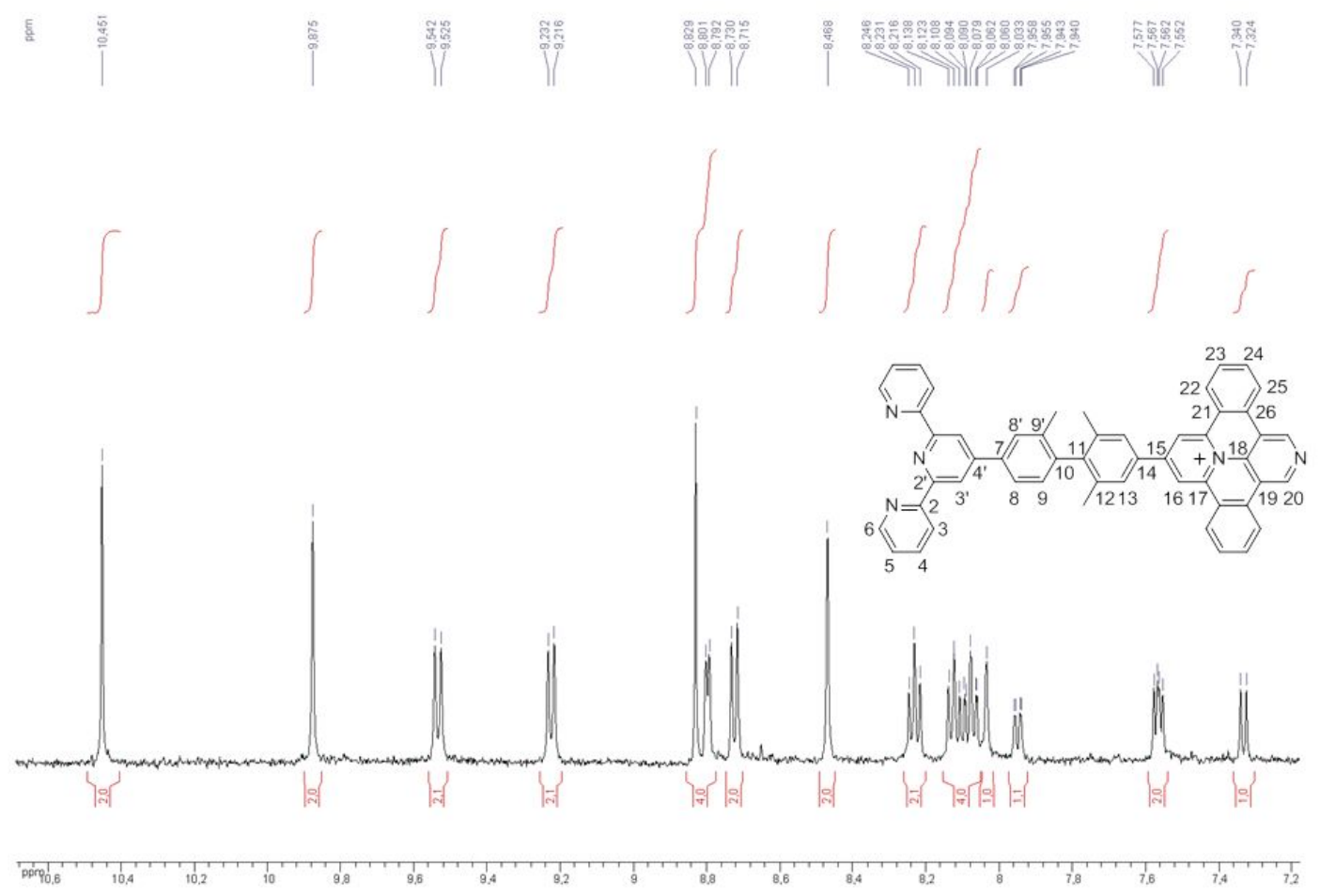

Figure S-7b. ${ }^{1} \mathrm{H}$ NMR spectrum $(500 \mathrm{MHz})$ of ligand 3 in DMSO- $d^{6}$ (aromatic part). 

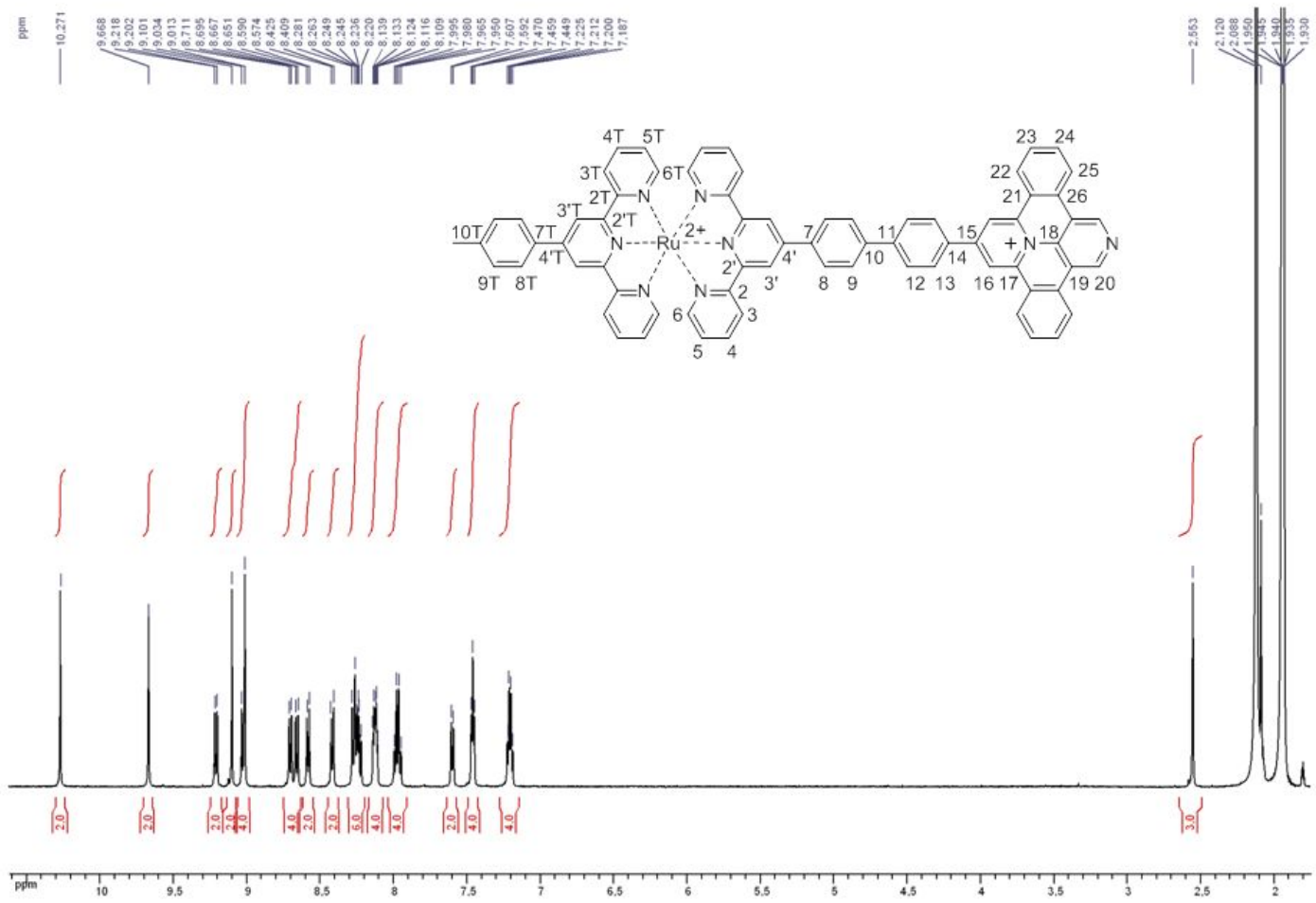

Figure S-8a. ${ }^{1} \mathrm{H}$ NMR spectrum $(500 \mathrm{MHz})$ of dyad 1 in $\mathrm{CD}_{3} \mathrm{CN}$ (full spectrum).

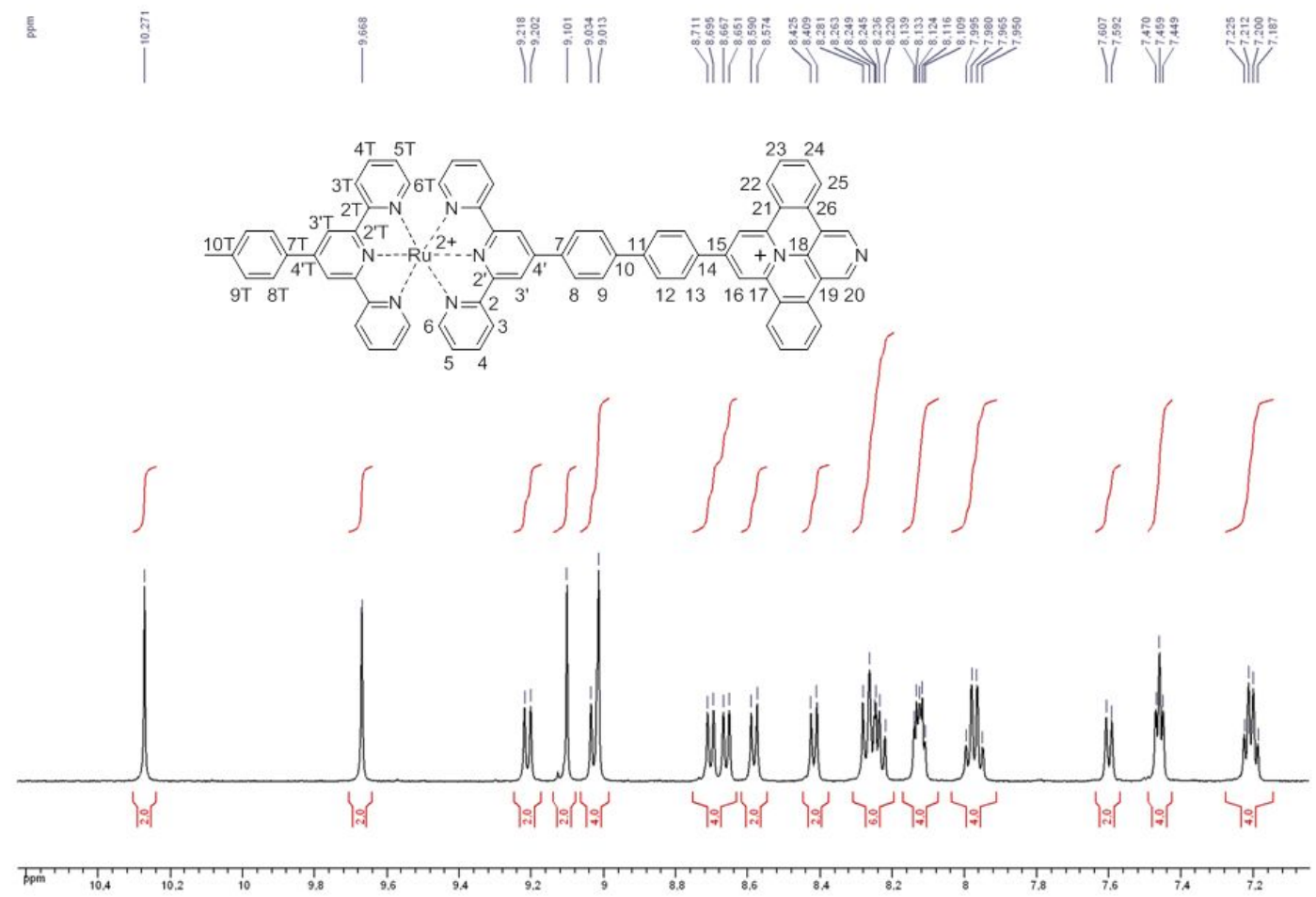

Figure S-8b. ${ }^{1} \mathrm{H}$ NMR spectrum $(500 \mathrm{MHz})$ of dyad 1 in $\mathrm{CD}_{3} \mathrm{CN}$ (aromatic part). 

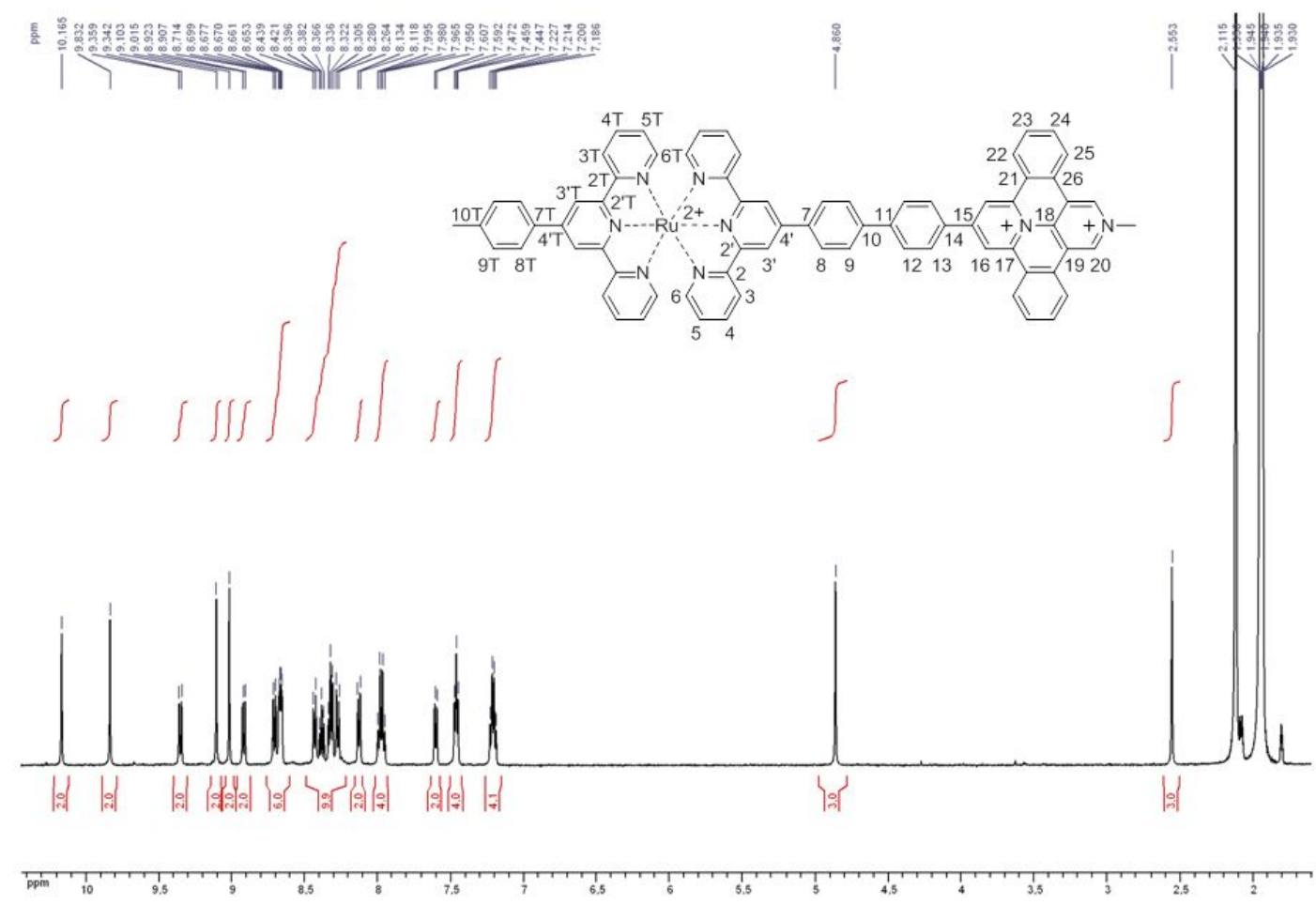

Figure S-9a. ${ }^{1} \mathrm{H}$ NMR spectrum $(500 \mathrm{MHz})$ of 1 in $\mathrm{CD}_{3} \mathrm{CN}$ (full spectrum).

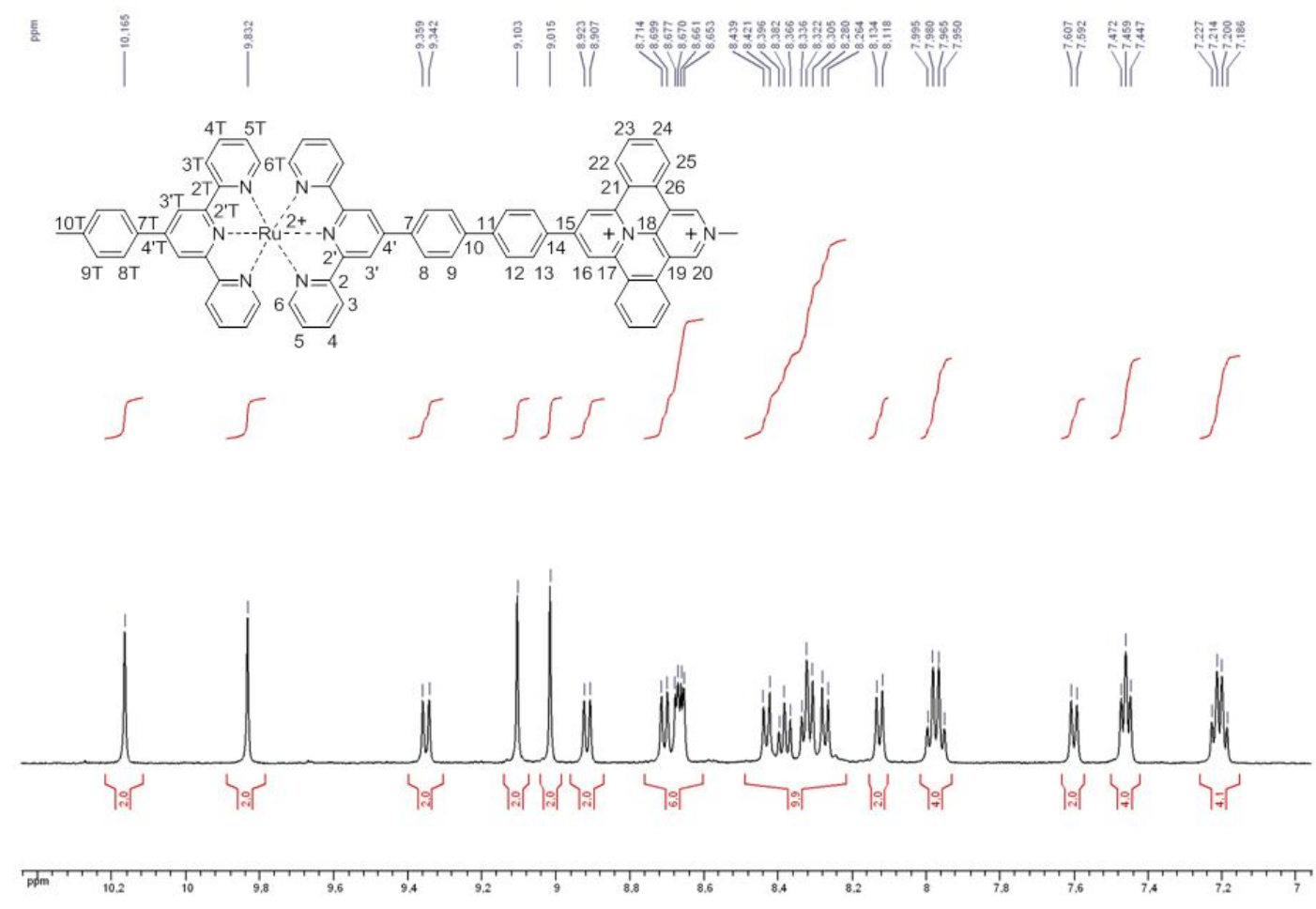

Figure S-9b. ${ }^{1} \mathrm{H}$ NMR spectrum $(500 \mathrm{MHz})$ of 1 in $\mathrm{CD}_{3} \mathrm{CN}$ (aromatic part). 

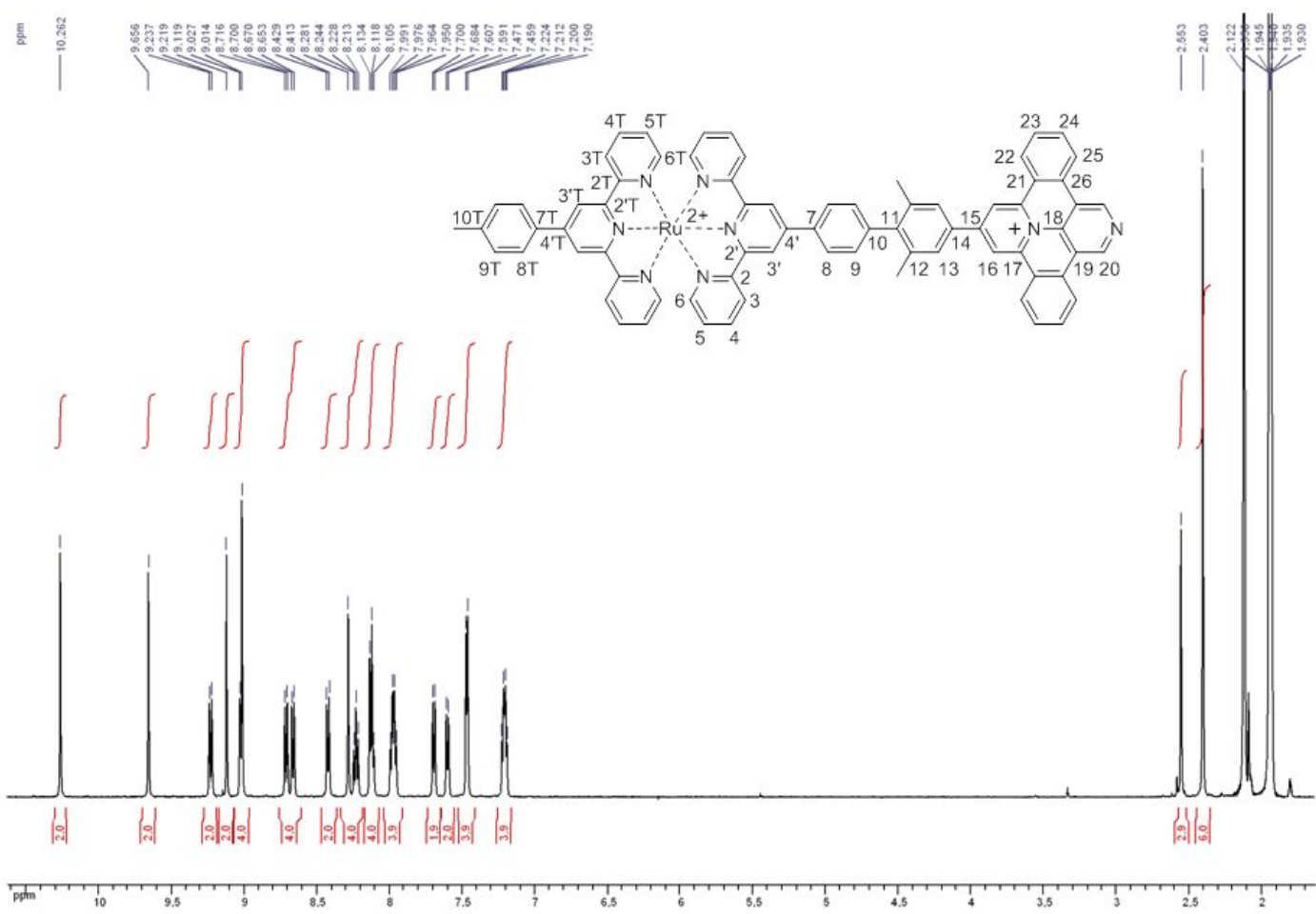

Figure S-10a. ${ }^{1} \mathrm{H}$ NMR spectrum $(500 \mathrm{MHz})$ of dyad 2 in $\mathrm{CD}_{3} \mathrm{CN}$ (full spectrum).
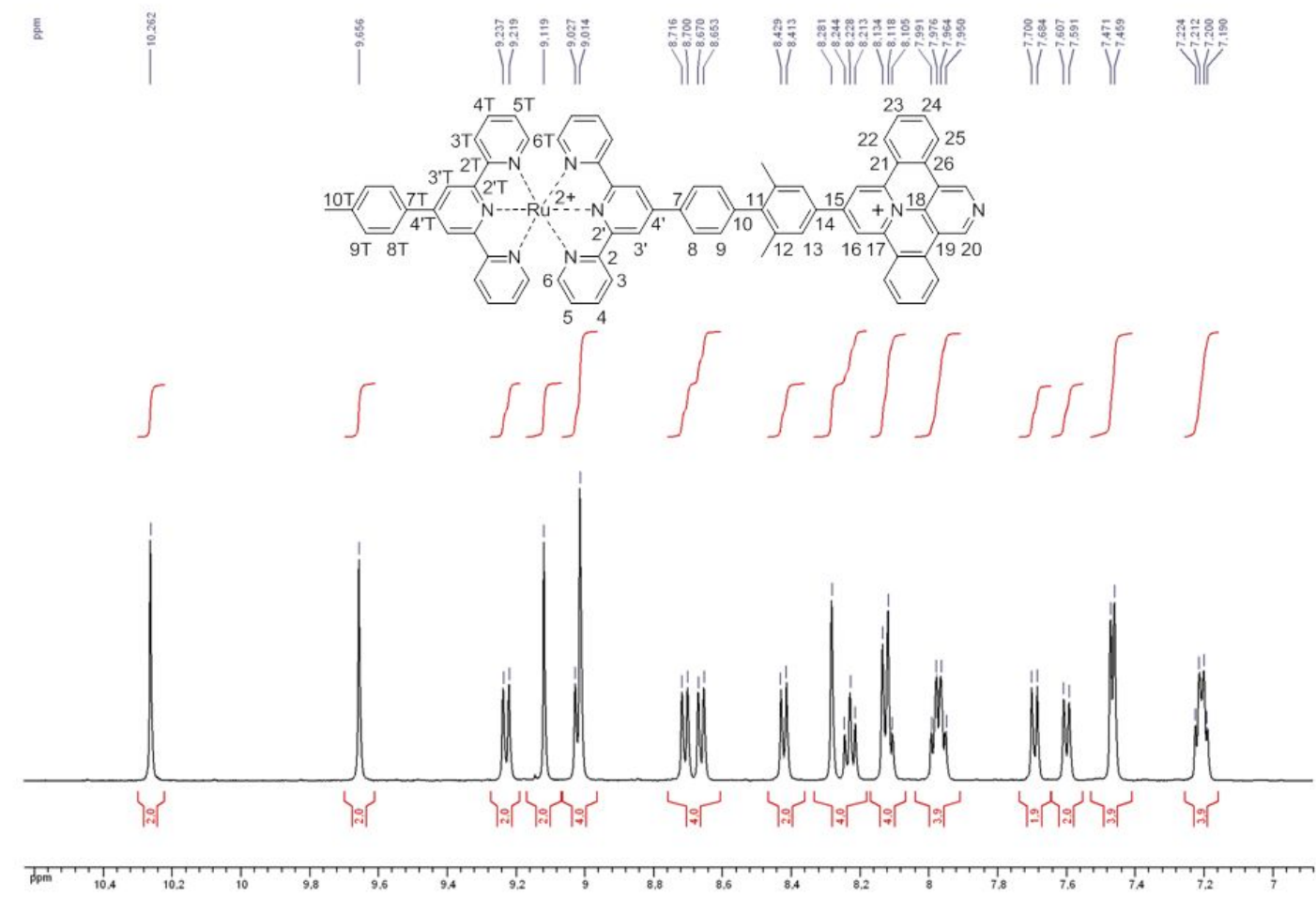

Figure S-10b. ${ }^{1} \mathrm{H}$ NMR spectrum $(500 \mathrm{MHz})$ of dyad 2 in $\mathrm{CD}_{3} \mathrm{CN}$ (aromatic part). 

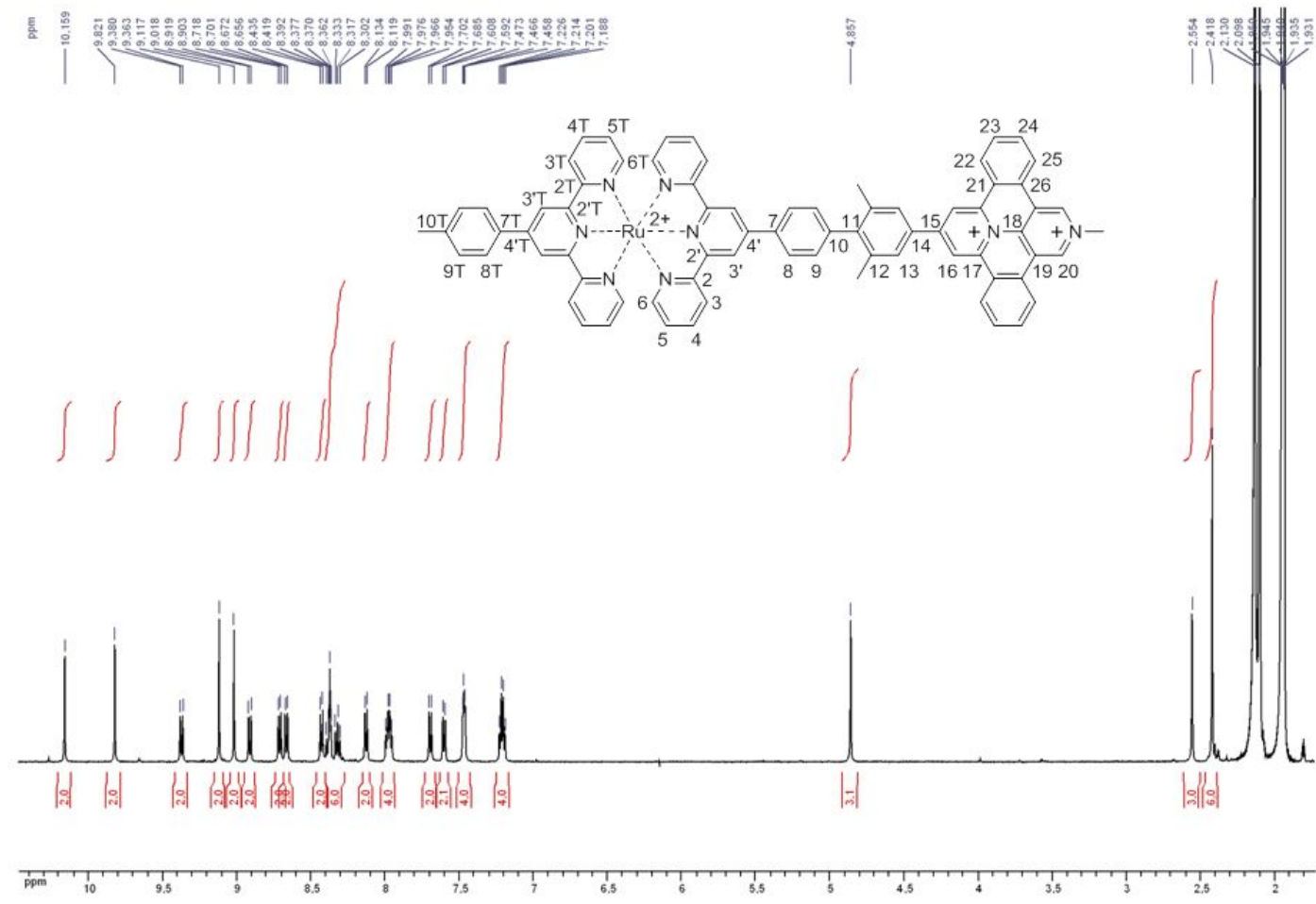

Figure S-11a. ${ }^{1} \mathrm{H}$ NMR spectrum $(500 \mathrm{MHz})$ of 2 in $\mathrm{CD}_{3} \mathrm{CN}$ (full spectrum).

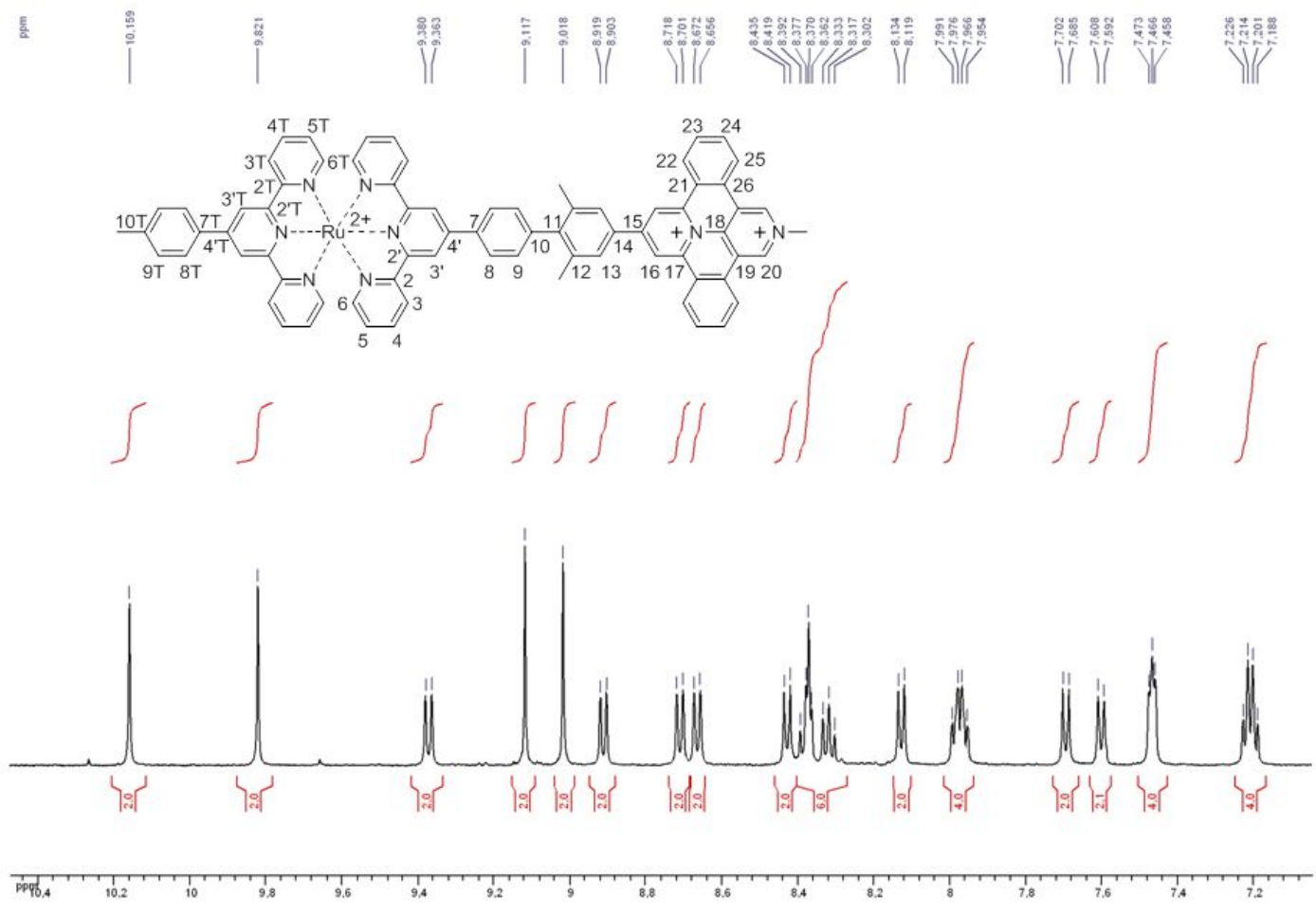

Figure S-11b. ${ }^{1} \mathrm{H}$ NMR spectrum $(500 \mathrm{MHz})$ of 2 in $\mathrm{CD}_{3} \mathrm{CN}$ (aromatic part). 

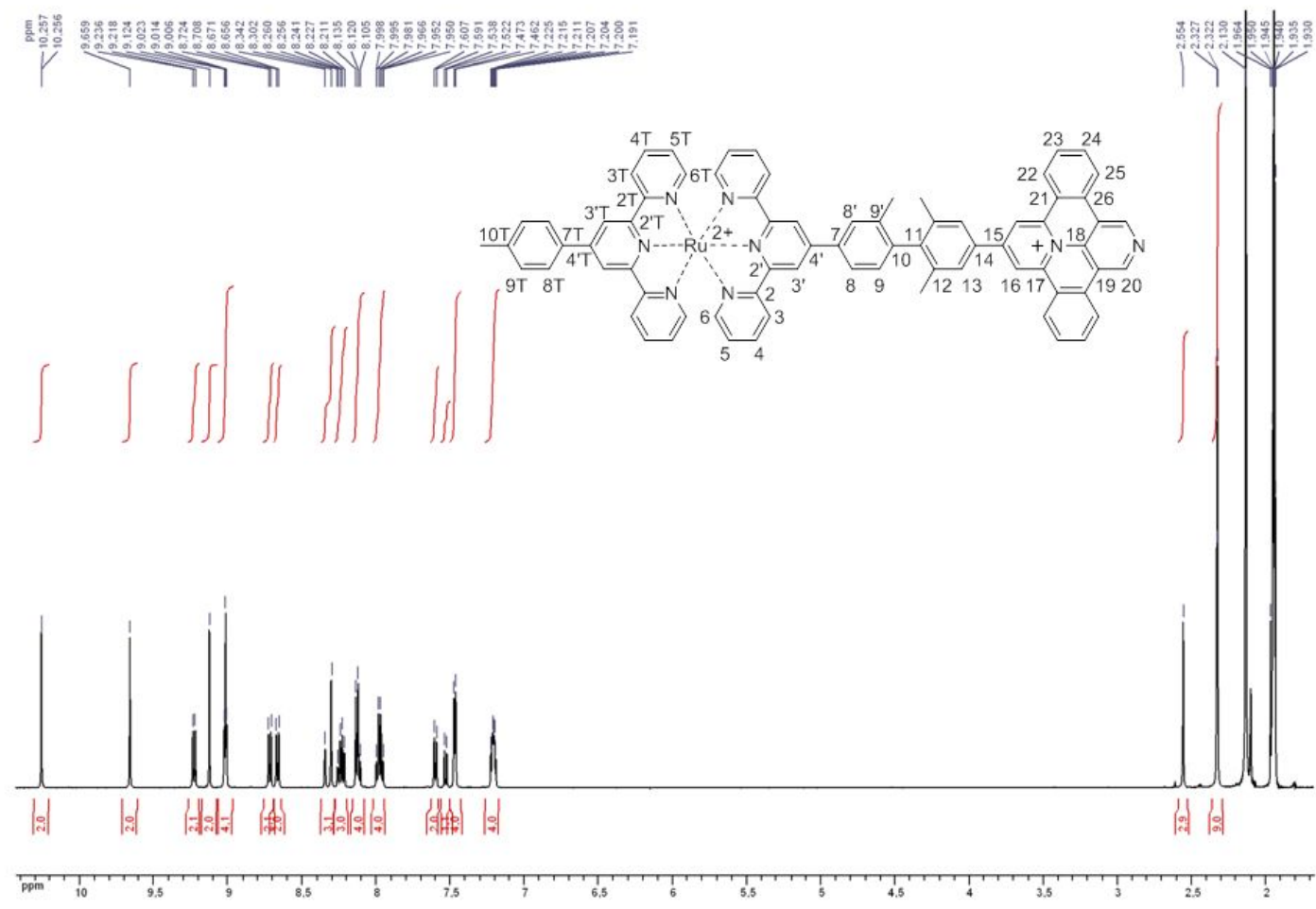

Figure S-12a. ${ }^{1} \mathrm{H}$ NMR spectrum $(500 \mathrm{MHz})$ of dyad 3 in $\mathrm{CD}_{3} \mathrm{CN}$ (full spectrum).

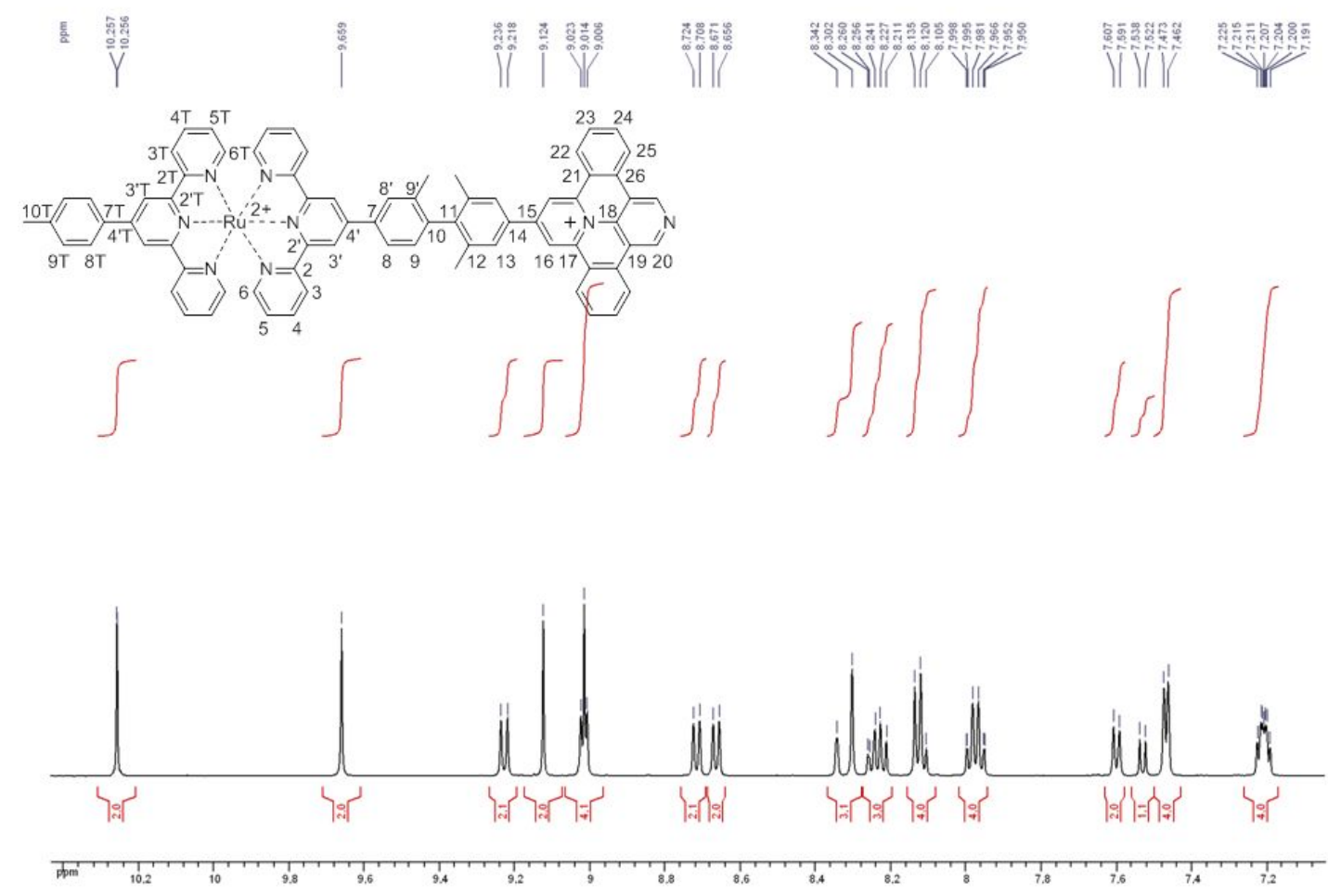

Figure S-12b. ${ }^{1} \mathrm{H}$ NMR spectrum $(500 \mathrm{MHz})$ of dyad 3 in $\mathrm{CD}_{3} \mathrm{CN}$ (aromatic part). 

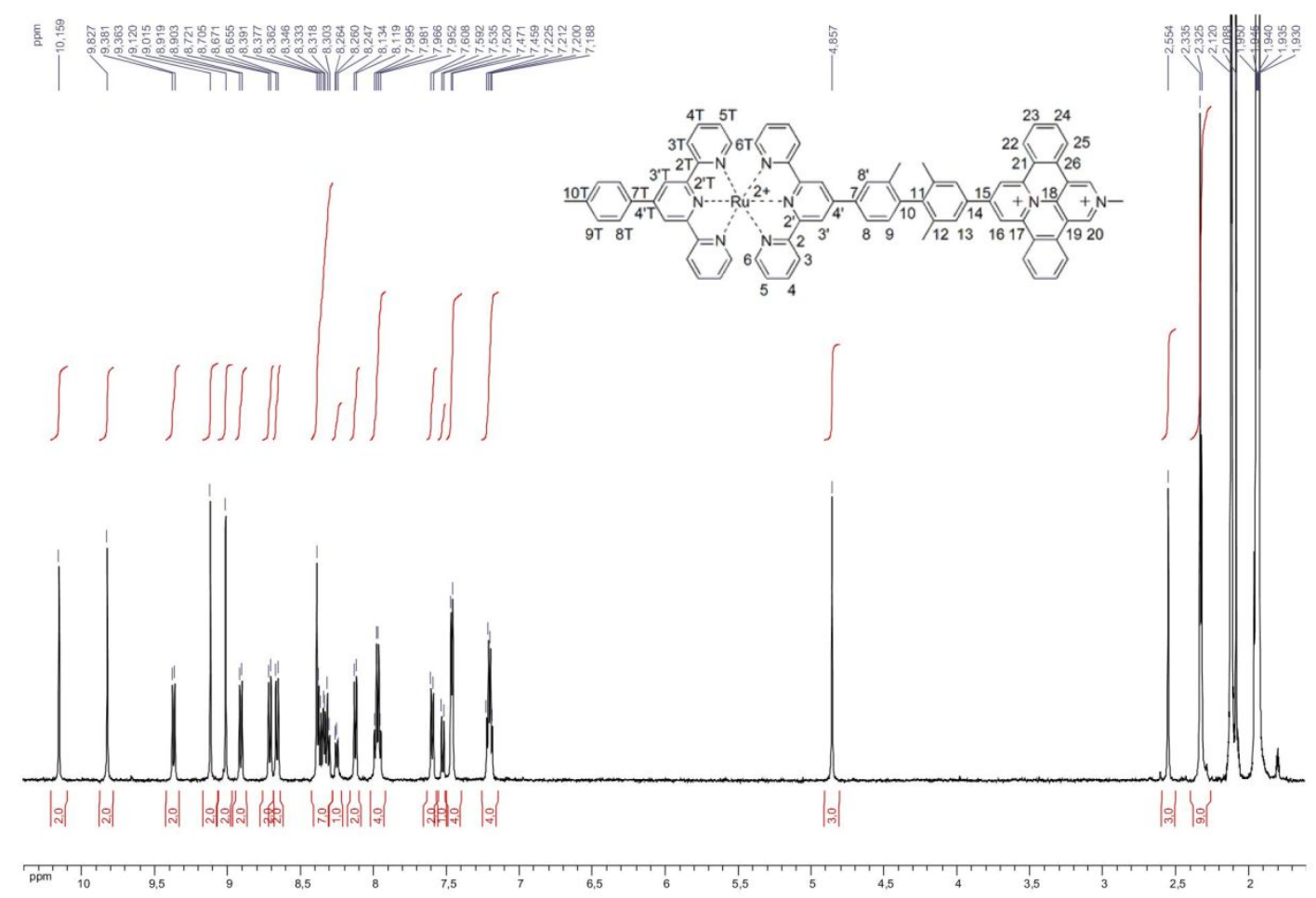

Figure S-13a. ${ }^{1} \mathrm{H}$ NMR spectrum $(500 \mathrm{MHz})$ of 3 in $\mathrm{CD}_{3} \mathrm{CN}$ (full spectrum).

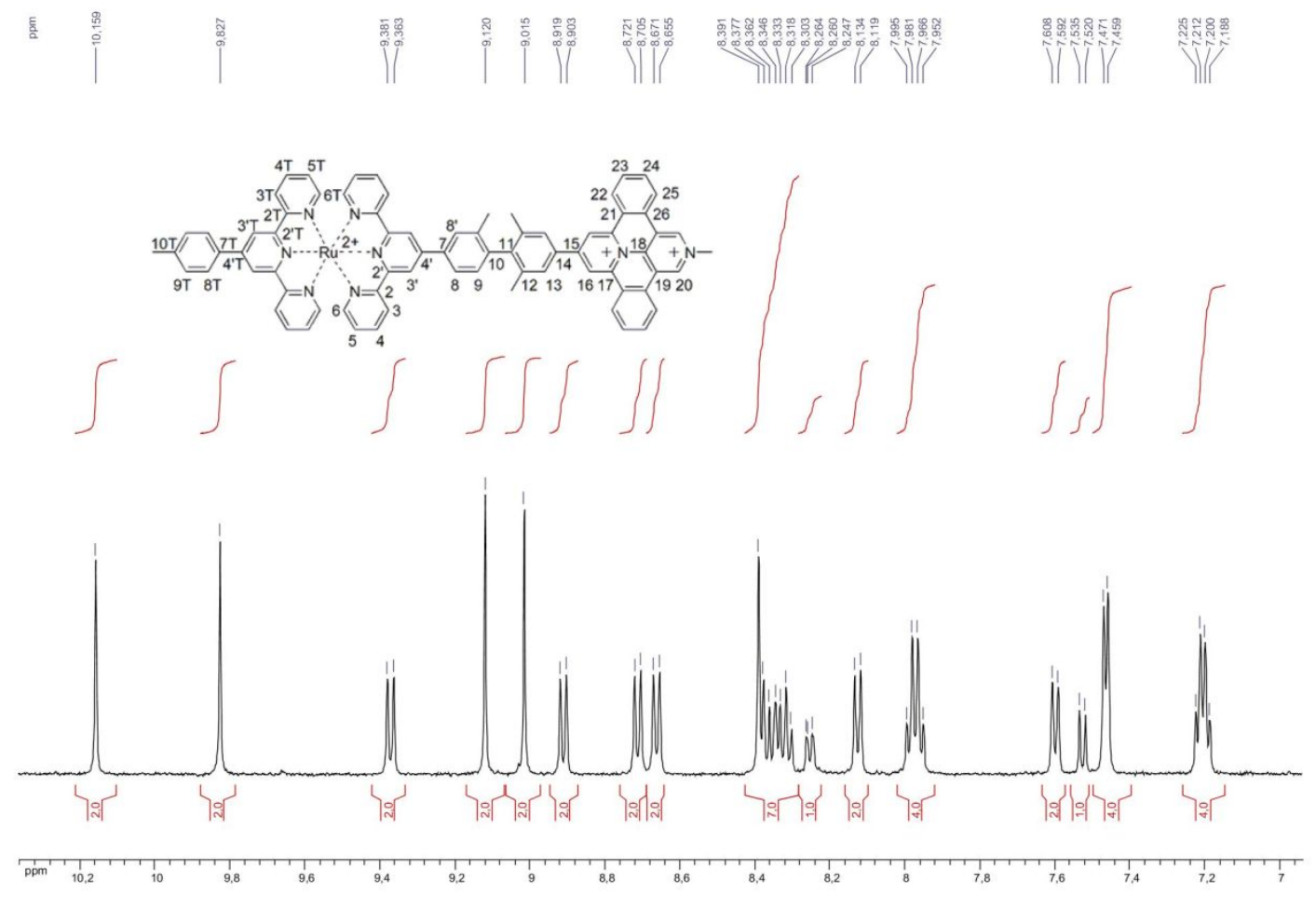

Figure $\mathbf{S}-13 b .{ }^{1} \mathrm{H}$ NMR spectrum $(500 \mathrm{MHz})$ of $\mathbf{3}$ in $\mathrm{CD}_{3} \mathrm{CN}$ (aromatic part). 


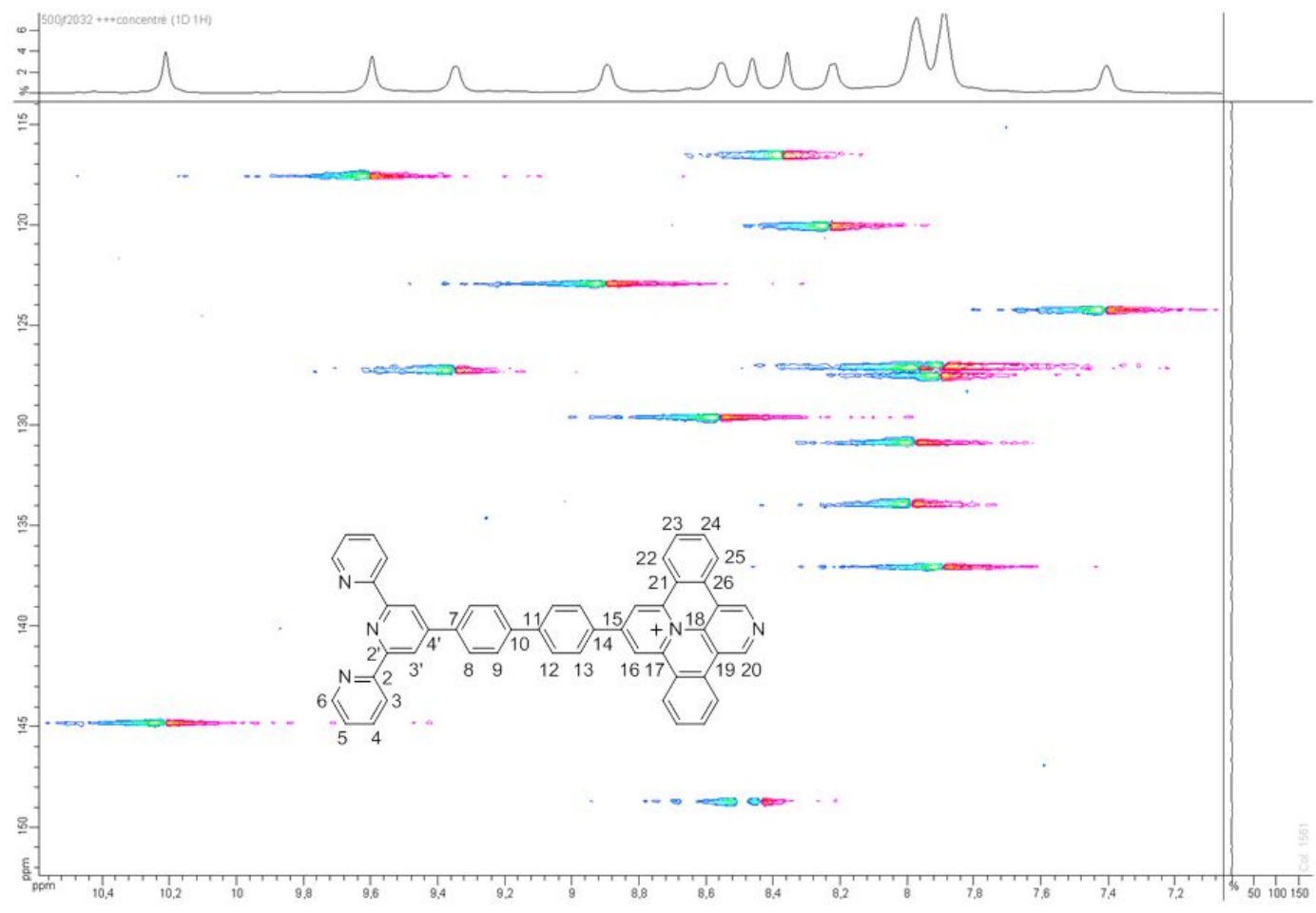

Figure S-14a. HSQC NMR spectrum (500 and $126 \mathrm{MHz}$ ) of ligand 1 in DMSO- $d^{6}$ (aromatic part).

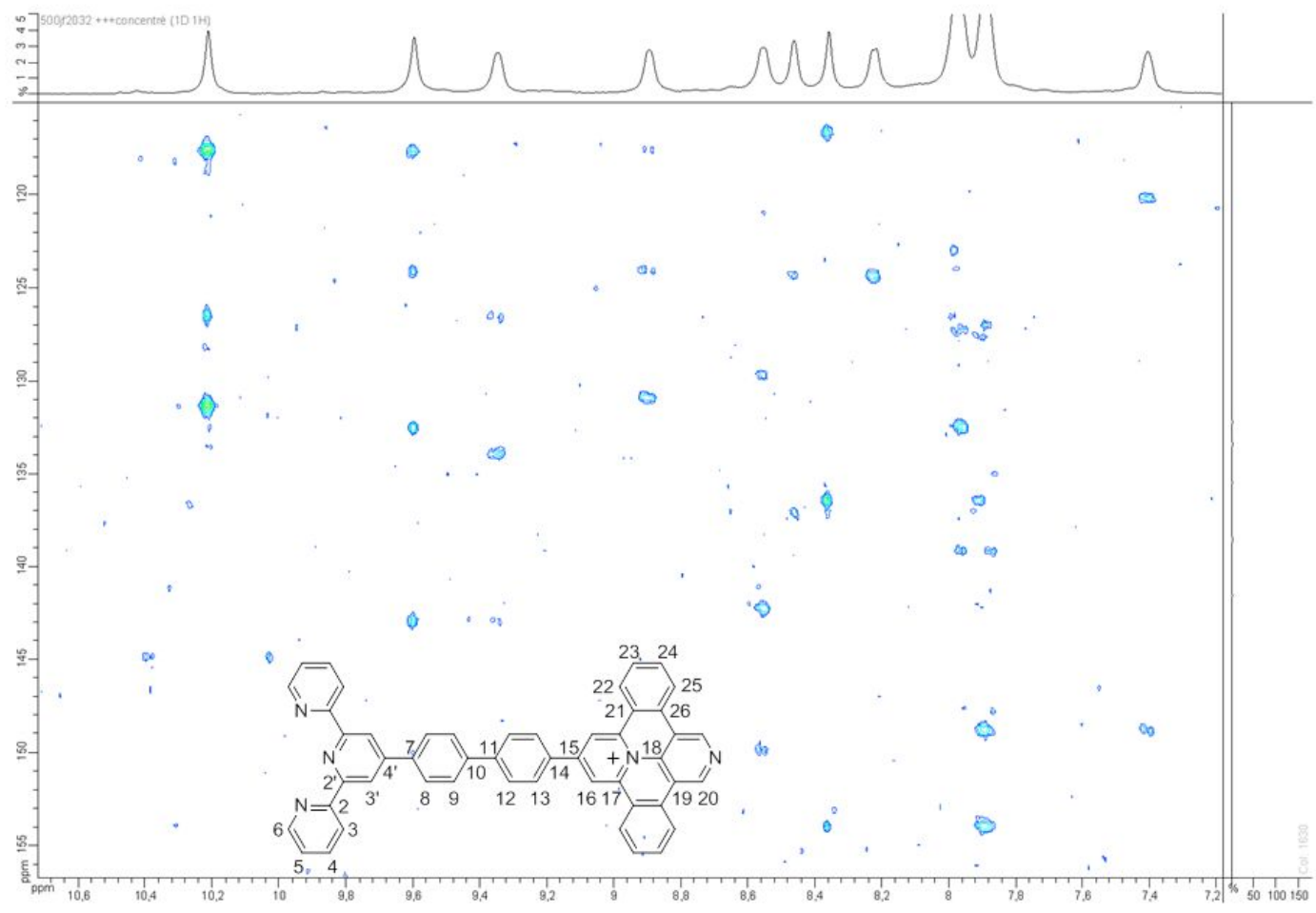

Figure S-14b. HMBC NMR spectrum (500 and $126 \mathrm{MHz}$ ) of ligand 1 in DMSO- $d^{6}$ (aromatic part). 


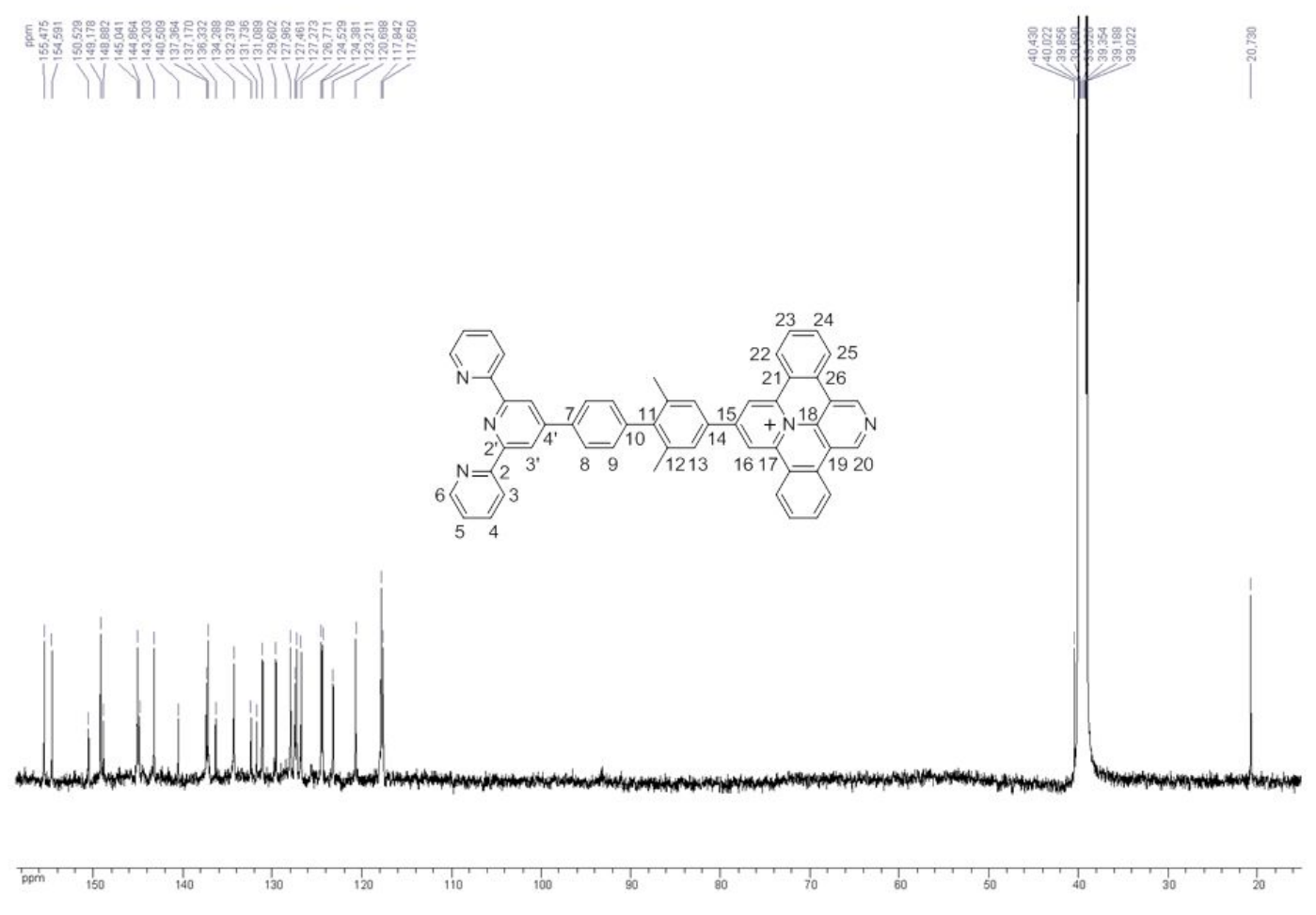

Figure S-15a. ${ }^{13} \mathrm{C}$ NMR spectrum (126 MHz) of ligand 2 in DMSO- $d^{6}$ (full spectrum).
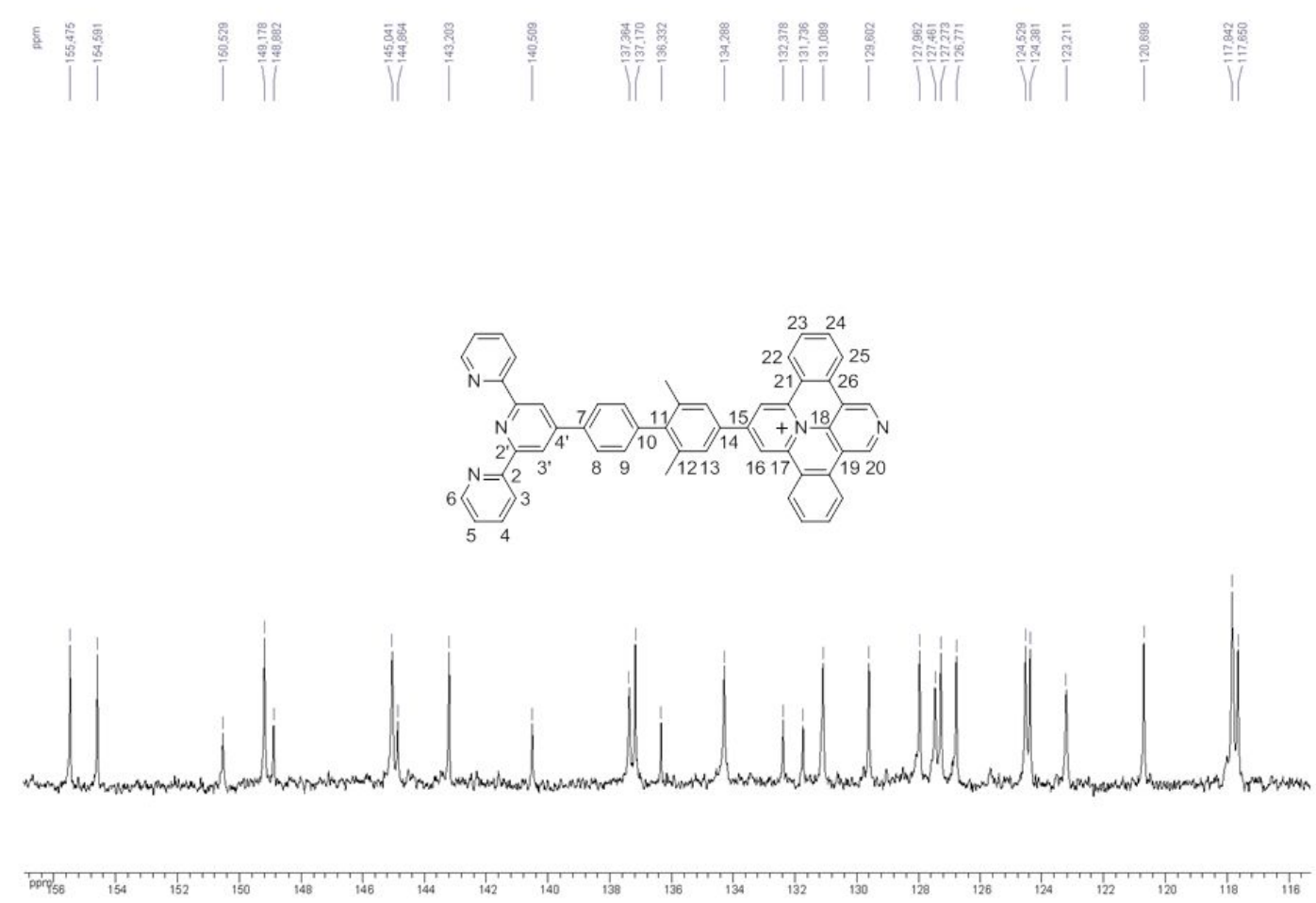

Figure S-15b. ${ }^{13} \mathrm{C}$ NMR spectrum $(126 \mathrm{MHz})$ of ligand 2 in DMSO- $d^{6}$ (aromatic part). 


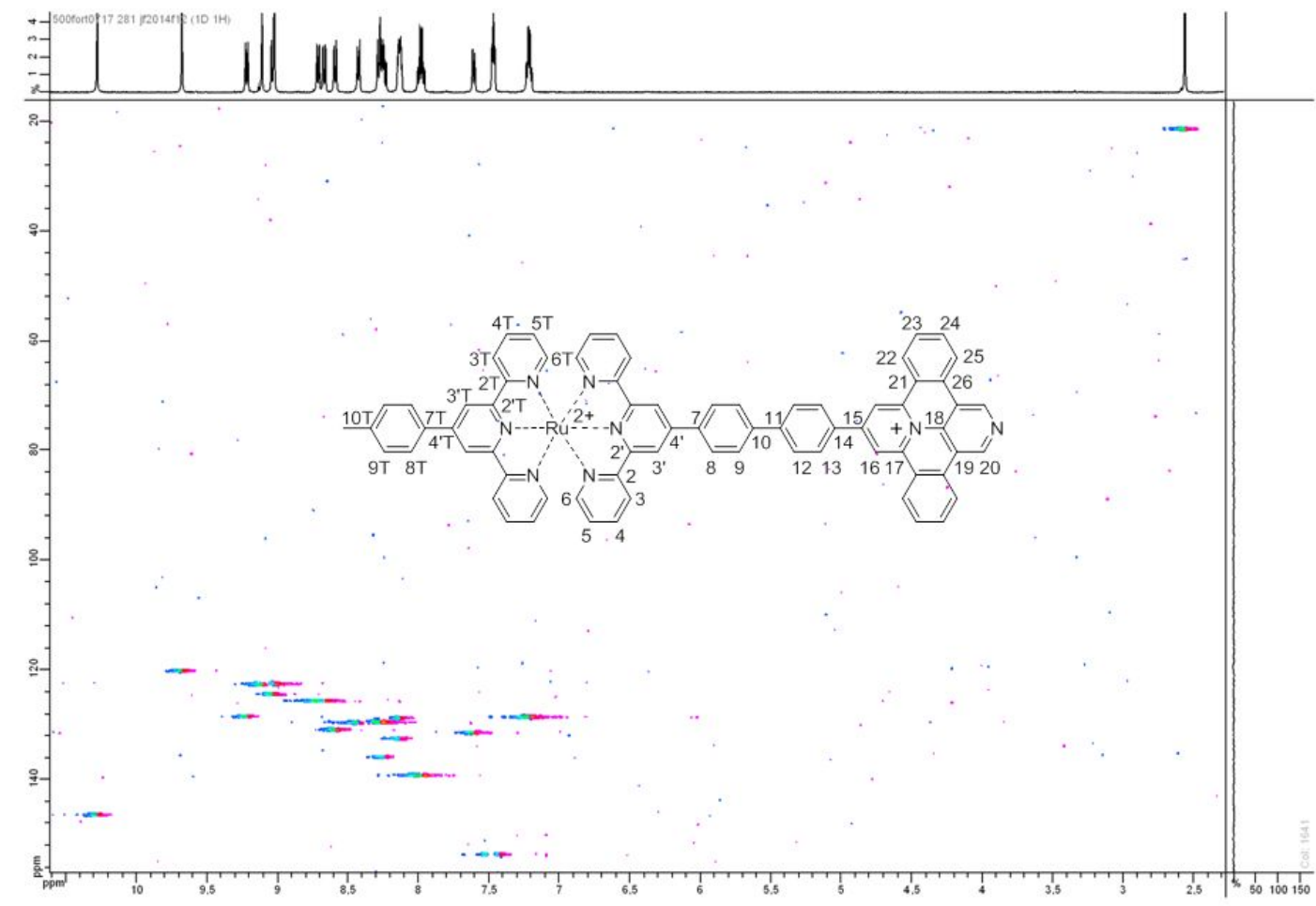

Figure S-16a. HSQC NMR spectrum (500 and $126 \mathrm{MHz}$ ) of dyad 1 in $\mathrm{CD}_{3} \mathrm{CN}$ (full spectrum).

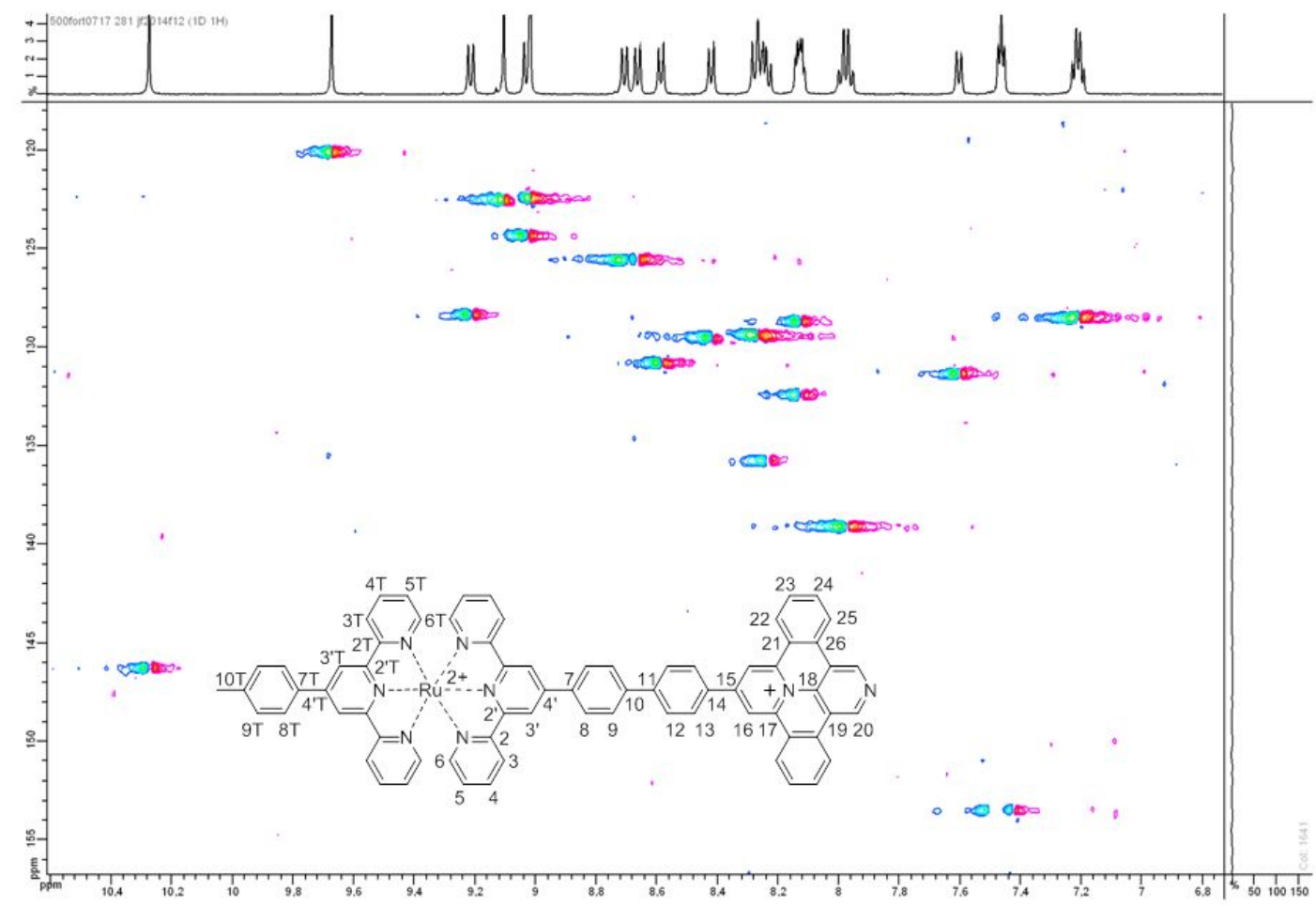

Figure S-16b. HSQC NMR spectrum (500 and $126 \mathrm{MHz}$ ) of dyad 1 in $\mathrm{CD}_{3} \mathrm{CN}$ (aromatic part). 


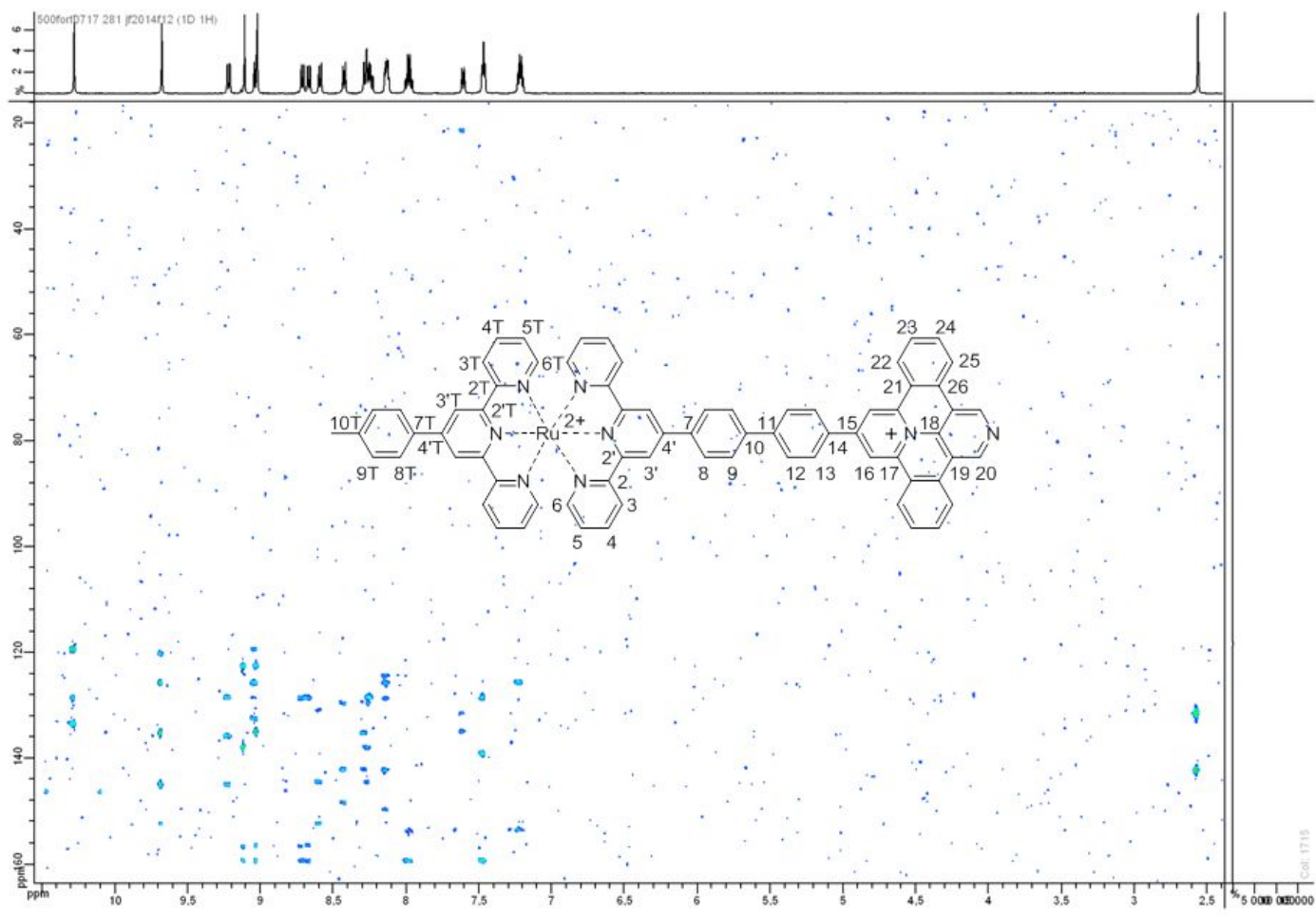

Figure S-16c. HMBC NMR spectrum (500 and $126 \mathrm{MHz}$ ) of dyad 1 in $\mathrm{CD}_{3} \mathrm{CN}$ (full spectrum).

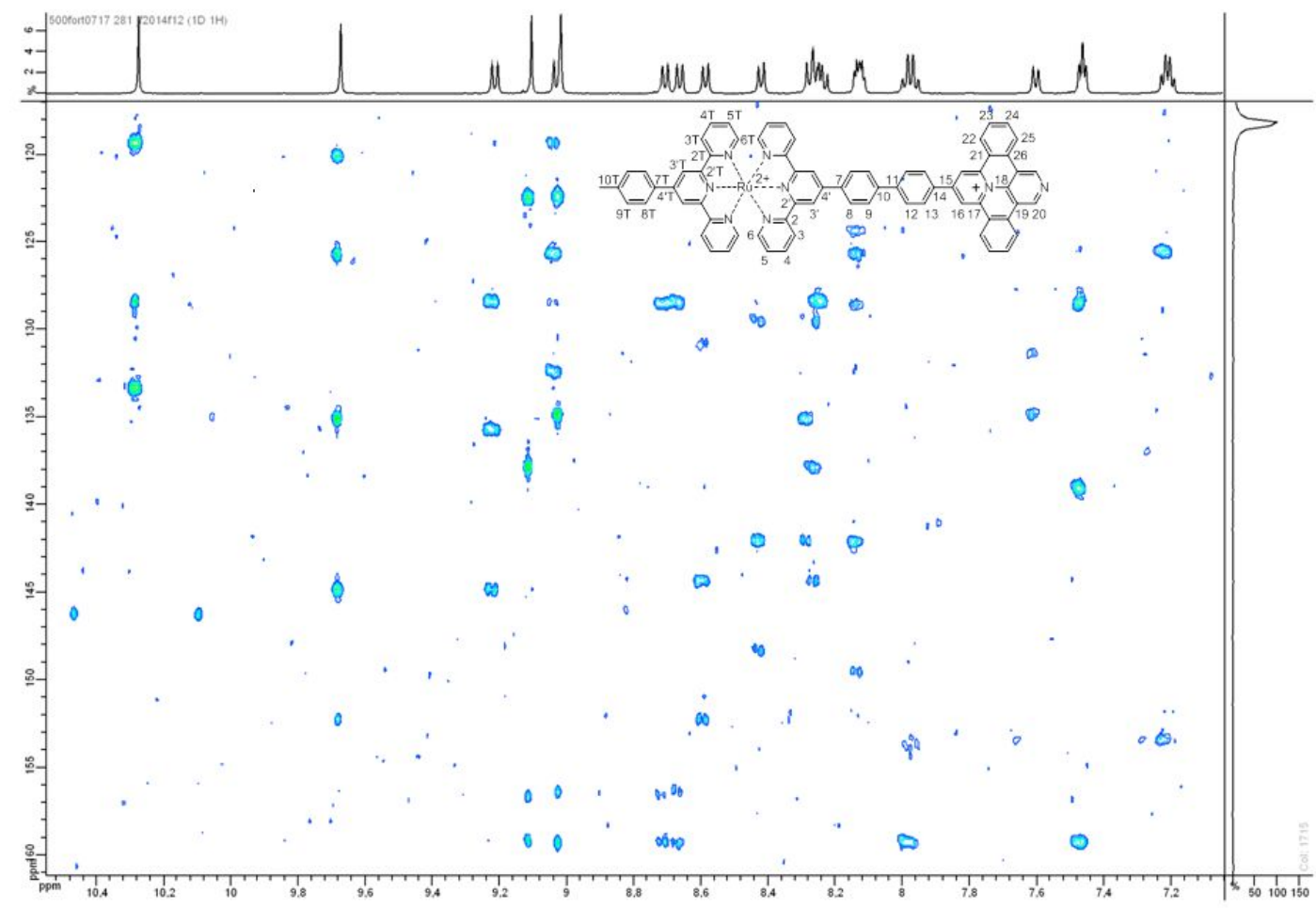

Figure S-16d. HMBC NMR spectrum (500 and $126 \mathrm{MHz}$ ) of dyad 1 in $\mathrm{CD}_{3} \mathrm{CN}$ (aromatic part). 


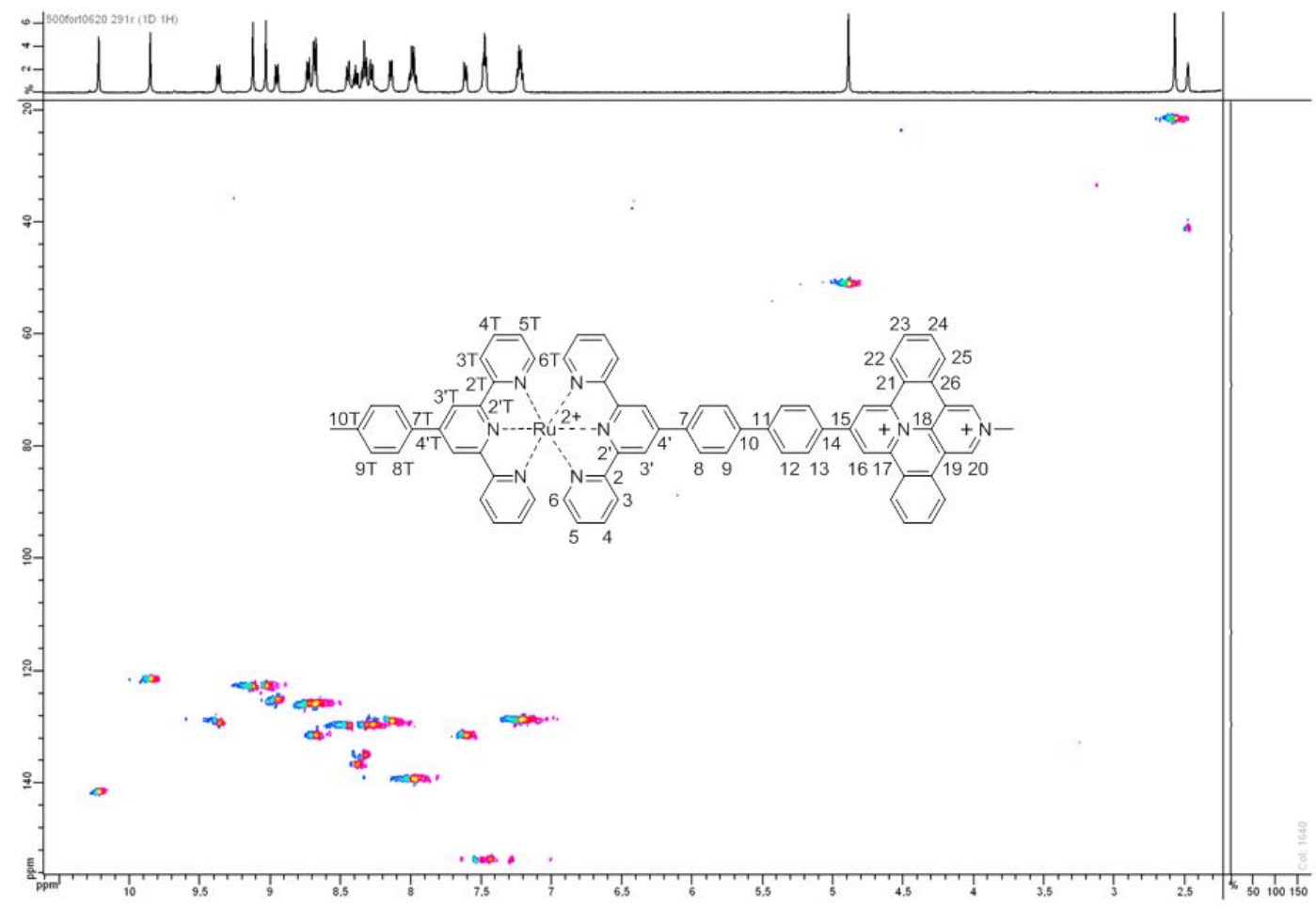

Figure S-17a. HSQC NMR spectrum (500 and $126 \mathrm{MHz}$ ) of 1 in $\mathrm{CD}_{3} \mathrm{CN}$ (full spectrum).

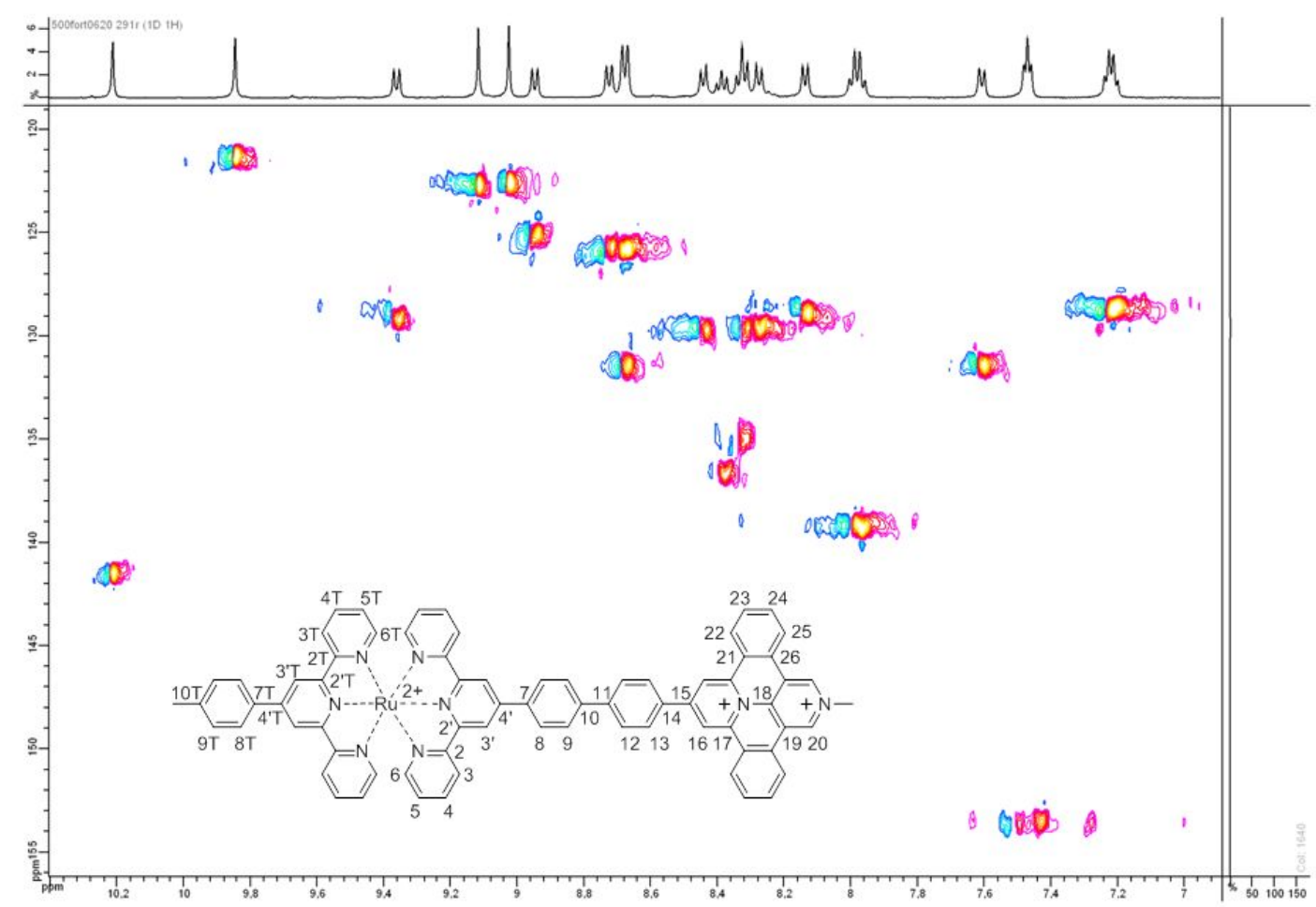

Figure S-17b. HSQC NMR spectrum (500 and $126 \mathrm{MHz}$ ) of $\mathbf{1}$ in $\mathrm{CD}_{3} \mathrm{CN}$ (aromatic part). 


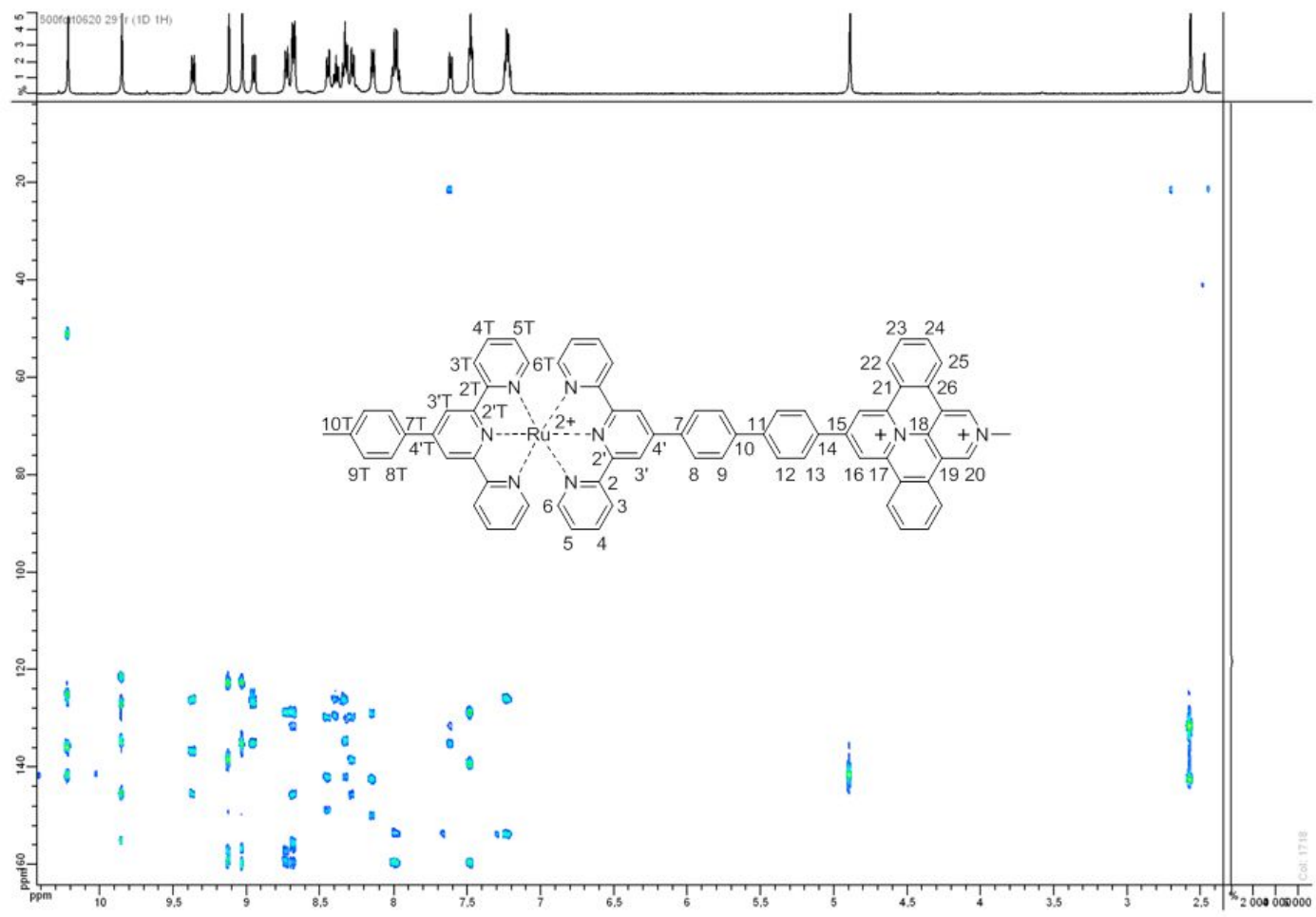

Figure S-17c. HMBC NMR spectrum (500 and $126 \mathrm{MHz}$ ) of 1 in $\mathrm{CD}_{3} \mathrm{CN}$ (full spectrum).

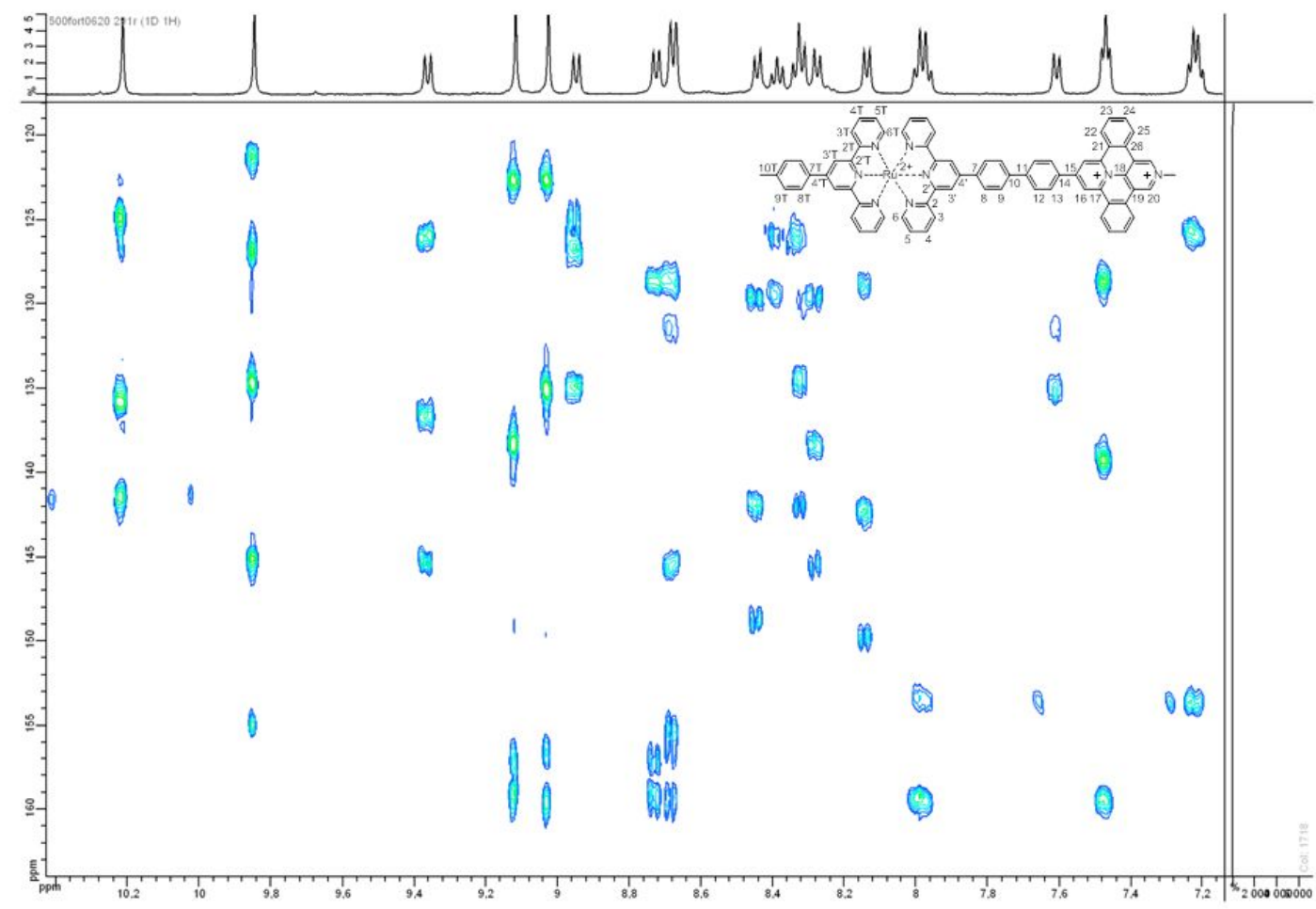

Figure S-17d. HMBC NMR spectrum (500 and $126 \mathrm{MHz}$ ) of 1 in $\mathrm{CD}_{3} \mathrm{CN}$ (aromatic part). 


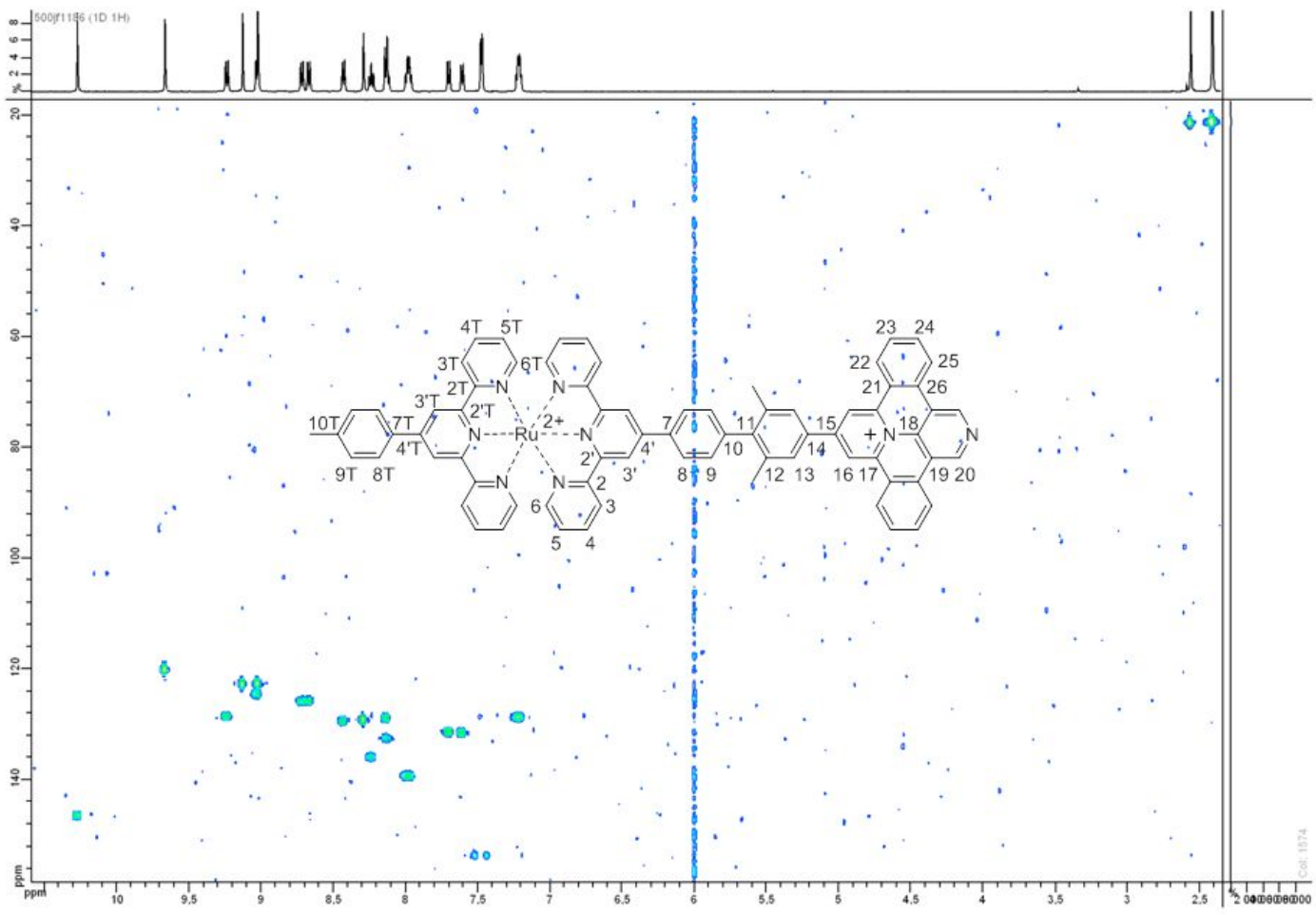

Figure S-18a. HSQC NMR spectrum (500 and $126 \mathrm{MHz}$ ) of dyad 2 in $\mathrm{CD}_{3} \mathrm{CN}$ (full spectrum).

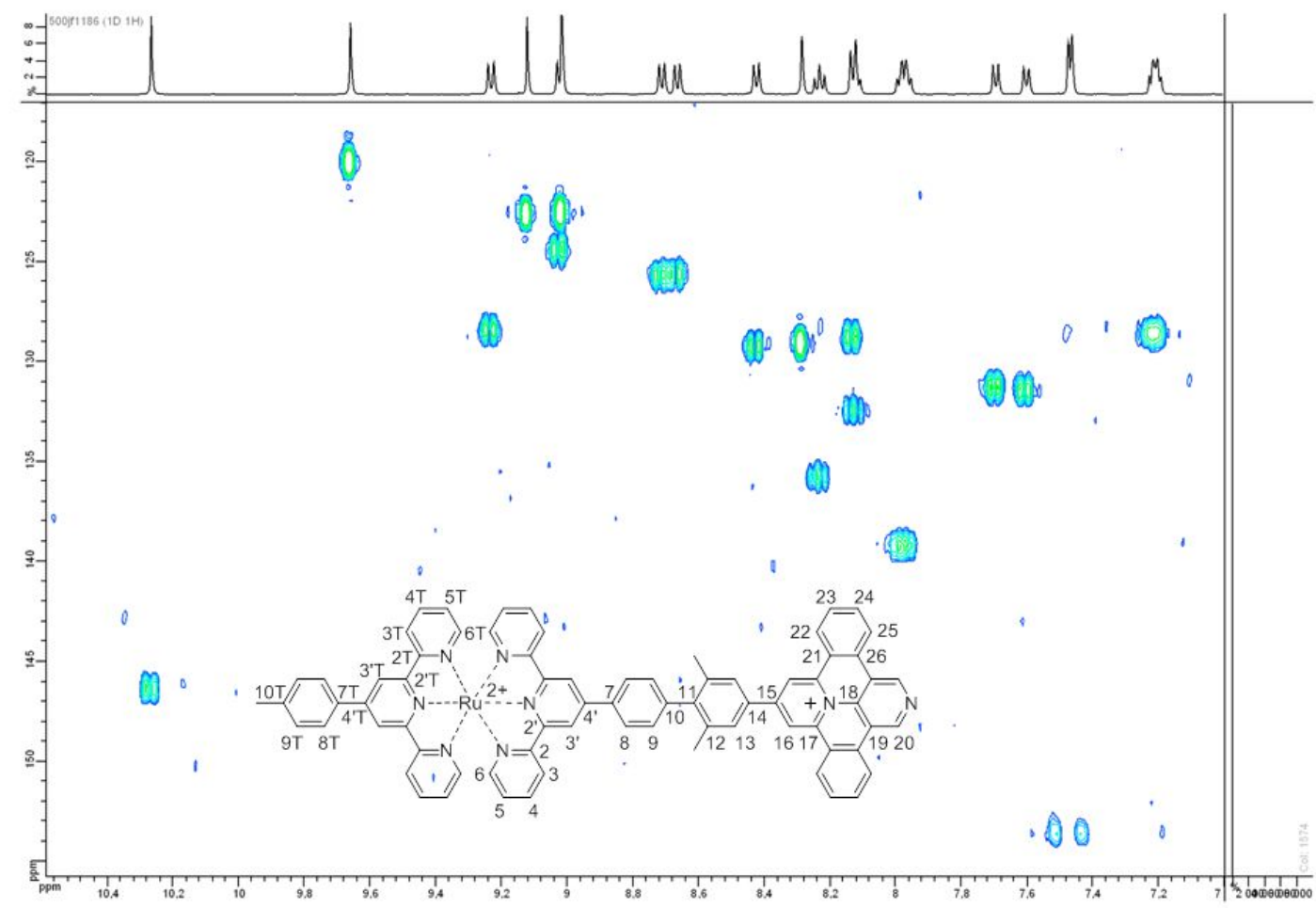

Figure S-18b. HSQC NMR spectrum (500 and $126 \mathrm{MHz}$ ) of dyad 2 in $\mathrm{CD}_{3} \mathrm{CN}$ (aromatic part). 


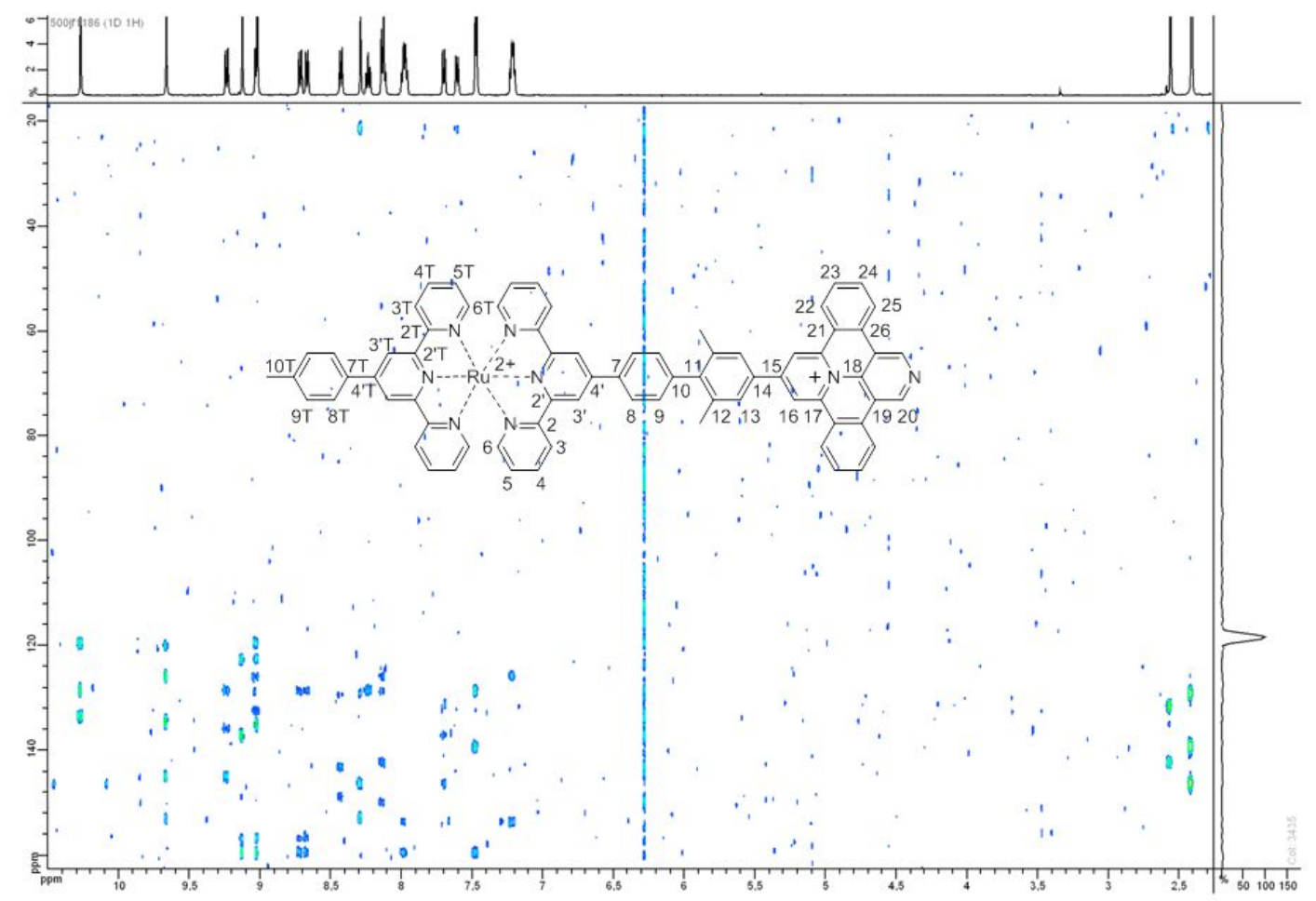

Figure S-18c. HMBC NMR spectrum (500 and $126 \mathrm{MHz}$ ) of dyad 2 in $\mathrm{CD}_{3} \mathrm{CN}$ (full spectrum).

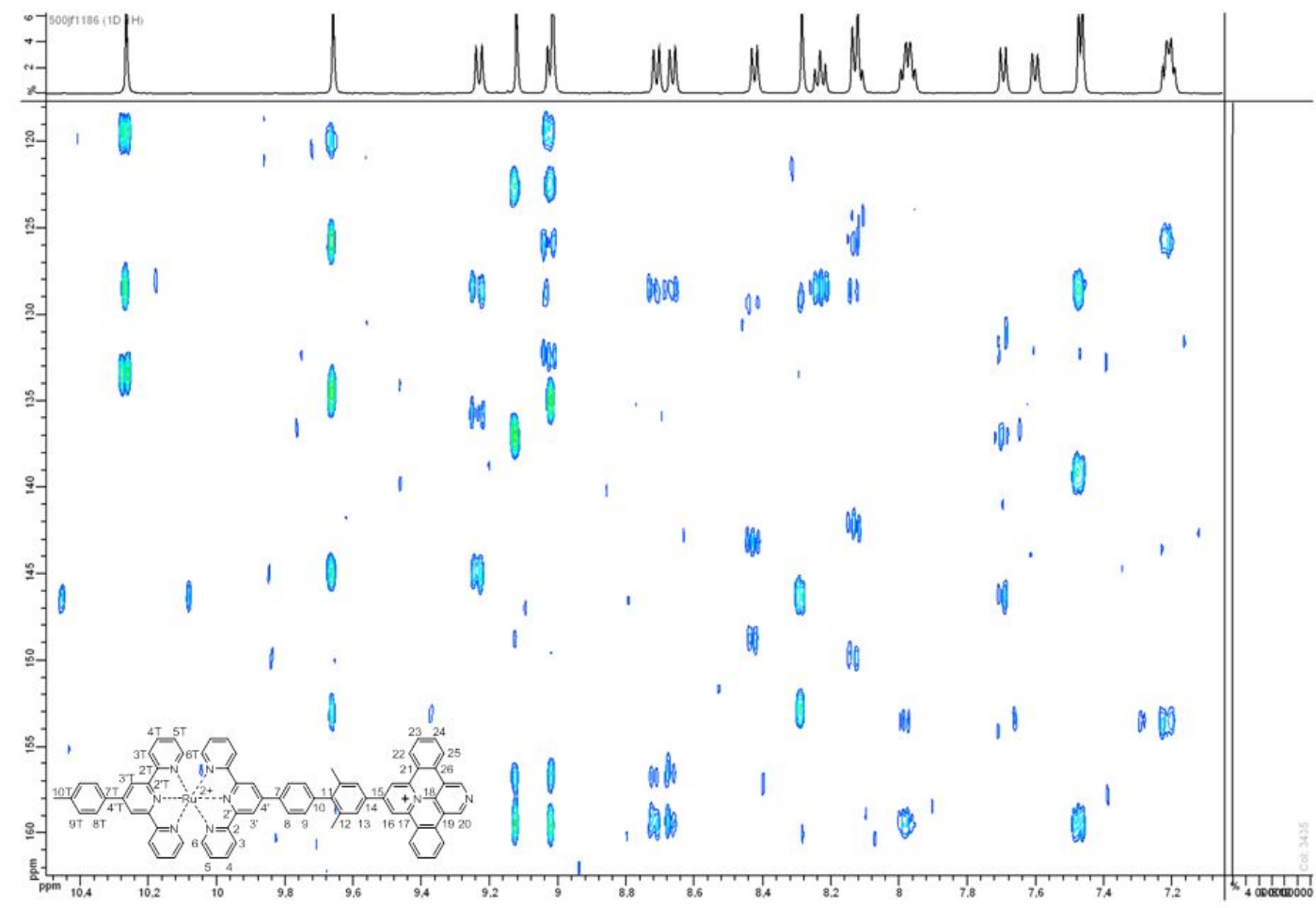

Figure S-18d. HMBC NMR spectrum (500 and $126 \mathrm{MHz}$ ) of dyad 2 in $\mathrm{CD}_{3} \mathrm{CN}$ (aromatic part). 


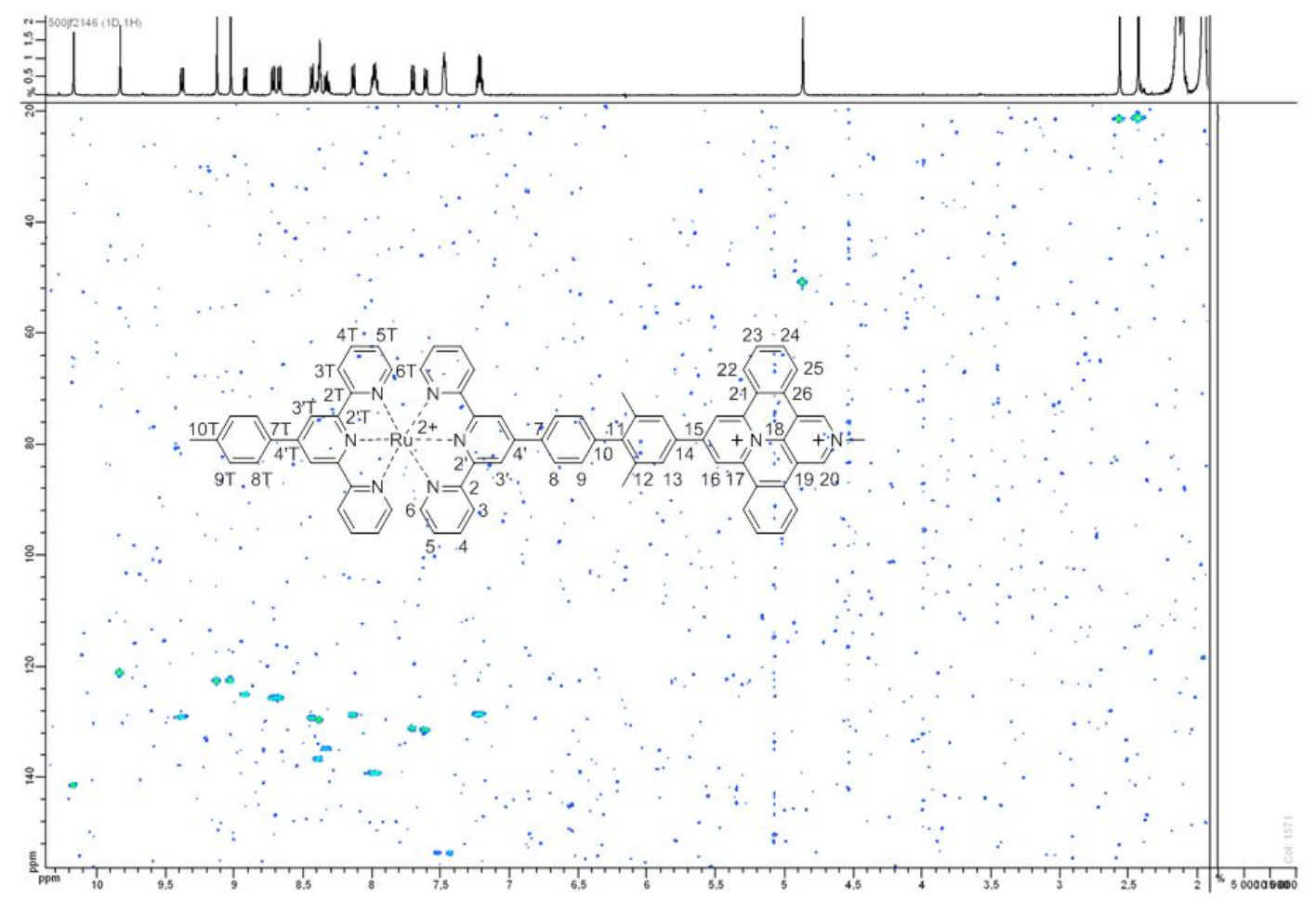

Figure S-19a. HSQC NMR spectrum (500 and $126 \mathrm{MHz}$ ) of 2 in $\mathrm{CD}_{3} \mathrm{CN}$ (full spectrum).

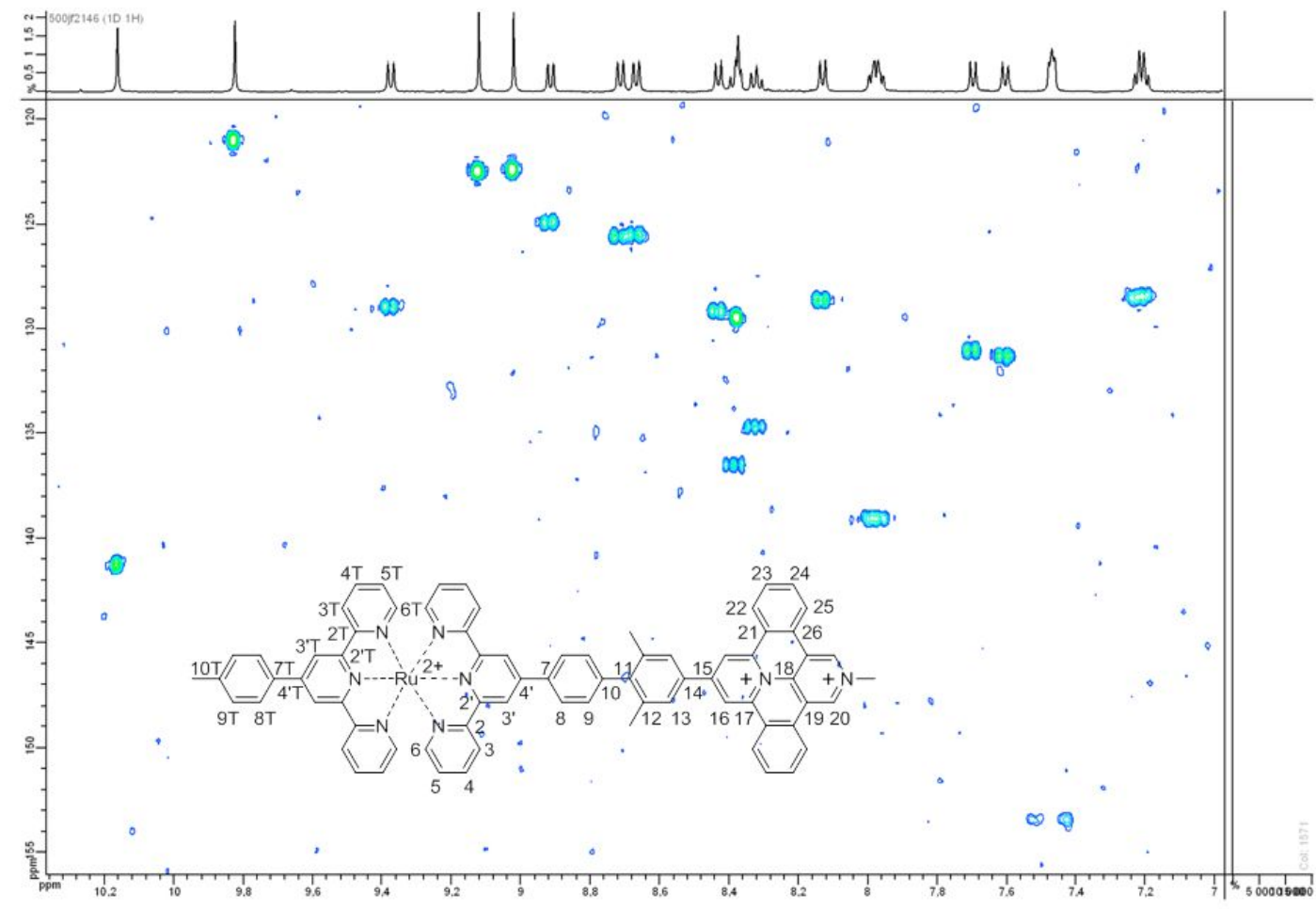

Figure S-19b. HSQC NMR spectrum (500 and $126 \mathrm{MHz}$ ) of 2 in $\mathrm{CD}_{3} \mathrm{CN}$ (aromatic part). 


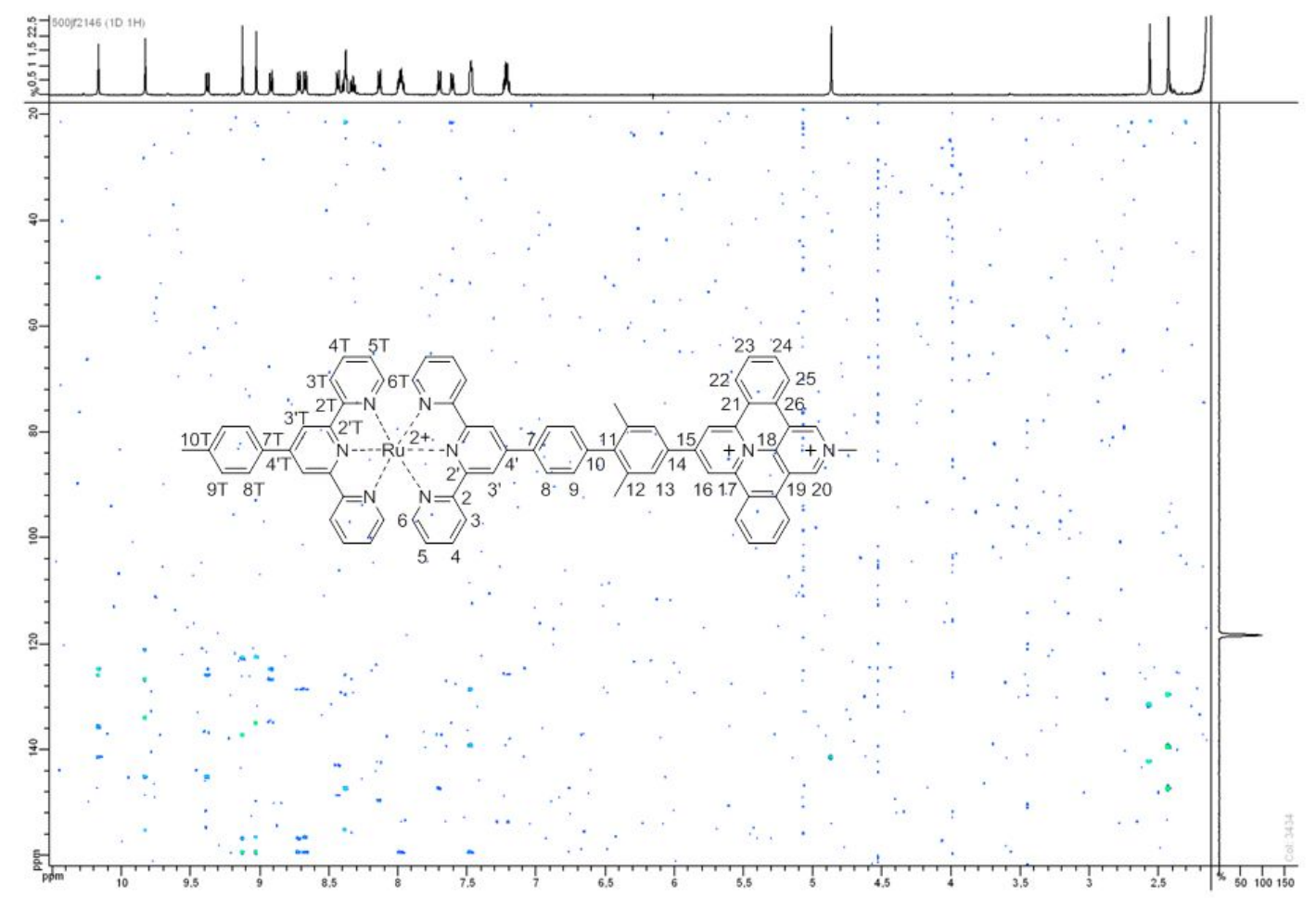

Figure S-19c. HMBC NMR spectrum (500 and $126 \mathrm{MHz}$ ) of 2 in $\mathrm{CD}_{3} \mathrm{CN}$ (full spectrum).

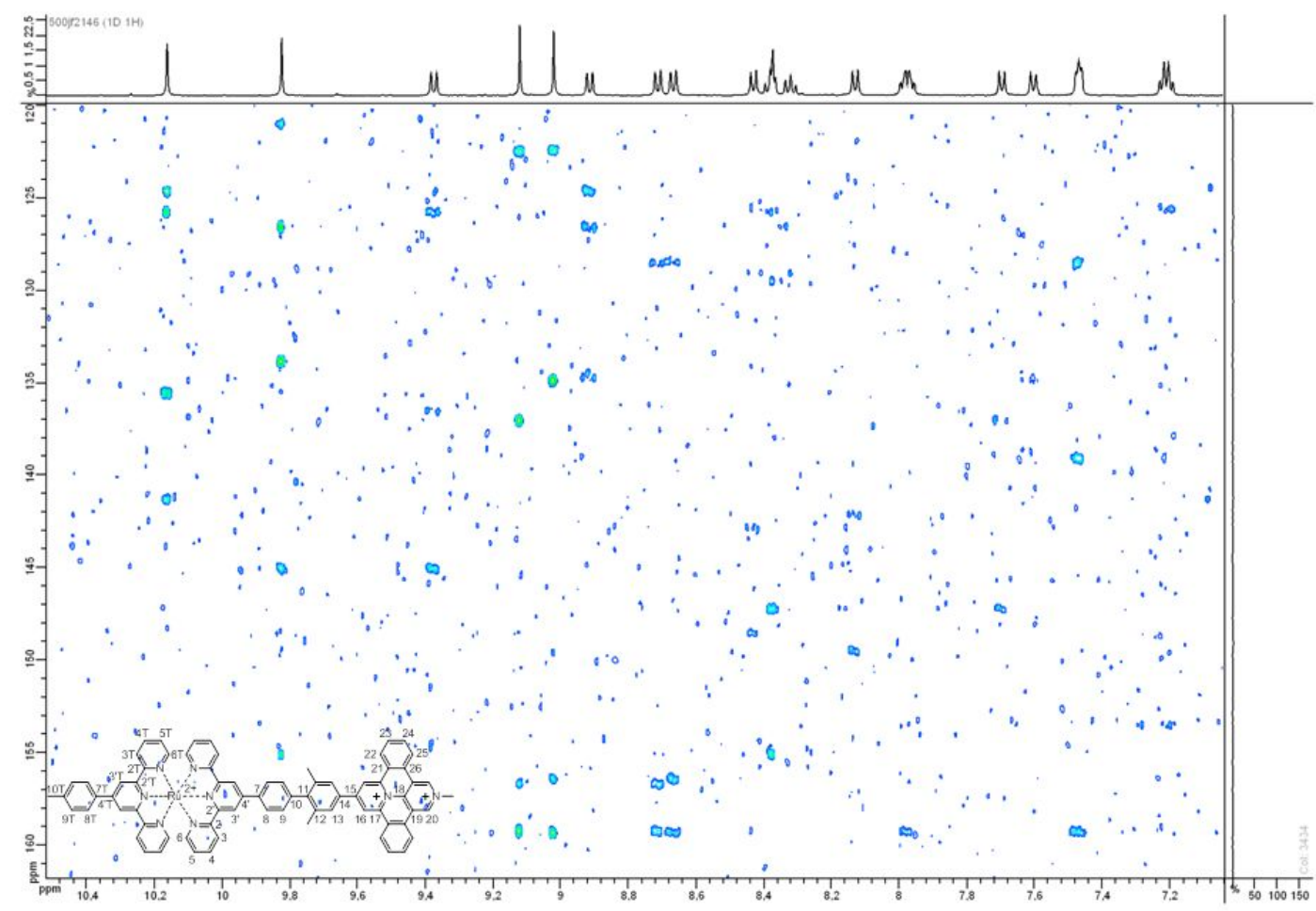

Figure S-19d. HMBC NMR spectrum (500 and $126 \mathrm{MHz}$ ) of 2 in $\mathrm{CD}_{3} \mathrm{CN}$ (aromatic part). 


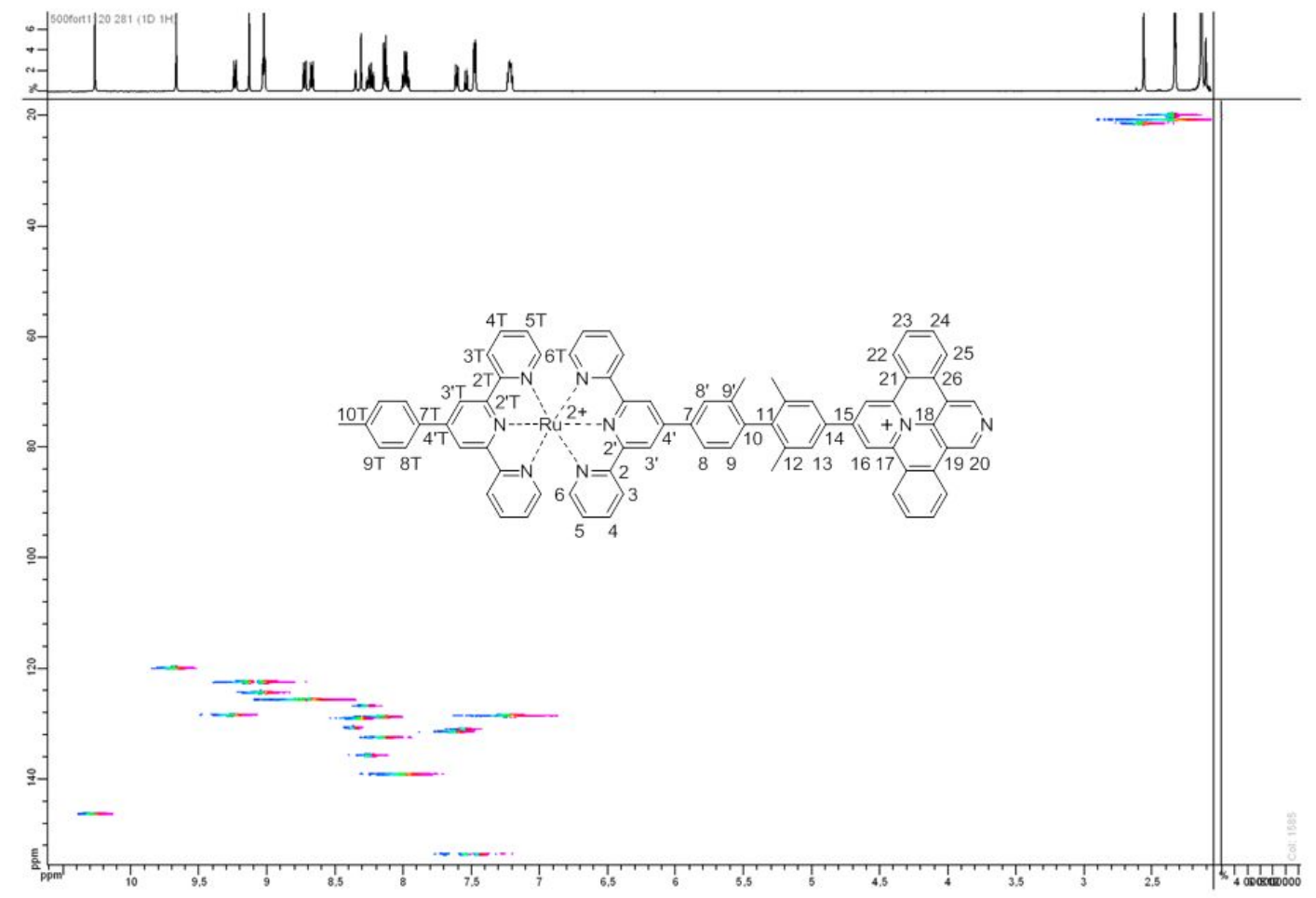

Figure S-20a. HSQC NMR spectrum (500 and $126 \mathrm{MHz}$ ) of dyad 3 in $\mathrm{CD}_{3} \mathrm{CN}$ (full spectrum).

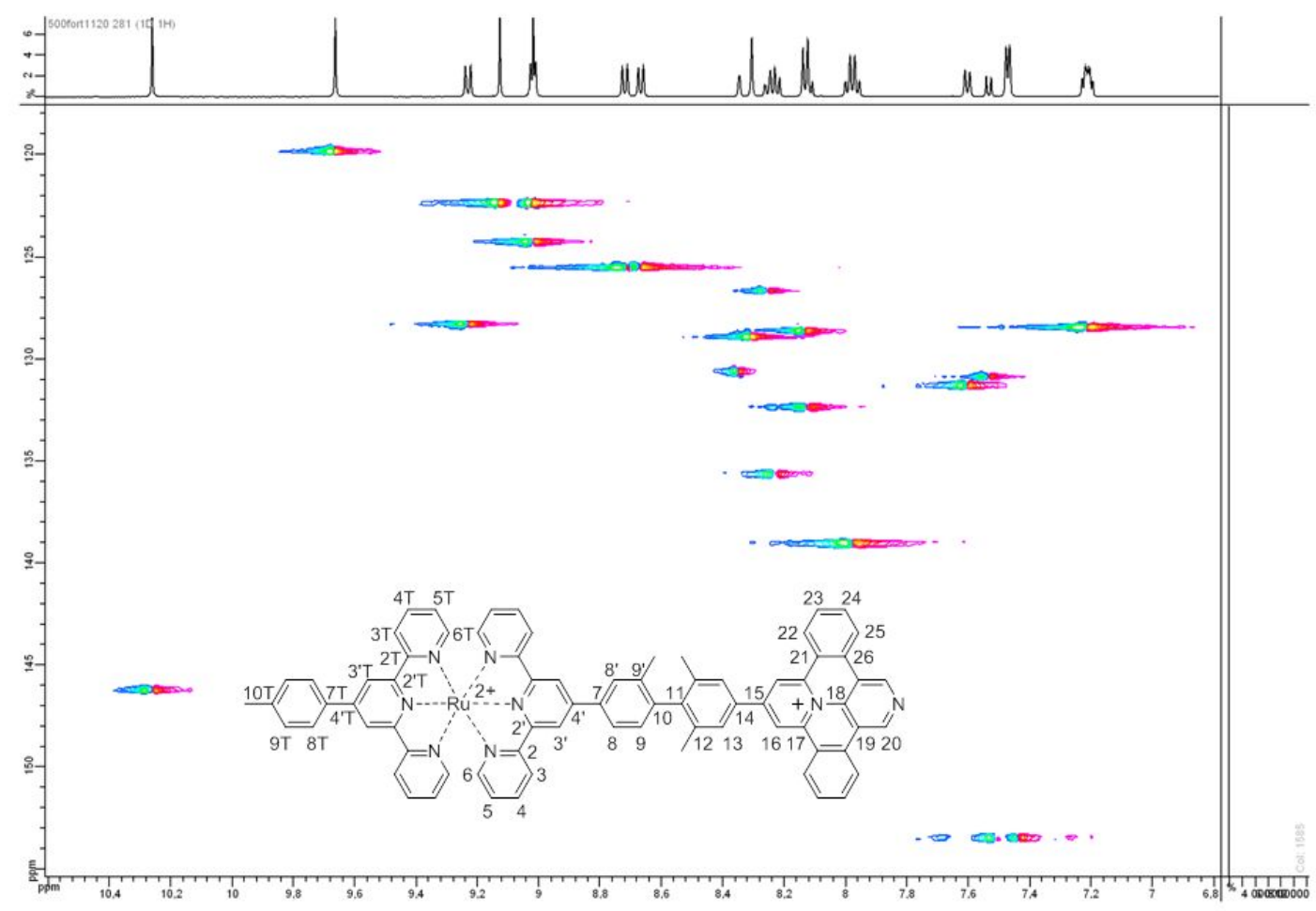

Figure S-20b. HSQC NMR spectrum (500 and $126 \mathrm{MHz}$ ) of dyad 3 in $\mathrm{CD}_{3} \mathrm{CN}$ (aromatic part). 


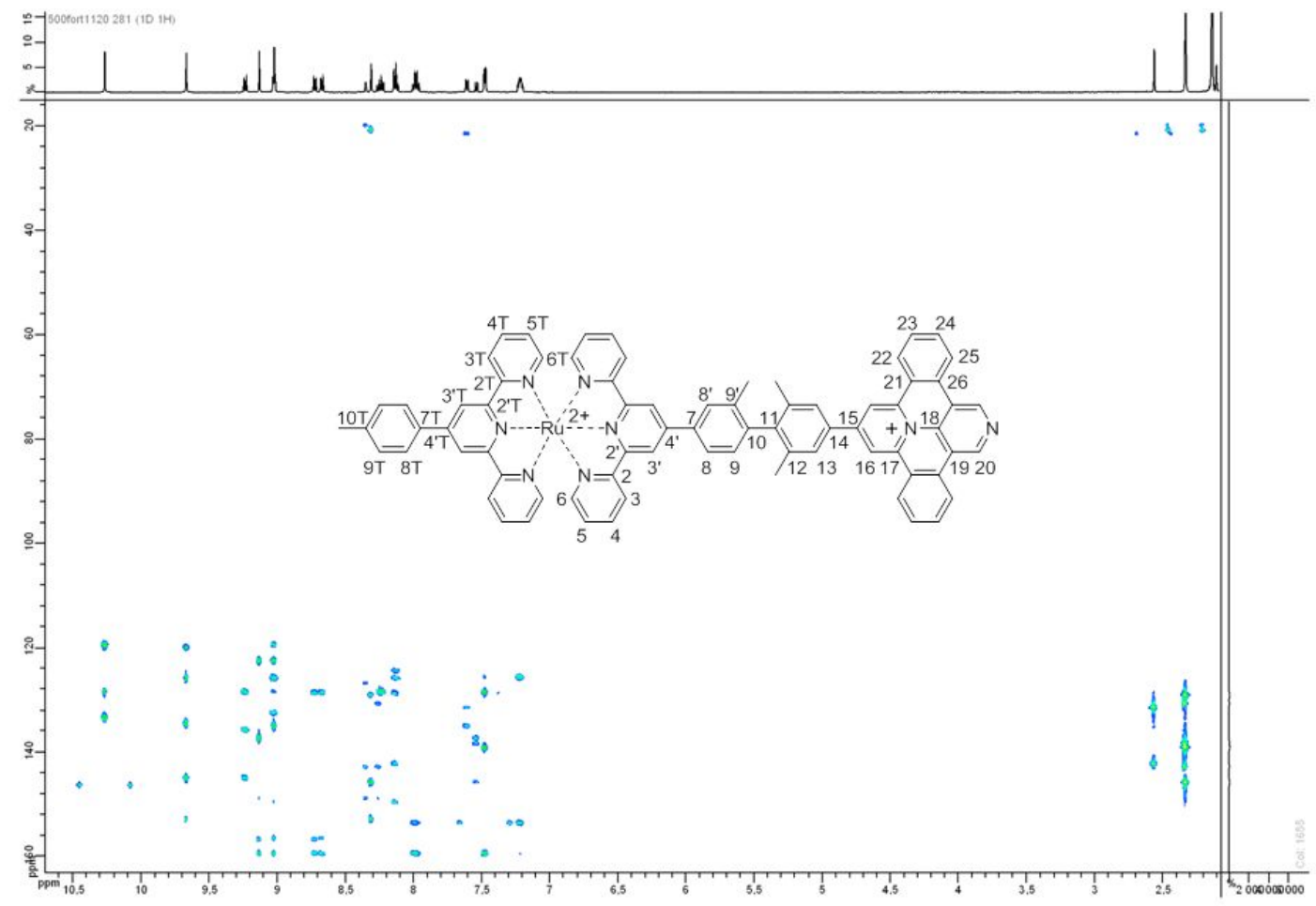

Figure S-20c. HMBC NMR spectrum (500 and $126 \mathrm{MHz}$ ) of dyad 3 in $\mathrm{CD}_{3} \mathrm{CN}$ (full spectrum).

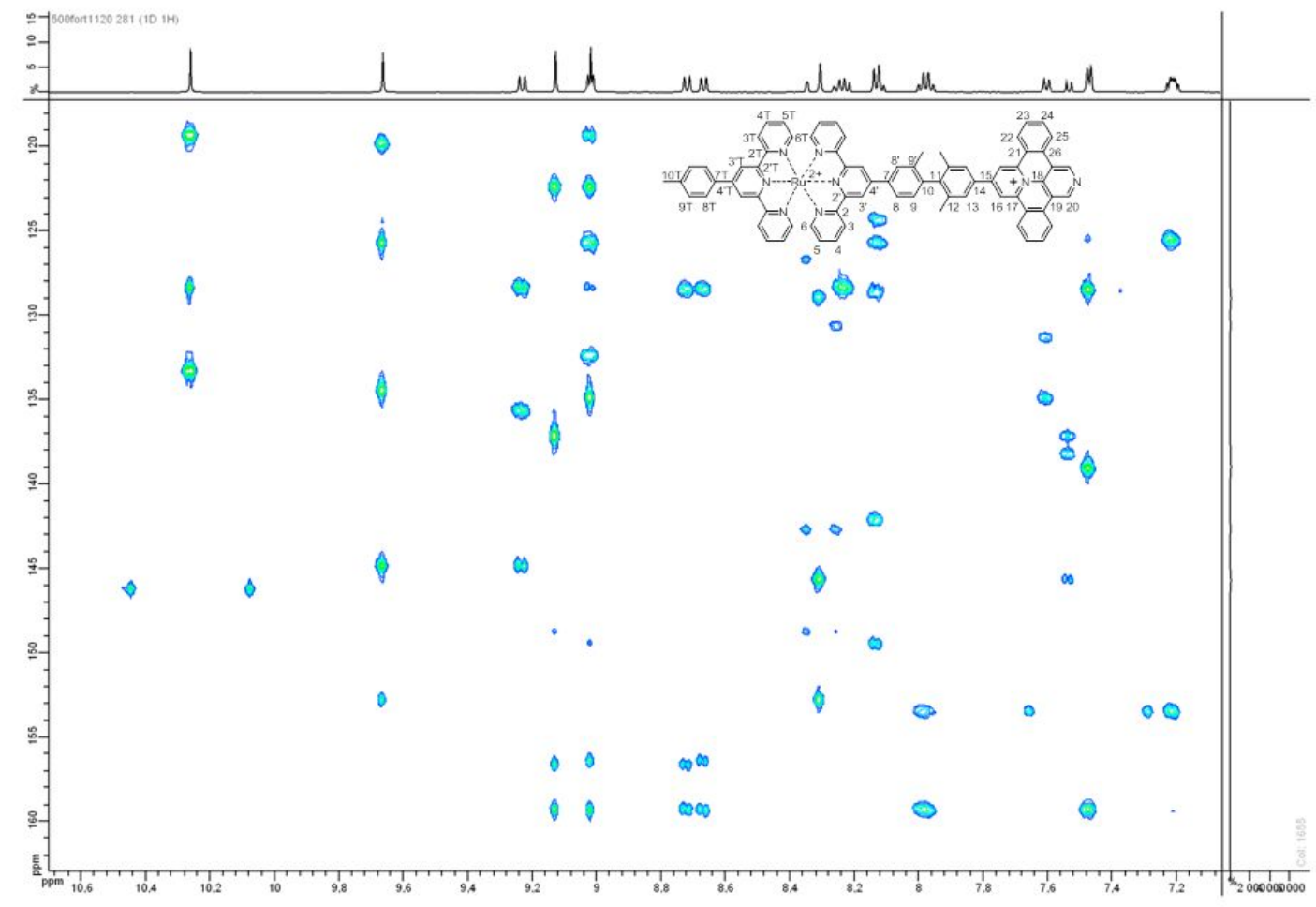

Figure S-20d. HMBC NMR spectrum (500 and $126 \mathrm{MHz}$ ) of dyad 3 in $\mathrm{CD}_{3} \mathrm{CN}$ (aromatic part). 


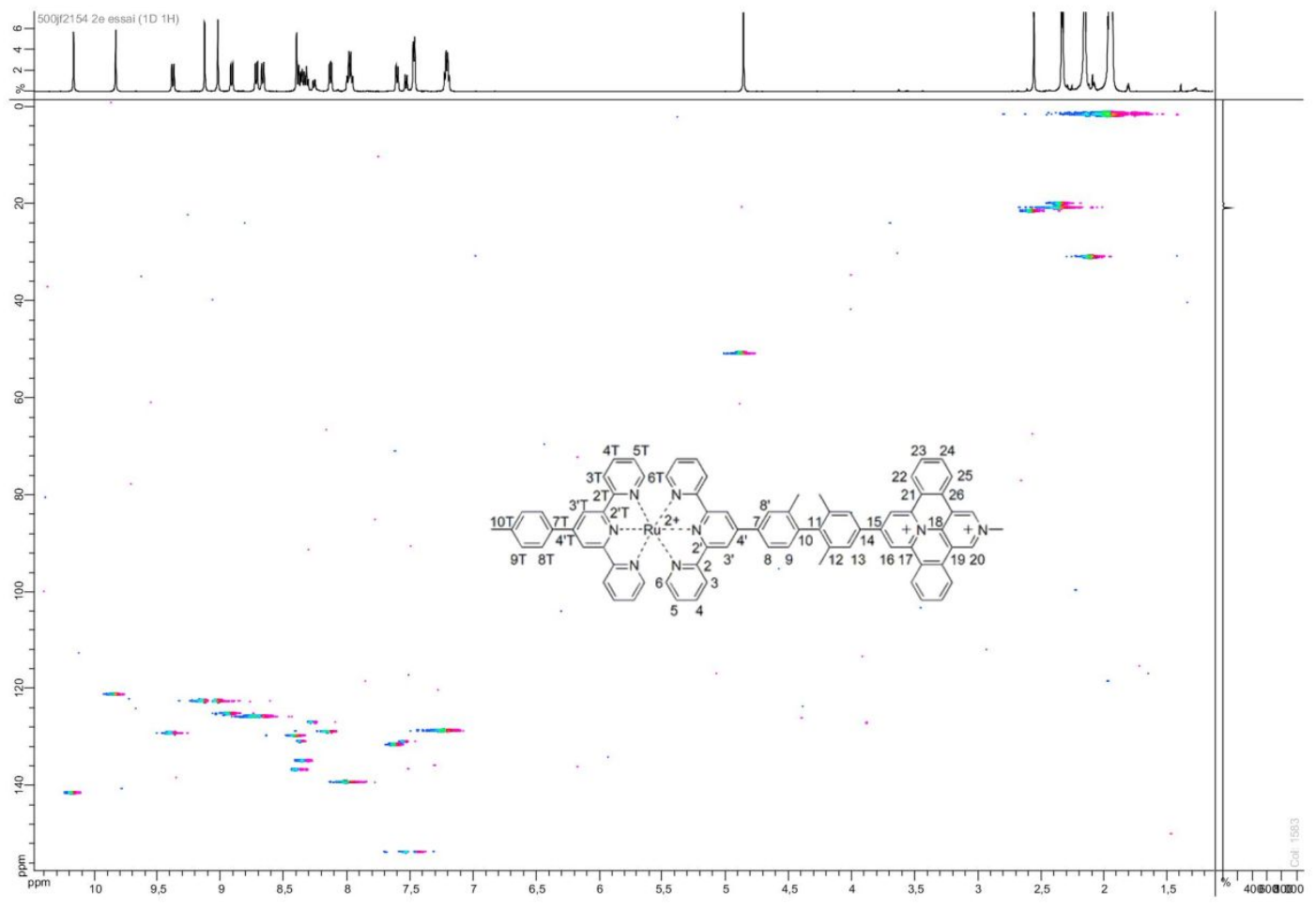

Figure S-21a. HSQC NMR spectrum (500 and $126 \mathrm{MHz}$ ) of 3 in $\mathrm{CD}_{3} \mathrm{CN}$ (full spectrum).

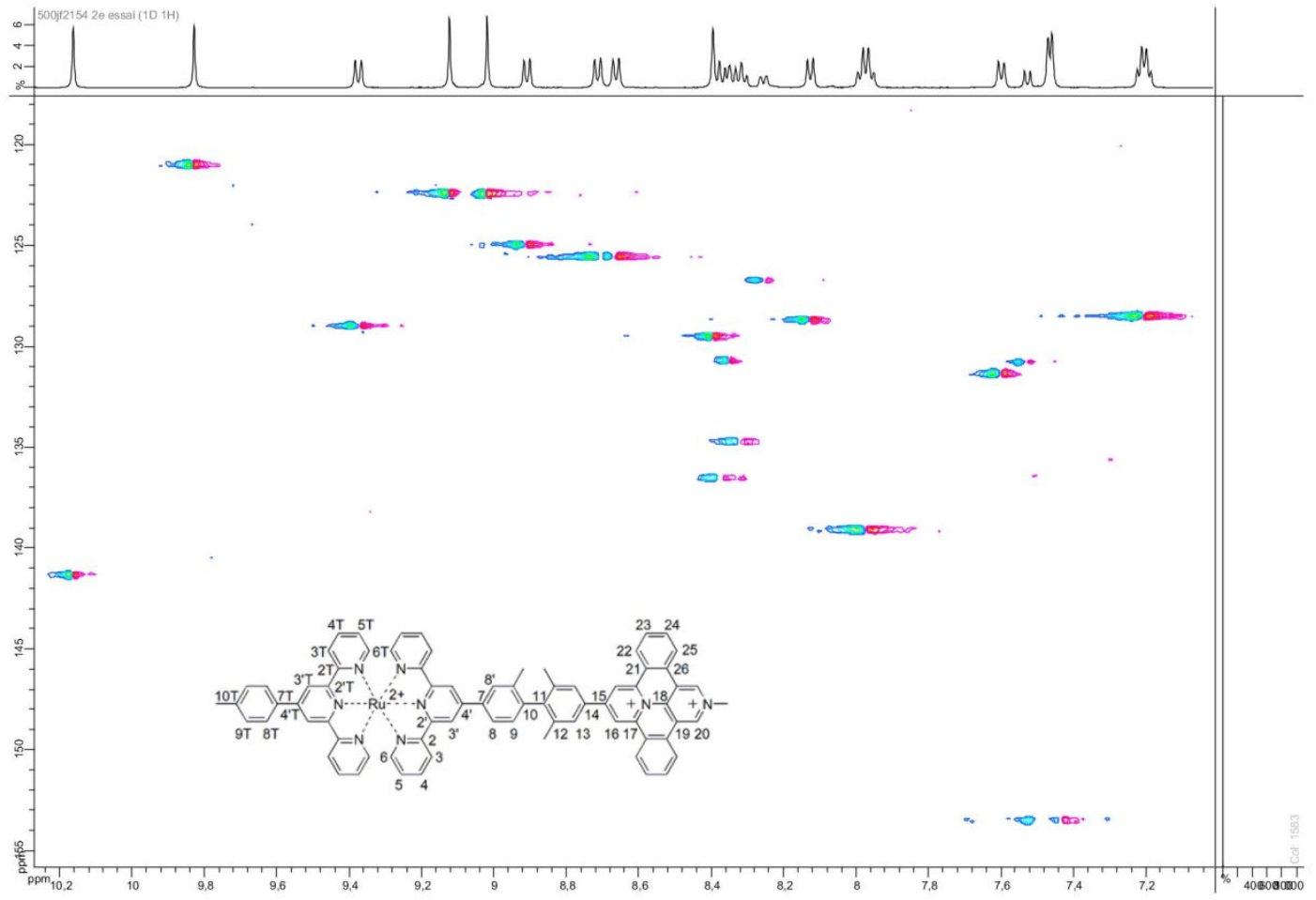

Figure S-21b. HSQC NMR spectrum (500 and $126 \mathrm{MHz}$ ) of $\mathbf{3}$ in $\mathrm{CD}_{3} \mathrm{CN}$ (aromatic part). 


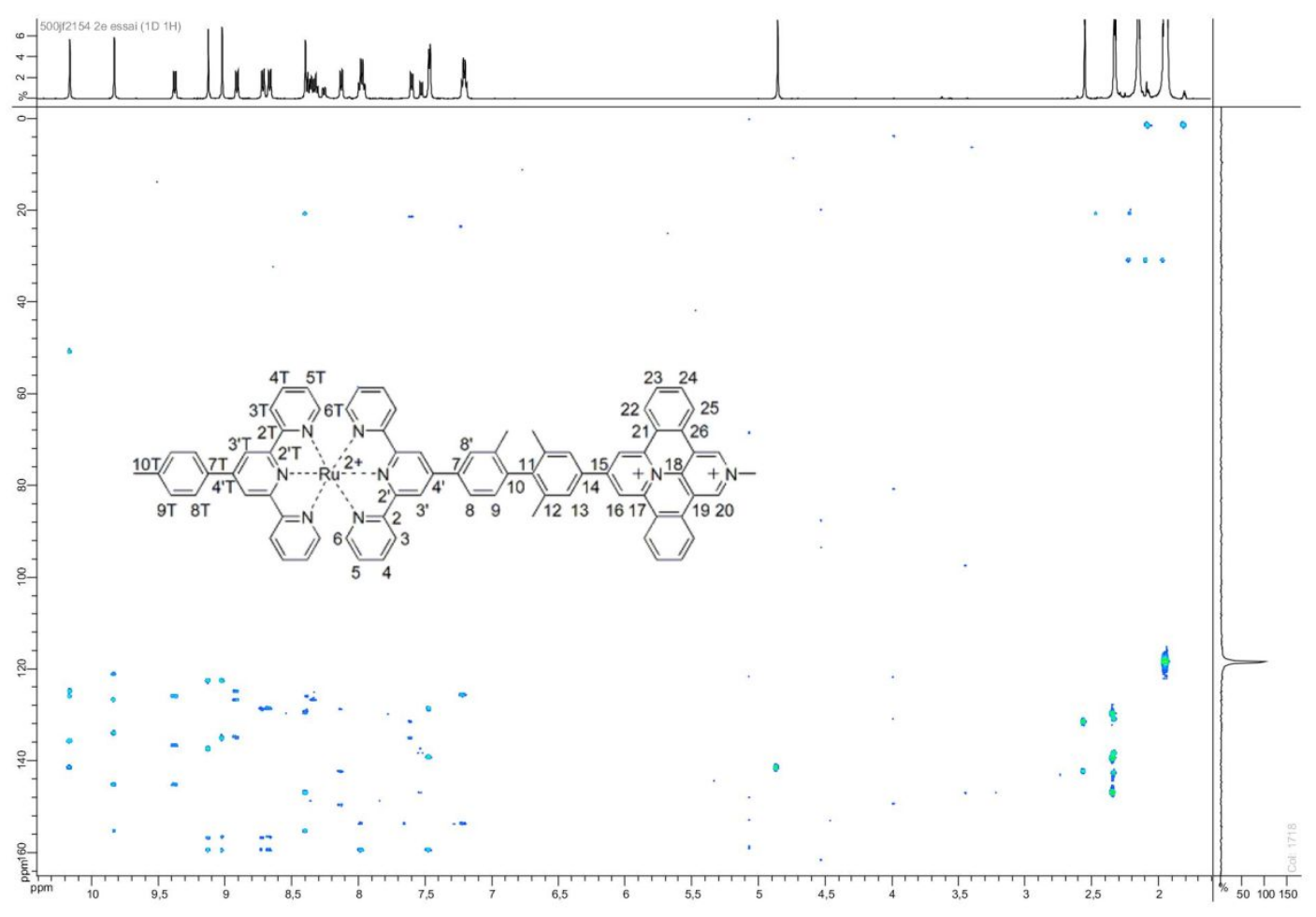

Figure S-21c. HMBC NMR spectrum (500 and $126 \mathrm{MHz}$ ) of 3 in $\mathrm{CD}_{3} \mathrm{CN}$ (full spectrum).

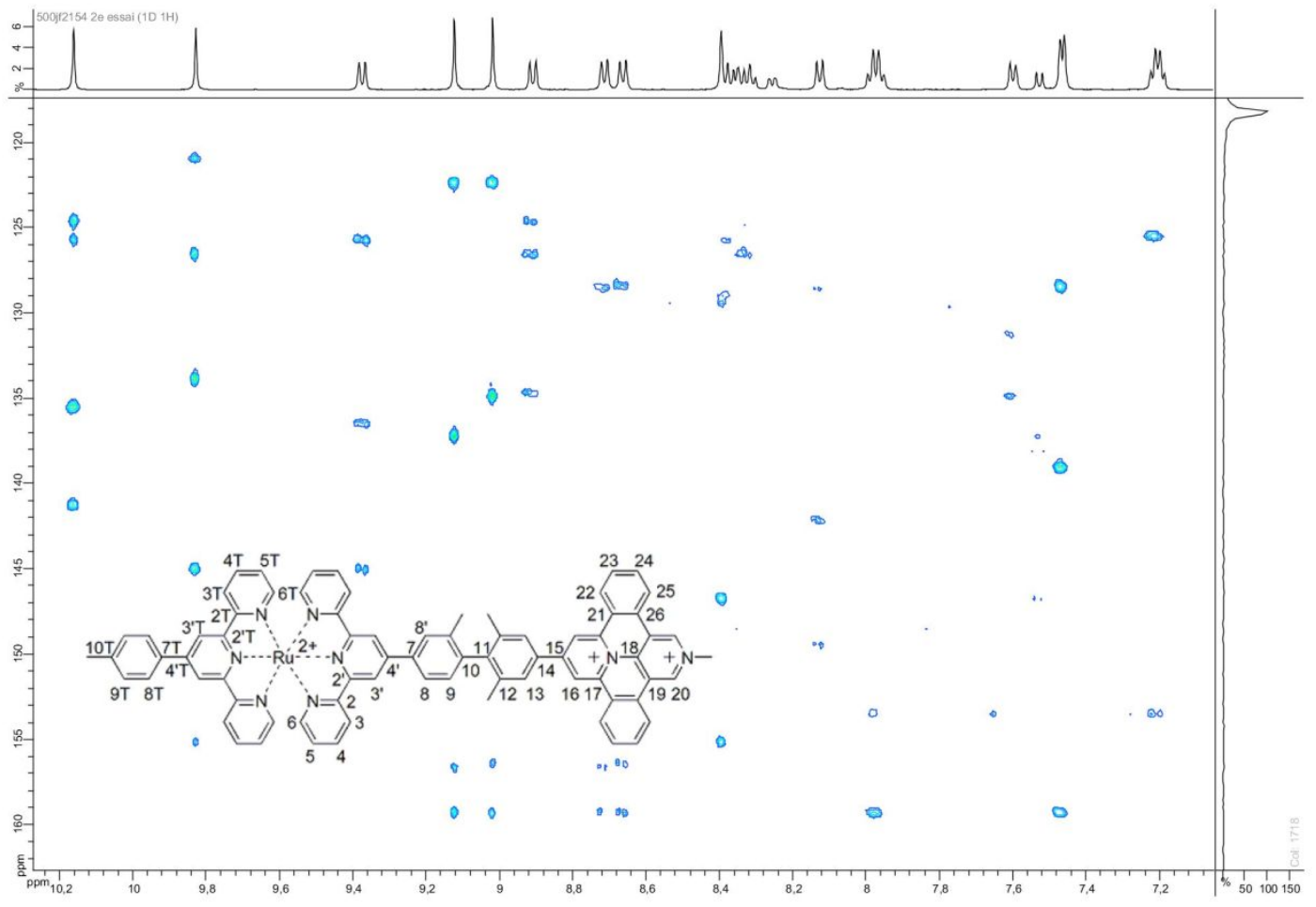

Figure S-21d. HMBC NMR spectrum (500 and $126 \mathrm{MHz}$ ) of 3 in $\mathrm{CD}_{3} \mathrm{CN}$ (aromatic part). 


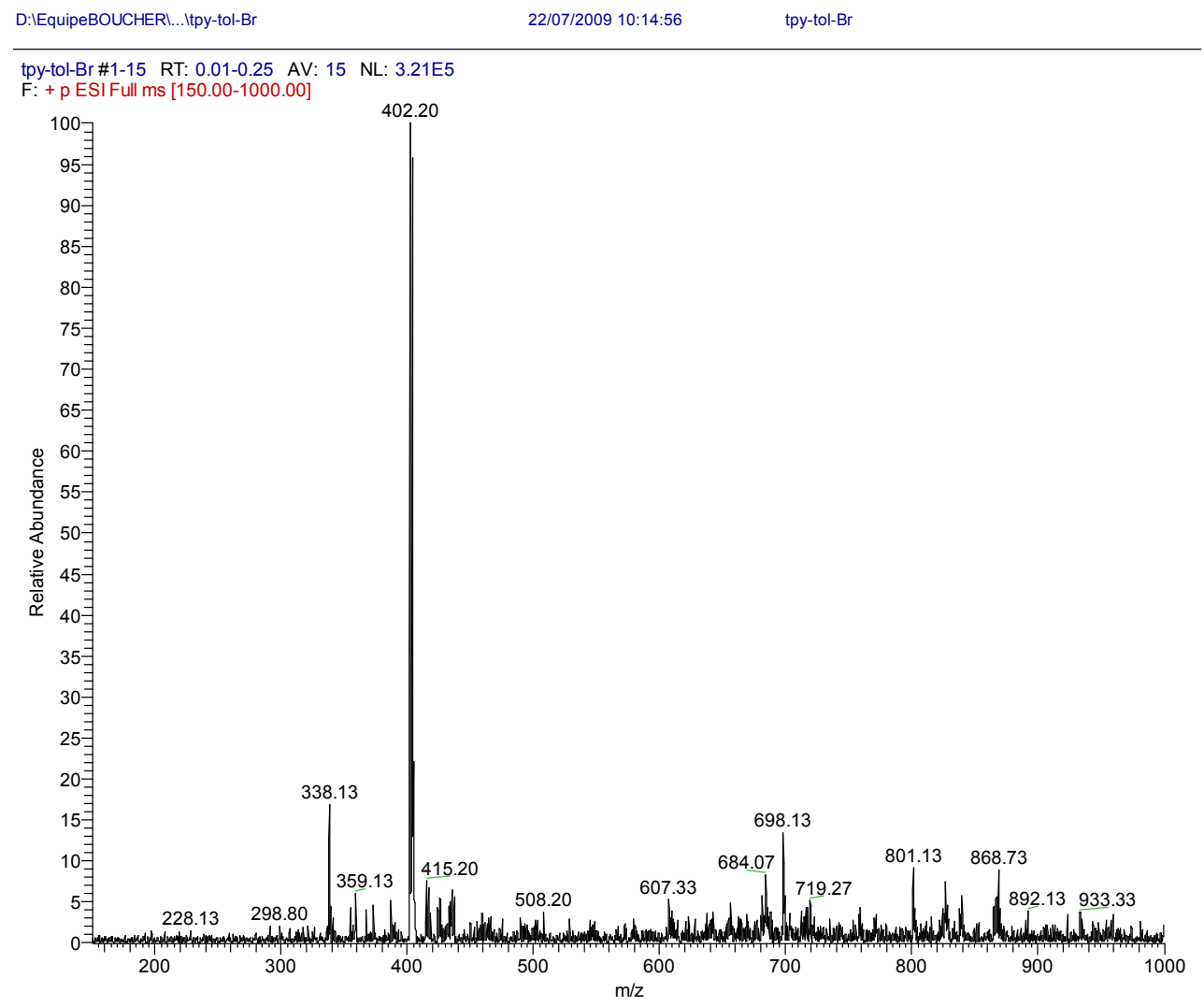

Figure S-22a. Mass spectrum of 4'-(4-bromo-3-methylphenyl)-2,2':6',2"-terpyridine (low resolution and full spectrum).

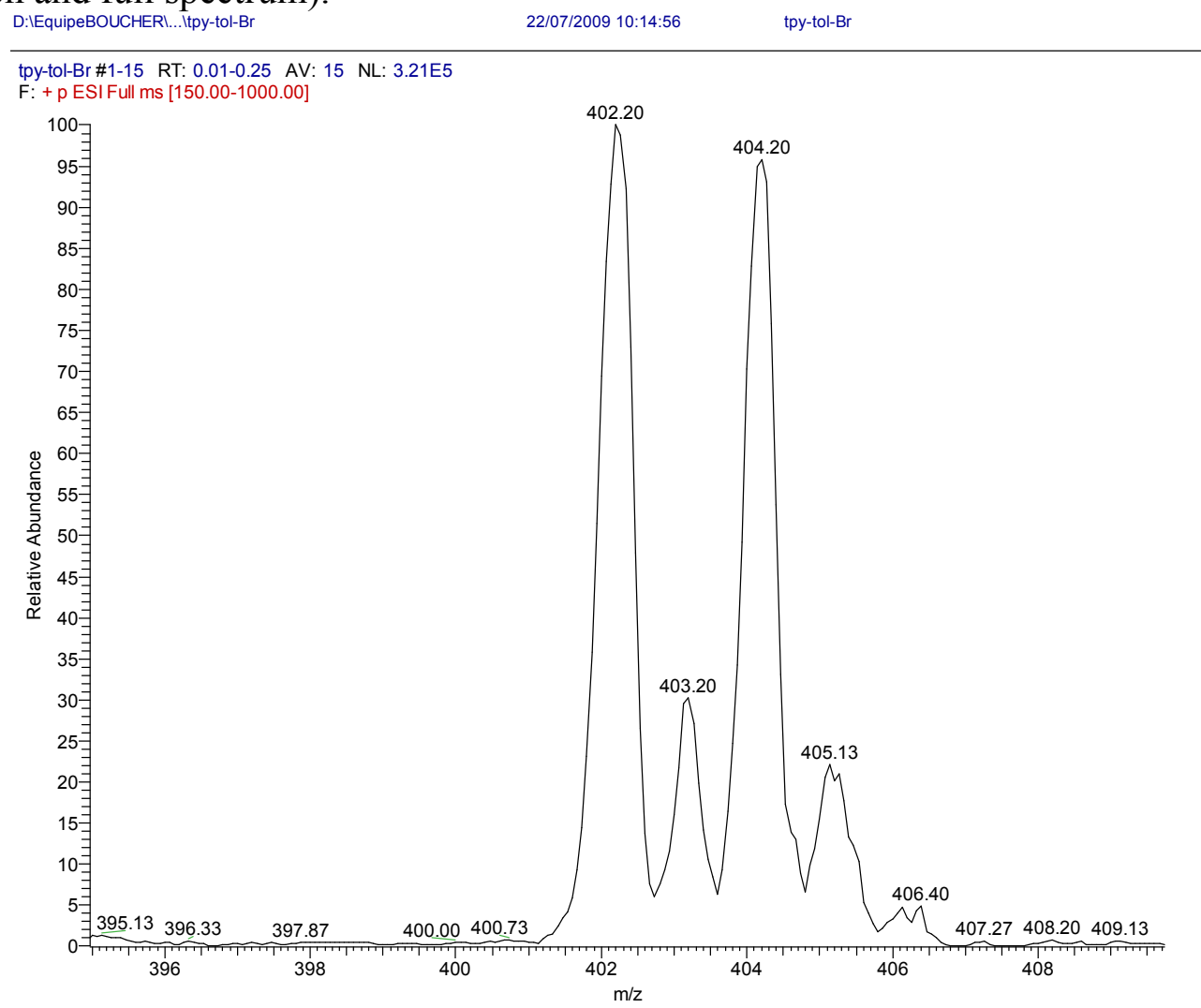

Figure S-22b. Mass spectrum of 4'-(4-bromo-3-methylphenyl)-2,2':6',2"-terpyridine (low resolution and zoom). 


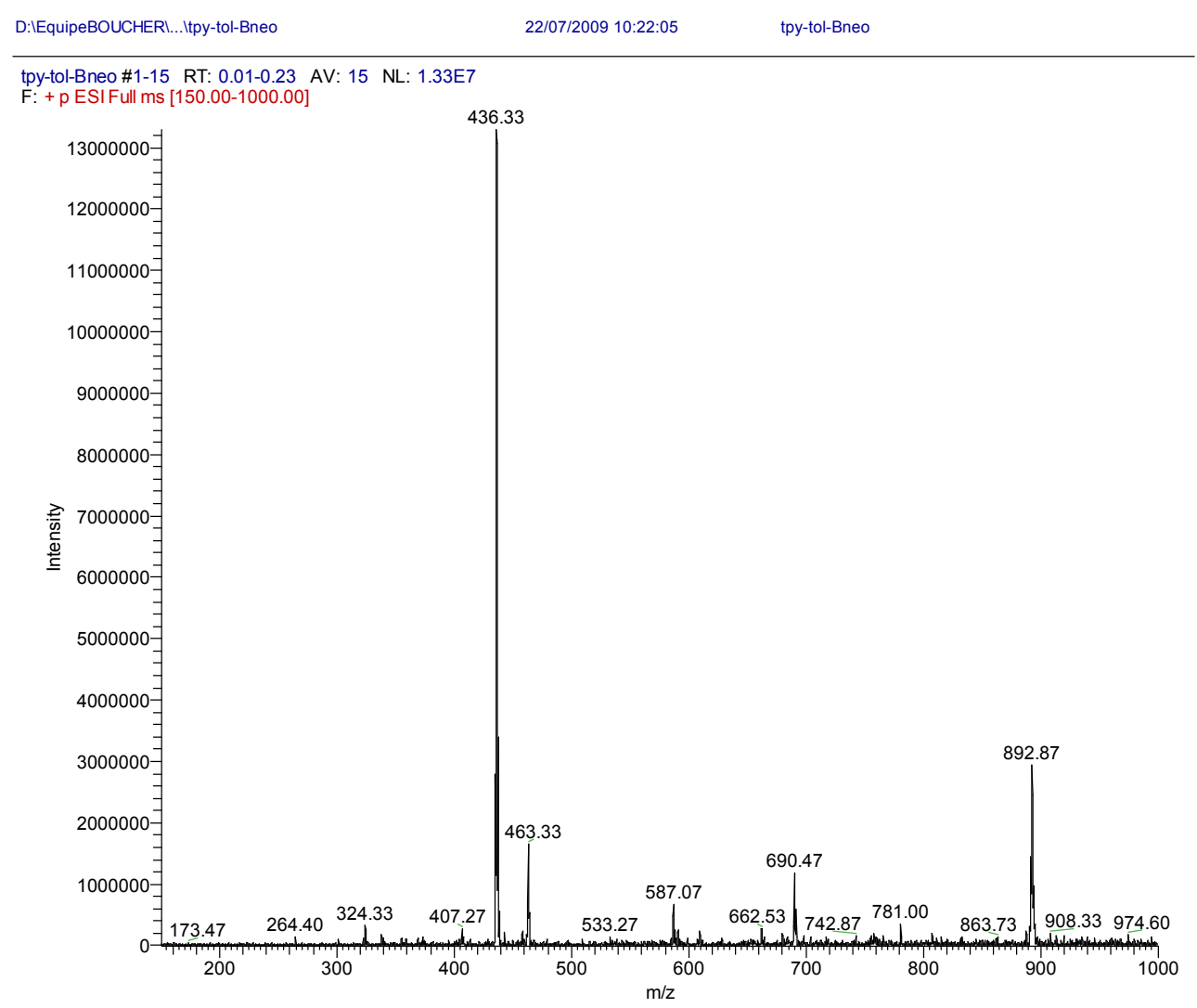

Figure S-23a. Mass spectrum of 4'-[3-methyl-4-(neopentylglycolatoboron)phenyl]-2,2':6',2"terpyridine (low resolution and full spectrum).

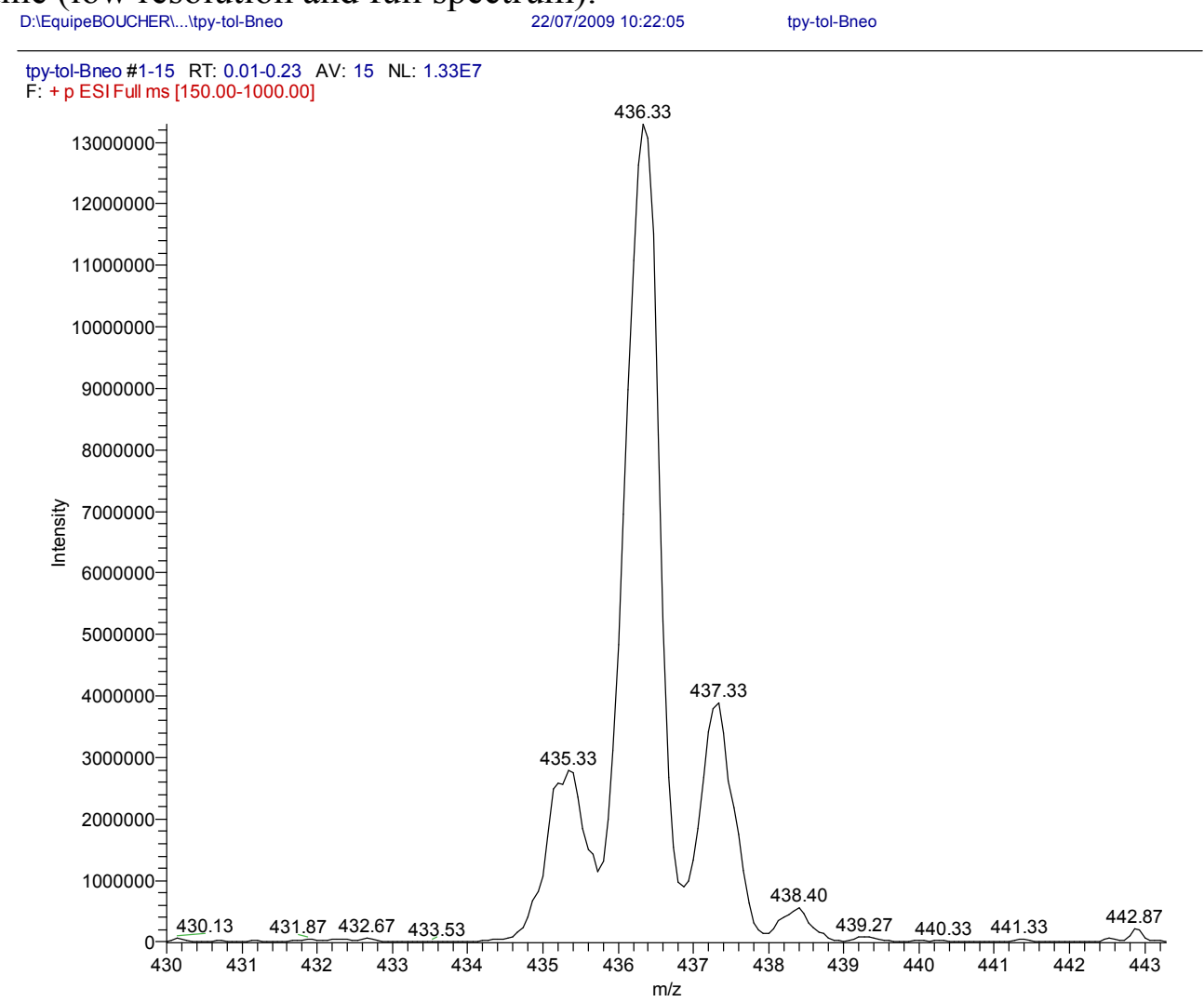

Figure S-23b. Mass spectrum of 4'-[3-methyl-4-(neopentylglycolatoboron)phenyl]-2,2':6',2"terpyridine (low resolution and zoom). 


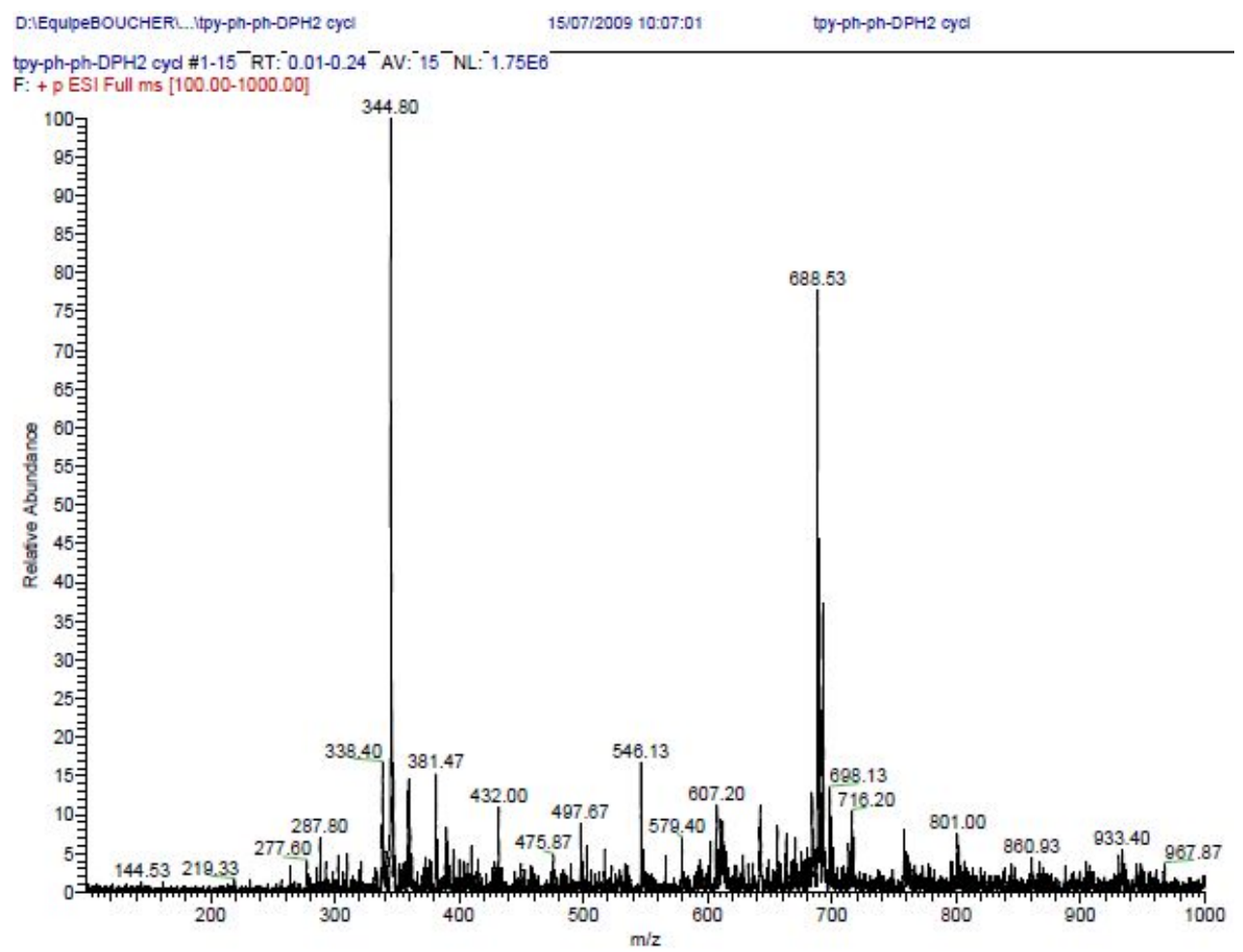

Figure S-24. Mass spectrum of ligand 1 (low resolution).

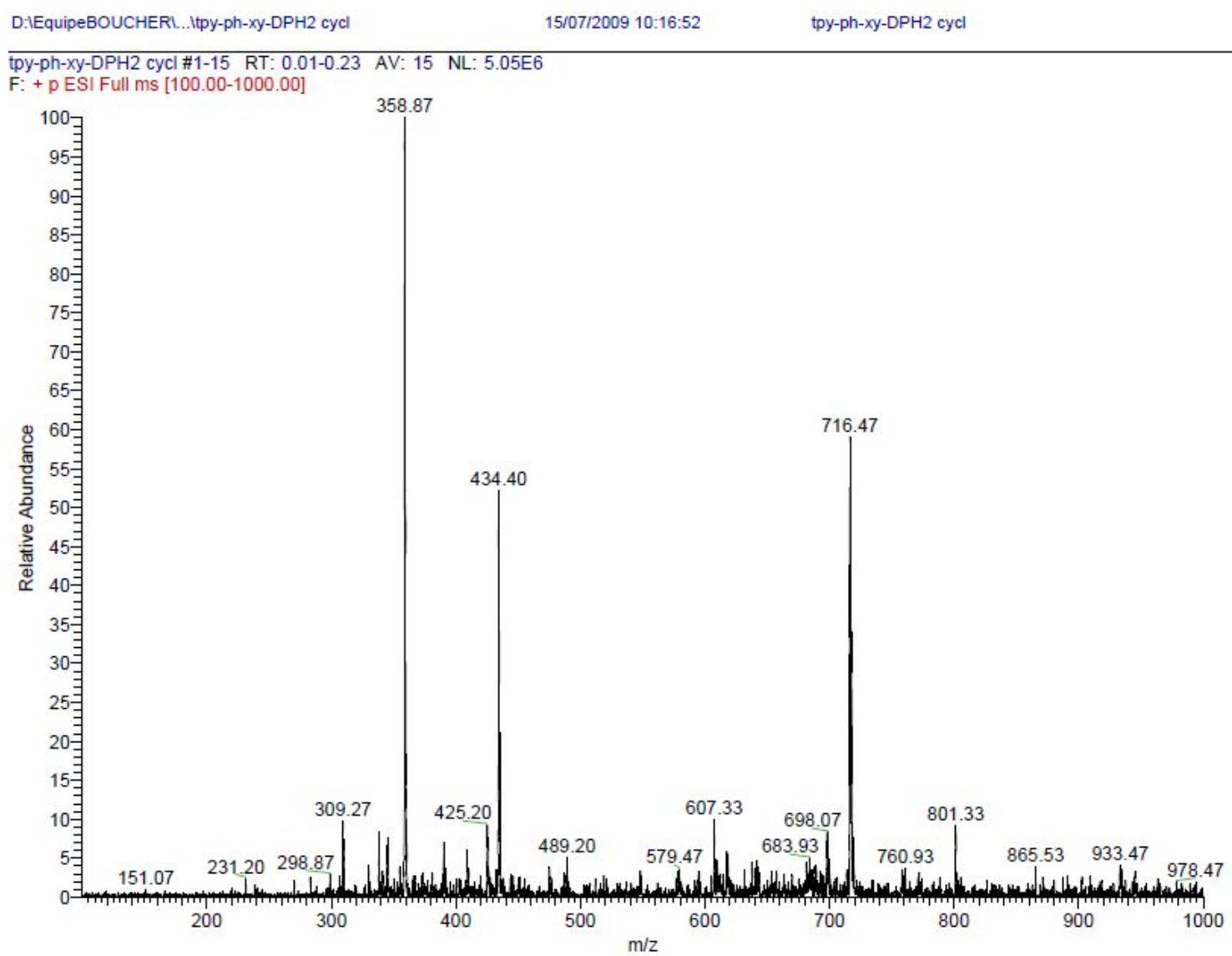

Figure S-25a. Mass spectrum of ligand 2 (low resolution and full spectrum). 
F: + pESI Full ms [10000-1000.00]

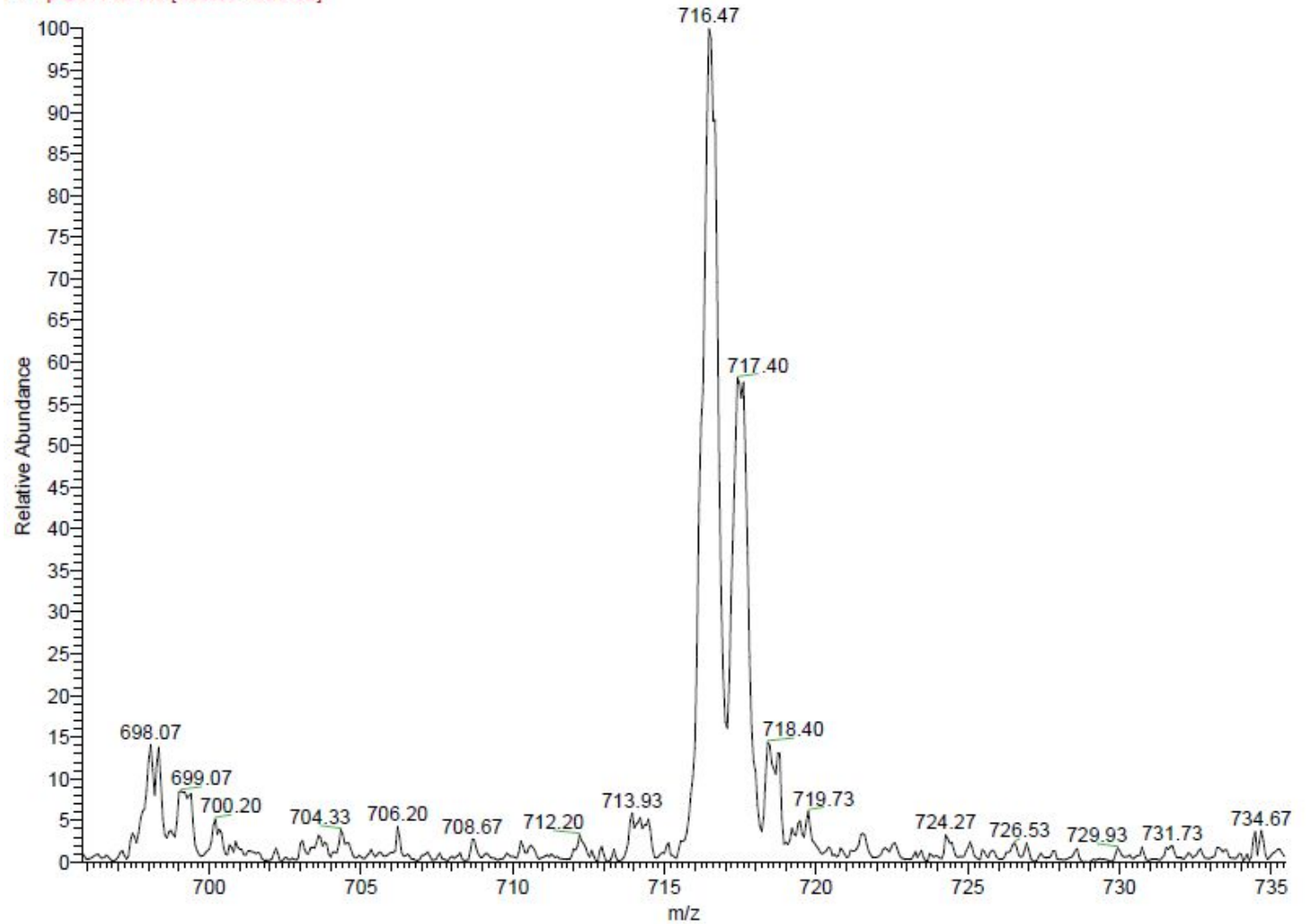

Figure S-25b. Mass spectrum of ligand 2 (low resolution and zoom).

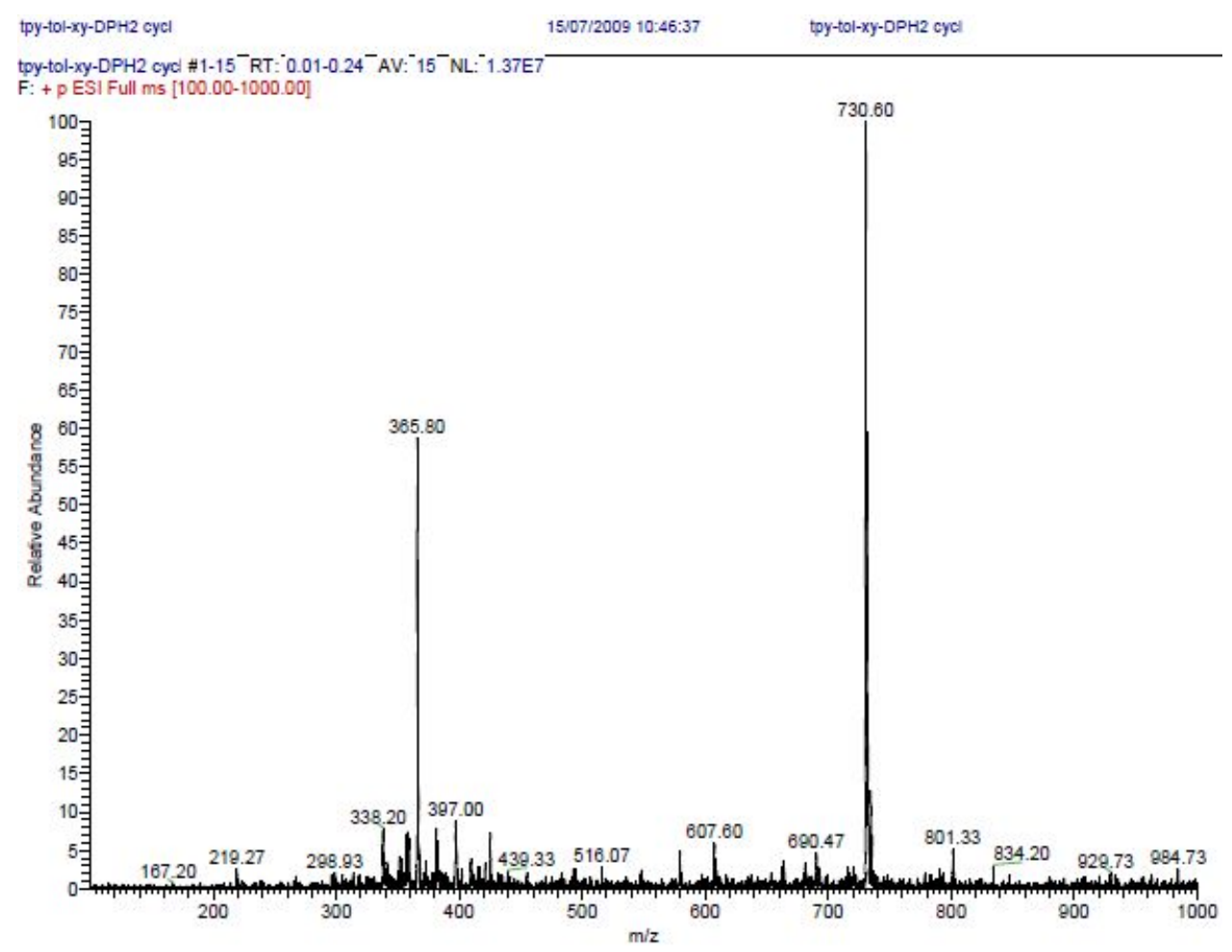

Figure S-26a. Mass spectrum of ligand 3 (low resolution and full spectrum). 
tpy-tol-xy-DPH2 cycl \#1-15 ${ }^{-} \mathrm{RT}^{-} \mathrm{C}^{0} 0.01-0.24^{-} \mathrm{AV}^{-}{ }^{-15}{ }^{-} \mathrm{NL:}{ }^{-} 1.37 \mathrm{E}$

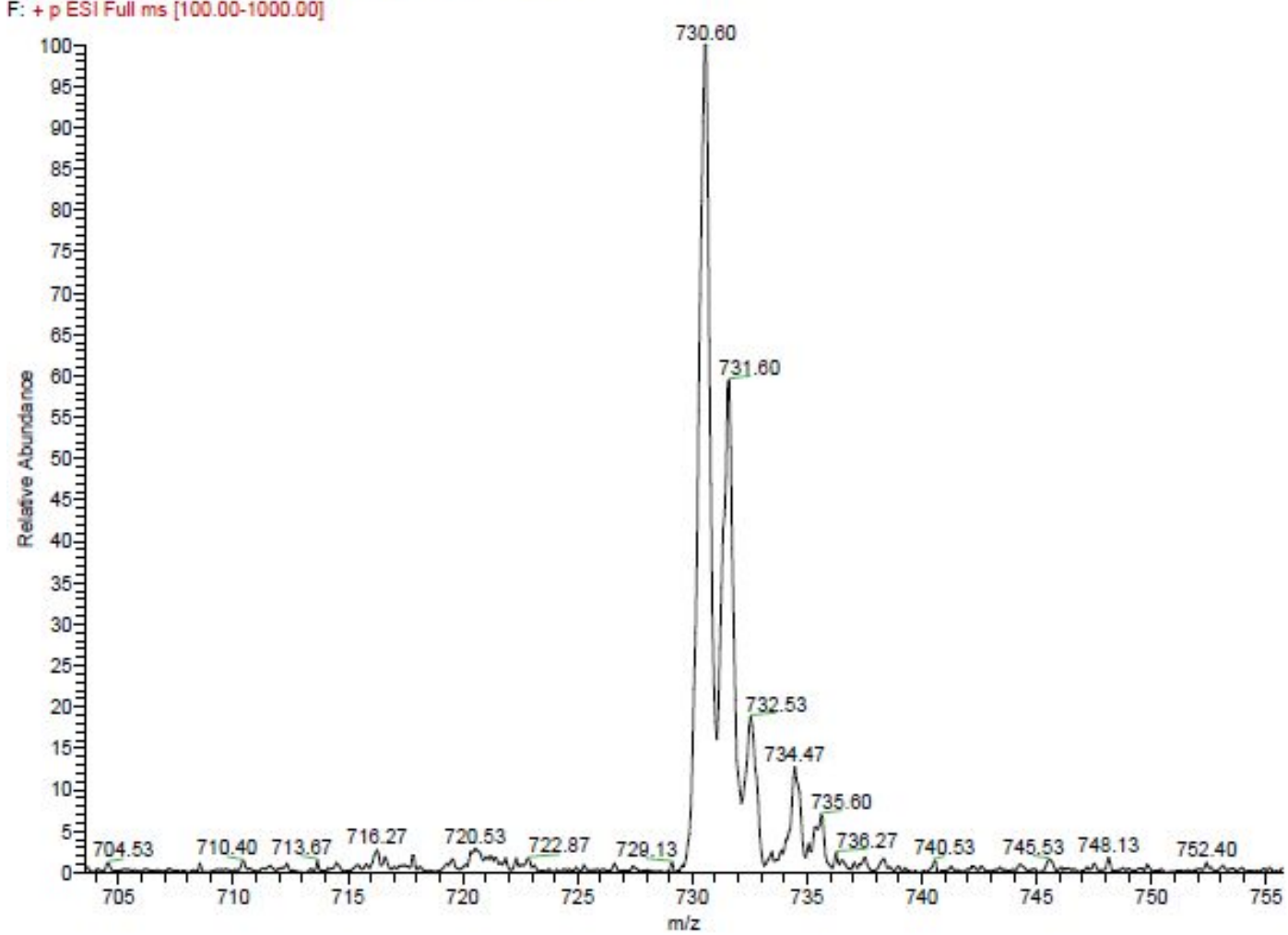

Figure S-26b. Mass spectrum of ligand 3 (low resolution and zoom).

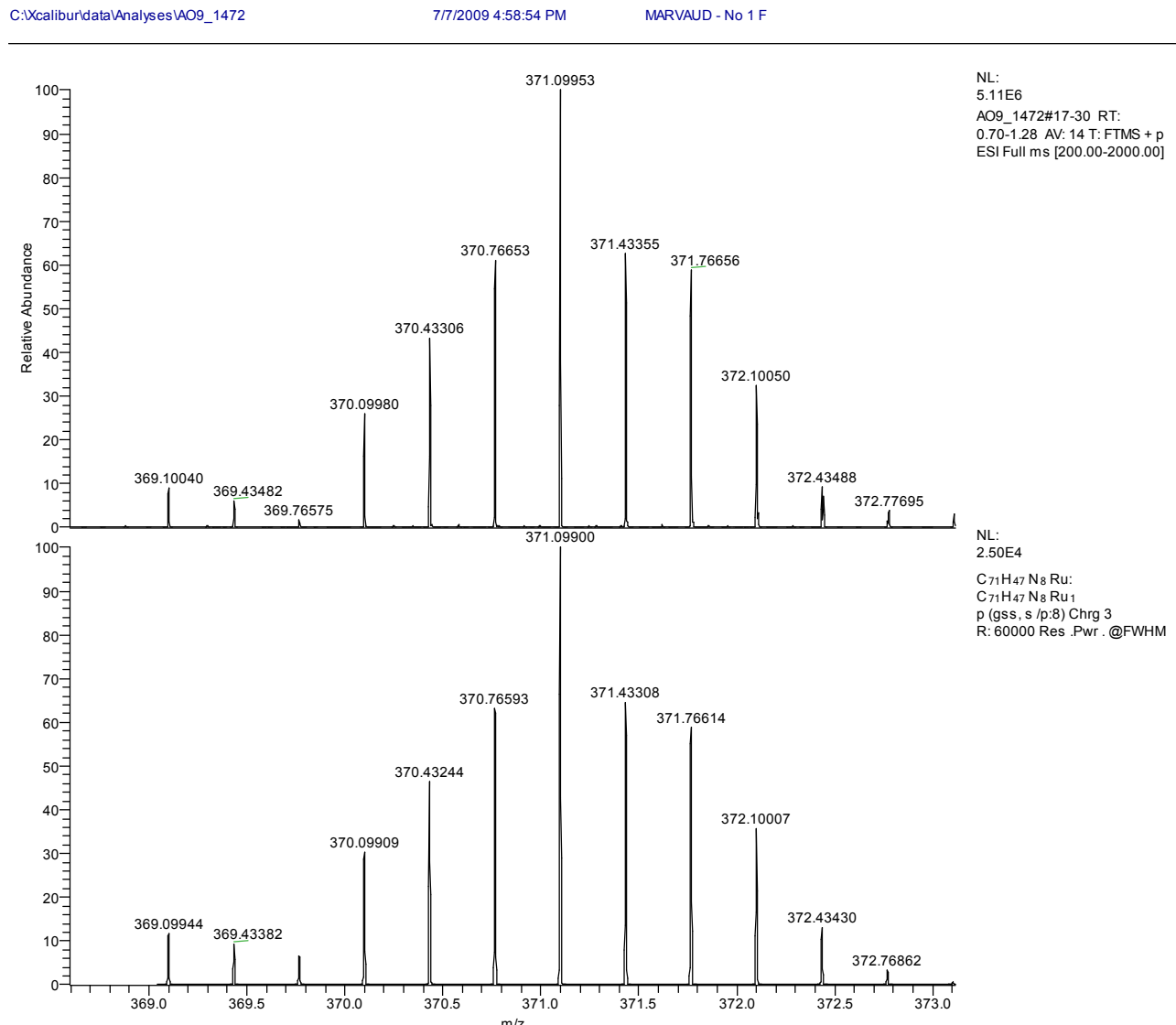

Figure S-27. Mass spectrum of dyad 1 (high resolution FTMS). 
Monoisotopic Mass, Odd Electron lons

243 formula(e) evaluated with 2 results within limits (all results (up to 1000) for each mass)

C: $1-100 \quad H: 0-110 \quad N: 0-12 \quad$ Ru: 0-1

13-Jun-2014 13:49:51
ITODYS_Dyad10-Ru-D 106 (0.586) Cm (99:123)

CH3CN

LCT Premier XE KE 483
1: TOF MS ES++

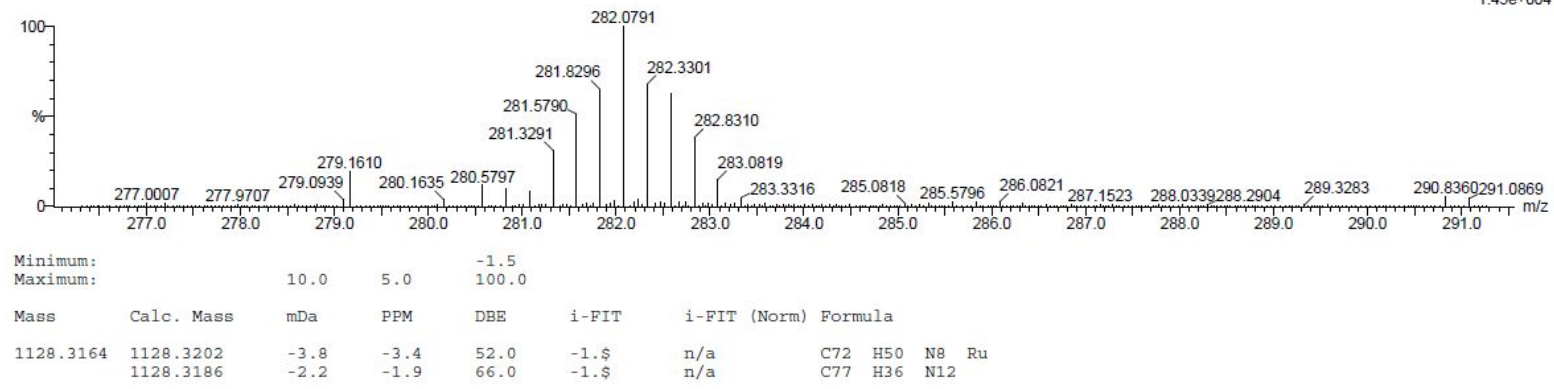

Figure S-28. Mass spectrum of 1 (high resolution FTMS).

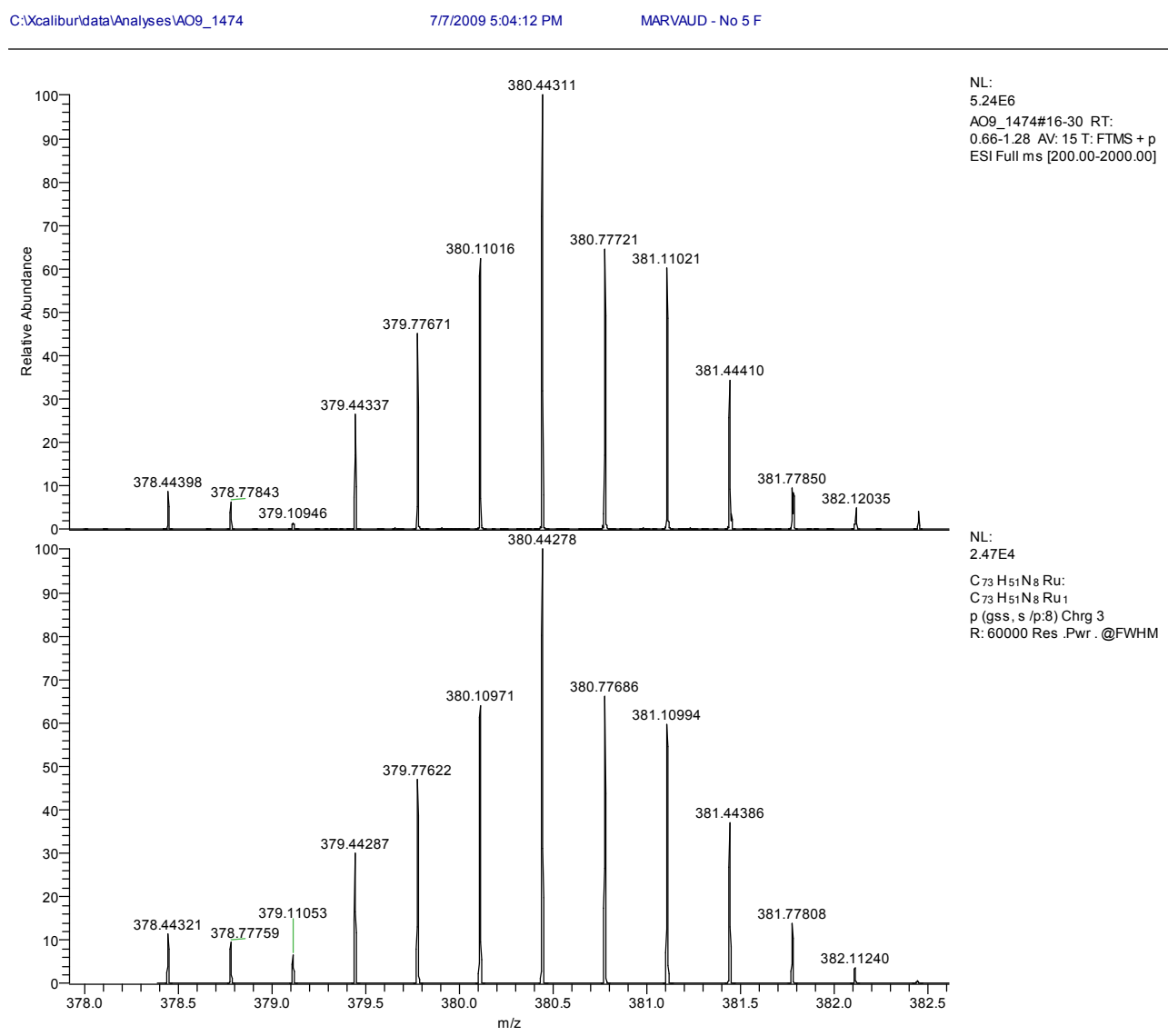

Figure S-29. Mass spectrum of dyad 2 (high resolution FTMS). 


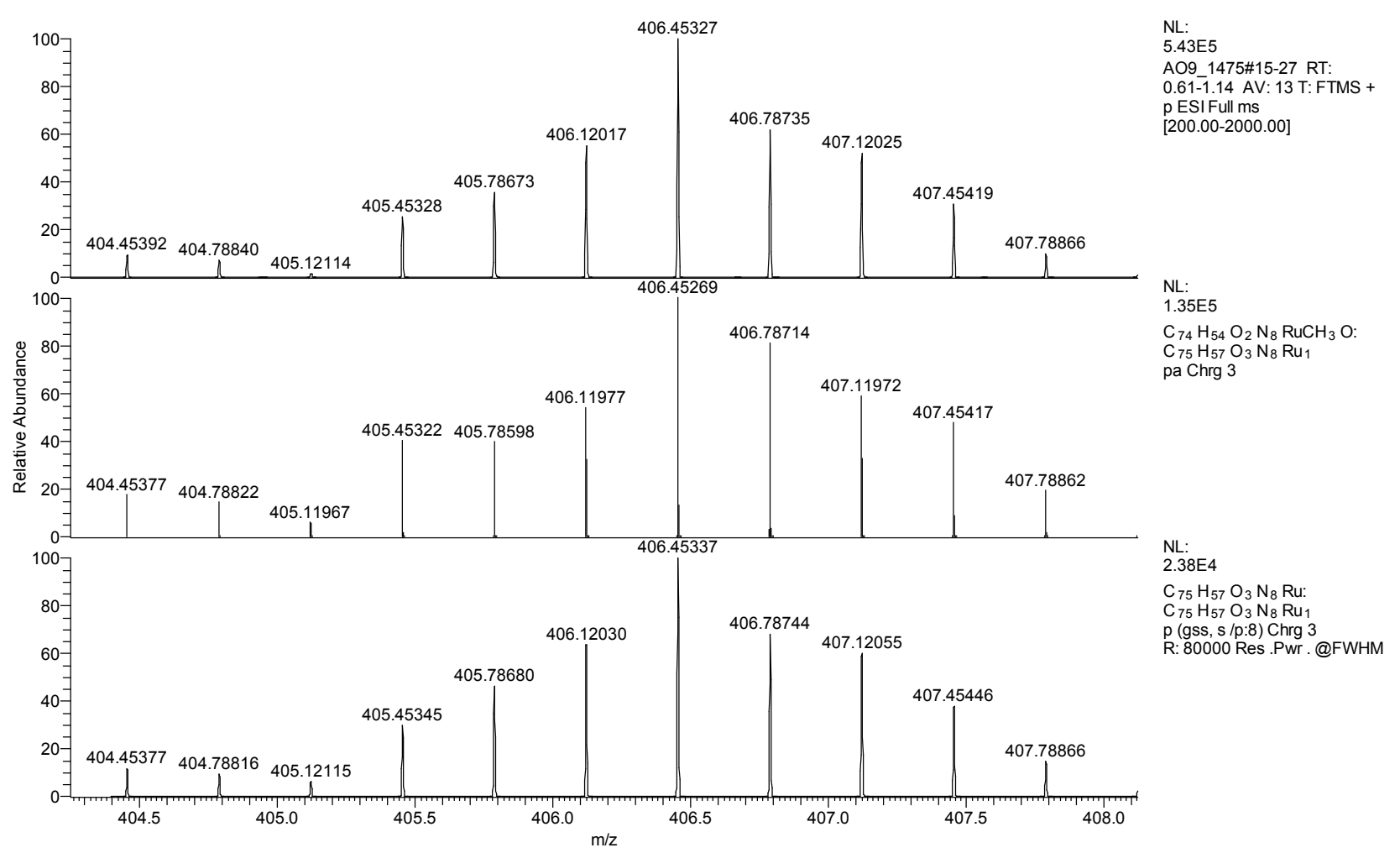

Figure S-30. Mass spectrum of 2 (high resolution FTMS).

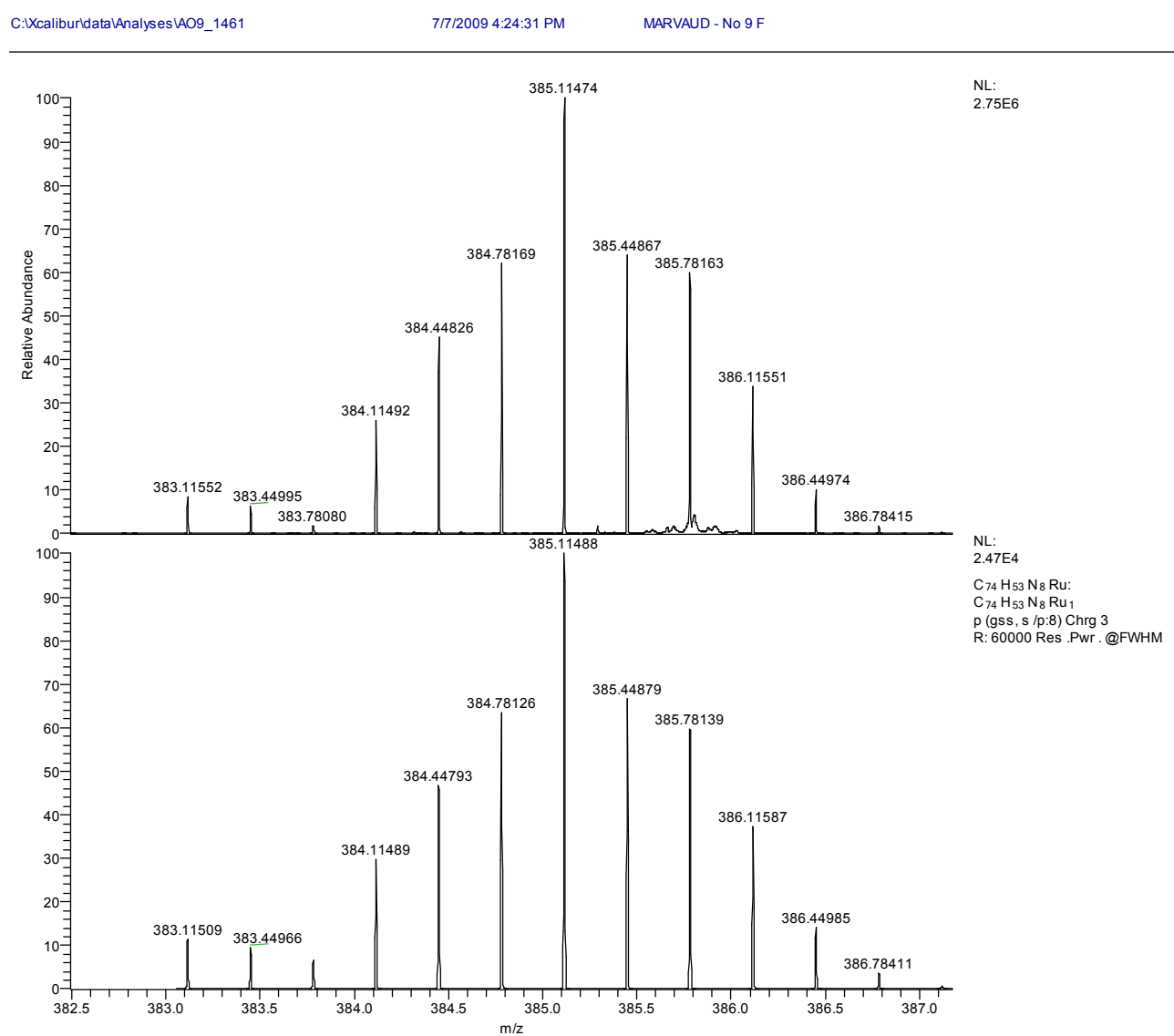

Figure S-31. Mass spectrum of dyad 3 (high resolution FTMS). 


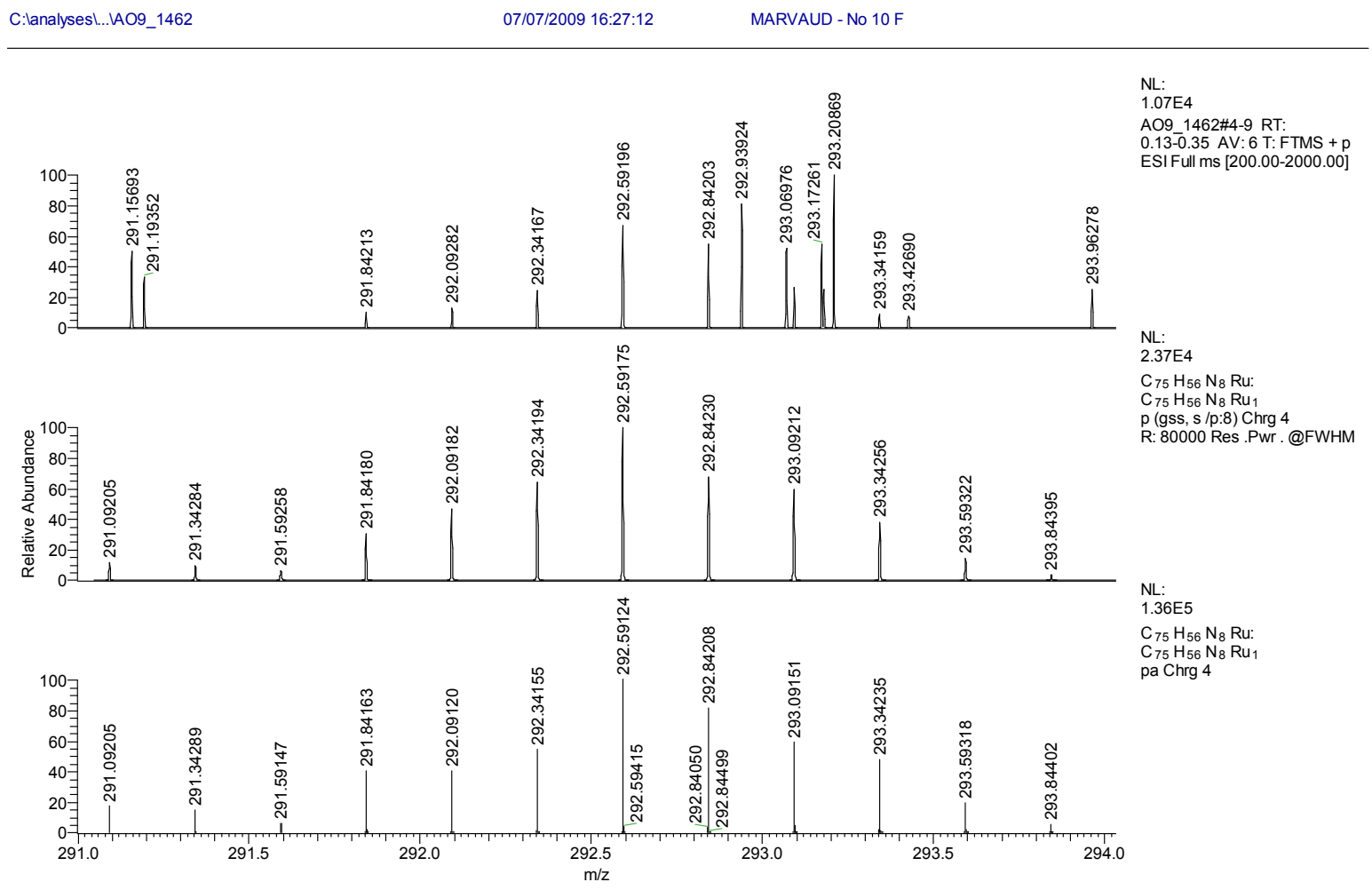

Figure S-32. Mass spectrum of $\mathbf{3}$ (high resolution FTMS). 